\title{
La chaîne opératoire a 70 ans : qu'en ont fait les préhistoriens français
}

\author{
Françoise Audouze, Claudine Karlin \\ UMR7041 Archéologies et Sciences de l’Antiquité. CNRS. ArScAn-Ethnologie Préhistorique, France. \\ Email: Audouze: francoise.audouze@mae.u-paris10.fr; Karlin: claudine.karlin@mae.u-paris10.fr
}

\section{Résumé :}

L'article porte sur l'origine et le développement de l'école française de technologie préhistorique. En remontant aux sources, les auteurs montrent comment la chaîne opératoire, concept proposé par A. Leroi-Gourhan, ethnologue et préhistorien, a d’abord été développé par les ethnologues puis appliqué à l'étude du lithique préhistorique en liaison avec eux.

La découverte du site de plein air de Pincevent a permis à Leroi-Gourhan de développer ses idées quant à une approche ethnographique d'une occupation préhistorique et les remontages de silex qui restituent les séquences de débitages ont été pour la première fois utilisées de façon systématique. La lecture de ces remontages a conduit à développer le concept de chaîne opératoire. Parallèlement $J$. Tixier concevait une méthode d'étude spécifiquement adaptée à l'analyse des productions taillées lithiques en s'appuyant sur la lecture de débitages expérimentaux pratiqués par son équipe. La mise en commun des idées développées par Tixier et son équipe et par le laboratoire d'Ethnologie préhistorique de Leroi-Gourhan, la pratique de l'expérimentation, de la taille du silex et des remontages de nucléus a conduit à des développements qui ont porté d'abord sur les techniques et méthodes de taille. Puis cette coopération a permis d'affiner une identification des niveaux de compétence, de l'apprentissage à la haute performance, puis, au delà, une reconnaissance des individus tailleurs à partir de caractéristiques idiosyncratiques de débitage. S'ouvrait ainsi le chemin vers l'analyse du domaine économique et enfin des domaines cognitif et social à partir de l'organisation spatiale d'habitats du Paléolithique supérieur.

Le concept et tous les champs de recherche qu'ouvrait son application ont ensuite été adoptés dans d'autres domaines que le lithique : l'os, la céramique, le métal, l'archéozoologie et le funéraire, impliquant le développement de critères appropriés et de techniques d'observation et d'expérimentation adaptées au matériau ou aux pratiques. Tout en s'appuyant sur les acquis, deux démarches récentes portant sur le moyen et long terme ouvrent de nouvelles pistes.

Flexibilité, vision dynamique des processus et connexion structurelle de la sphère technique à toutes les dimensions humaines font de la chaîne opératoire une efficace machine à penser les processus de production et leur place dans la société.

Mots-clés : chaîne opératoire; économie du débitage; économie de la matière première; expérimentation ; méthode ; remontage mental ; savoir-faire ; système technique ; technique

Published by the School of History, Classics and Archaeology, University of Edinburgh ISSN: 2055-0472. URL: http://journals.ed.ac.uk/lithicstudies/

This work is licensed under a Creative Commons Attribution 2.5 UK: Scotland License. 
«L'effort fait pour définir les mots est à quelque degré le témoin d'une réflexion sur ce à quoi ils donnent existence ».

A. Leroi-Gourhan (1972: 321)

L'occasion nous est donnée de faire le point sur la notion de chaîne opératoire. Elle a fait l'objet de nombreux écrits et nous l'utilisons aujourd'hui, comme le montre les articles de ce numéro de Journal of Lithic Studies, sans peut-être la charger tous de la même signification. Loin de nous l'idée de l'enfermer ici dans une définition stricte. Certaines qu'il s'agit d'un outil que les ethnologues et les préhistoriens ont largement enrichi, nous tenterons plutôt de montrer à quoi il nous a servi et nous sert toujours et comment nous l'avons fait évoluer. D'autres avant nous se sont livrés à cet exercice, M. Soressi et J.-M. Geneste par exemple (2011) ou G. Tostevin (2011a; 2011b) dans le numéro spécial de PaleoAnthropology consacré à l'épistémologie des méthodes d'analyse du lithique, chacun dans une optique qui lui était propre.

\section{Apparition du concept chez les ethnologues}

Le concept de chaîne opératoire s'est mis en place dans un contexte de développement de la technologie, étude raisonnée des techniques, courant original de l'anthropologie française (Bonte 1986; Lemonnier 1986; Haudricourt 1987; Schlanger \& Sinclair 1990); et au delà, dans un véritable milieu extérieur favorable qui se développe très largement après 1945 , où le mot "chaîne » est couramment employé dans l'industrie (travail à la chaîne, chaîne de production, chaîne de montage, etc.). Comme le note S. Desrosiers (1991), ce concept apparaît avec M. Mauss en 1947. M. Maget parle, en 1953, de " chaîne opératoire de fabrication » ou "d'opération » (1953). Il propose une méthode pour étudier les activités à différents niveaux en les découpant comme un film en scènes, en phases, enfin en gestes élémentaires ou « atome » d'action (1953).

A. Leroi-Gourhan, à l'époque professeur d'ethnologie, poursuit de son côté une réflexion sur le comportement technique lors de ses cours de Formation aux Recherches Ethnologiques (CFRE) entre 1952 et 1959 à l'Institut d'Ethnologie du Musée de l'Homme. On en trouve référence dans les cours polycopiés édités en interne par la FGEL -section Lettres de l'UNEFde 1955-56, 1957 et 1958-59; dans le polycopié de son cours de 1955-56, il décrit le comportement technique comme s'inscrivant dans « l'opération technique [par] une chaîne de gestes, la chaîne opératoire ». Il n’en donnera une définition concise qu'en 1964, mais l'envisage dans les années cinquante pour son apport à l'évolution cognitive de l'homme préhistorique : "suivre les gestes éclat par éclat [permettra de] reconstruire avec certitude une part importante de la structure mentale du fabricant” (Leroi-Gourhan 1952 in Schlanger 2004: 134). C’est pourquoi la définition de la chaîne opératoire prend place dans un chapitre du Geste et la parole sur le langage où il rapproche outils et symboles comme relevant du même équipement cérébral : "La technique est à la fois geste et outil, organisée en chaîne par une véritable syntaxe qui donne aux séries opératoires à la fois leur fixité et leur souplesse. La syntaxe opératoire est proposée par la mémoire et naît entre le cerveau et le milieu naturel » (Leroi-Gourhan 1964: 162-164). C'est aussi pourquoi il n'en reparle dans les chapitres suivants du Geste et la Parole que pour évoquer des chaînes opératoires élémentaires, machinales, réfléchies (Leroi-Gourhan 1965: 29), périodiques ou exceptionnelles (LeroiGourhan 1965: 32), et des chaînes opératoires destinées à combler les vides encore très larges dans le comportement de la machine (Leroi-Gourhan 1965: 59). "Pour Leroi-Gourhan qui la nomme, la chaîne opératoire est dans le prolongement de sa vision biologique » (Schlanger 
1991; 2004). On est loin de la méthode analytique sophistiquée que va devenir la chaîne opératoire au cours des années.

Après Maget et Leroi-Gourhan, cette approche du fait technique a été développée et précisée par deux équipes d'ethnologues formés aux côtés de ce dernier. Elles ont pour responsables $\mathrm{R}$. Cresswell et $\mathrm{H}$. Balfet, tous deux étudiants de la première heure, puis ses collaborateurs au CFRE (Centre de Formation à la Recherche en Ethnologie). Ils sont chercheurs au CNRS depuis 1955 pour le premier, et 1947 pour la seconde. Leurs recherches de terrain à l'étranger dans les années 1950-1960 et les thèses de doctorat d'état qui en résultent (Cresswell 1969; Balfet 1977) constituent des développements des enseignements reçus de Leroi-Gourhan. Tous deux y insistent sur la dimension sociale des pratiques techniques qu'ils étudient. Ce n'est qu'à la fin des années soixante, au moment où LeroiGourhan entre au Collège de France, qu'ils passent l'un et l'autre à l'université : Université de Paris V en 1969 pour Cresswell qui succède à Leroi-Gourhan pour l'enseignement de l'ethnologie, Université d'Aix-en-Provence pour Balfet en 1968. Ils commencent alors à enseigner, formant et agrégeant autour d'eux des étudiants et des chercheurs souvent anciens élèves de Leroi-Gourhan comme J.-P. Digard. Ils organisent des séminaires de recherche à Paris $\mathrm{V}$ et au Musée de l'Homme où sont menées des réflexions collectives sur la chaîne opératoire.

Mais les ethnologues sont dans l'ici, le maintenant ou le proche passé et non dans la longue durée comme Leroi-Gourhan. Le développement du concept n'est pas comme chez ce dernier dans la perspective évolutionniste, diachronique, mais dans la synchronie du terrain ethnologique. Ils se reconnaissent pourtant dans la définition donnée par ce dernier. La chaîne opératoire de concept devient peu à peu une méthode et un puissant outil analytique pour lesquels ces ethnologues développent à leur tour des concepts pour en expliciter le potentiel.

Creswell crée l'unité de recherche CNRS Techniques \& Culture. En 1976, dans l'introduction au premier numéro de ce qui deviendra la revue Techniques \& Culture, Creswell met la notion de « chaîne opératoire » au centre même de la recherche de son équipe (1976: 6). Il encourage l'expression de divers points de vue traduisant différents niveaux d'analyse. Cette revue publiera régulièrement des articles épistémologiques qui ont eu un rôle particulièrement stimulant dans notre communauté. On doit à Cresswell, puis aux chercheurs de son équipe d'avoir exploré les relations entre processus techniques et réseaux sociaux, avec notamment la notion de verrou social à laquelle peut se heurter l'innovation technique.

Dans le même temps Balfet succède à Leroi-Gourhan à la tête du département de Technologie comparée que ce dernier avait dirigé au Musée de l'Homme tout en étant détachée à l'Université d'Aix-en Provence pour y enseigner l'ethnologie des techniques. En 1975, dans le chapitre "Technologie » du manuel Éléments d'Ethnologie 2, dirigé par Creswell, Balfet parle du « découpage de la chaîne opératoire par séquences et opérations élémentaires jusqu'(...aux) atomes techniques ; ici nous intéresse au premier chef ce qui est considéré comme début et fin de la chaîne, les coupures (...) et aussi les raccords avec d'autres opérations ou des chaînes annexes » (Balfet 1975: 67). Quelques années plus tard, elle organise un colloque qui porte l'intitulé provocateur : Observer l'action technique : des chaînes opératoires, pourquoi faire ? (Balfet 1991). Des préhistoriens y participent (Karlin et al. 1991).

P. Lemonnier, étudiant de Cresswell appartenant à la génération suivante et proche de ces deux courants, définit la culture matérielle comme une production sociale (Lemonnier 1980; Creswell 1983). Il distingue dans la chaîne opératoire les opérations stratégiques, incontournables pour mener à bien le projet, et les opérations susceptibles de variations laissées au choix de l'opérateur qui renvoient au contexte social et culturel (Lemonnier 1992; Schlanger 2005). Il souligne encore que la chaîne opératoire s'inscrit dans le temps : les éléments constituants « se succèdent, se déroulent simultanément ou se chevauchent; si une 
chaîne opératoire possède un début et une fin, elle n'est pas nécessairement linéaire » (Lemonnier 1976: 106). Puis il précise une autre dimension déjà évoquée par Balfet : " une activité technique peut demander l'organisation relative et la combinaison de plusieurs chaînes opératoires ; cette organisation et son déroulement dans le temps forment le processus technique » (Lemonnier 1976: 107). Sa thèse en donnera la définition la plus opératoire qu’il applique à une étude des salines de Guérande (Lemonnier 1984). Ses idées se diffusent largement chez les préhistoriens lors de séminaires communs à l'Institut d'Art et d’Archéologie de l’Université de Paris I (1981-1983).

Enfin, dans cette même période, Cresswell et Lemonnier, au delà d'une définition plus affinée et opérationnelle de la chaîne opératoire, poursuivent l'idée d'une relation entre étapes techniques et enjeux sociaux. Pour eux la technologie se doit d'aborder les rapports entre la technique et le social : " For the ethnologist, and for the archaeologist and historian as well, technologies are - like myths, marriage prohibitions, or exchange systems - social productions in themselves » (Lemonnier 1992: 1-2). La technologie culturelle va être au centre de leurs préoccupations.

Il ressort du travail des ethnologues que le concept de chaîne opératoire est un outil polyvalent qui permet d'aborder l'ensemble du fait technique. À ce titre il doit être extrêmement souple, en fonction d'échelles d'observation, de temps ou d'espace, adaptées à la problématique du moment. Le travail sur les différents découpages possibles de la chaîne opératoire n'est rien d'autre que l'élaboration d'une grille de lecture sur laquelle un groupe d'observateurs se met d'accord à un moment donné. Mais, quelle que soit l'échelle d'observation utilisée, cet outil doit tendre à mettre en évidence la logique interne d'une activité en cherchant à ordonner les opérations selon un ordre déterminé voulu par les acteurs. À ce niveau, il s'agit de mettre en évidence la succession des opérations nécessaires pour permettre d'aboutir à l'objectif recherché, d'affiner les unités à étudier (séquence, opération, geste) jusqu'à l'«atome » pour rendre compte de la logique de l'activité technique analysée. On prend aussi en compte les contraintes et les possibles que déterminent la matière, l'énergie, l'écosystème dont le rôle sur le déroulement de la chaîne opératoire est prépondérant, ainsi que des paramètres humains ou sociétaux. L'objectif n'est pas de simplement décrire tous les moments de la mise en œuvre d'une activité, mais, à travers le déroulé de ce synopsis, passage nécessaire à sa compréhension, de rendre compte des processus techniques et d'aborder les systèmes techniques comme expression d'une société.

\section{L’appropriation du concept par les préhistoriens}

\subsection{L’approche du laboratoire d'Ethnologie préhistorique : Les remontages}

Comment les préhistoriens se sont ils approprié ce concept ? La taille du silex fut le premier terrain d'application privilégié de cette approche. En effet, les différentes étapes de réduction d'un bloc de silex sont caractérisées par des successions de produits qui gardent les stigmates identifiables des modalités de leur extraction. Mais il a fallu un contexte exceptionnel pour que les préhistoriens, dépassant la seule typologie, mesurent le potentiel de cet outil d'analyse.

La découverte du site de Pincevent, en 1964, donne l'occasion à Leroi-Gourhan de mettre en œuvre ce à quoi il réfléchissait depuis longtemps, une approche ethnographique des occupations préhistoriques, ce que lui permettait enfin ce gisement magdalénien de plein air. Dans cet objectif, entre autres outils d'étude, il fait immédiatement des remontages une méthode d'analyse à part entière qu'il décrit comme «l'analyse détaillée des rapports entre nucleus et produits qui en sont sortis [grâce au remontage de 36 nucleus par C. Karlin]... dont l'étude technologique paraîtra ultérieurement» (Leroi-Gourhan \& Brézillon 1966: 338) (Figure 1). Il fait des remontages une démarche systématique et non une expérience 
ponctuelle vue comme une prouesse spectaculaire, ce qui fut souvent le cas pour les remontages effectués depuis le début du XX siècle, ou encore conduits pour résoudre des problèmes ponctuels (voir Cahen in Van Noten et al. 1978: 59). Il existe une exception notable, celle de W.H. Holmes qui invente la « core reduction sequence » en 1894 à partir des déchets de fabrication de pointes bifaciales d'une carrière ; ses publications étaient tombées dans l'oubli aux Etats-Unis jusque dans les années 70 et n'ont pas traversé l'Atlantique (Shott 2003: 96).

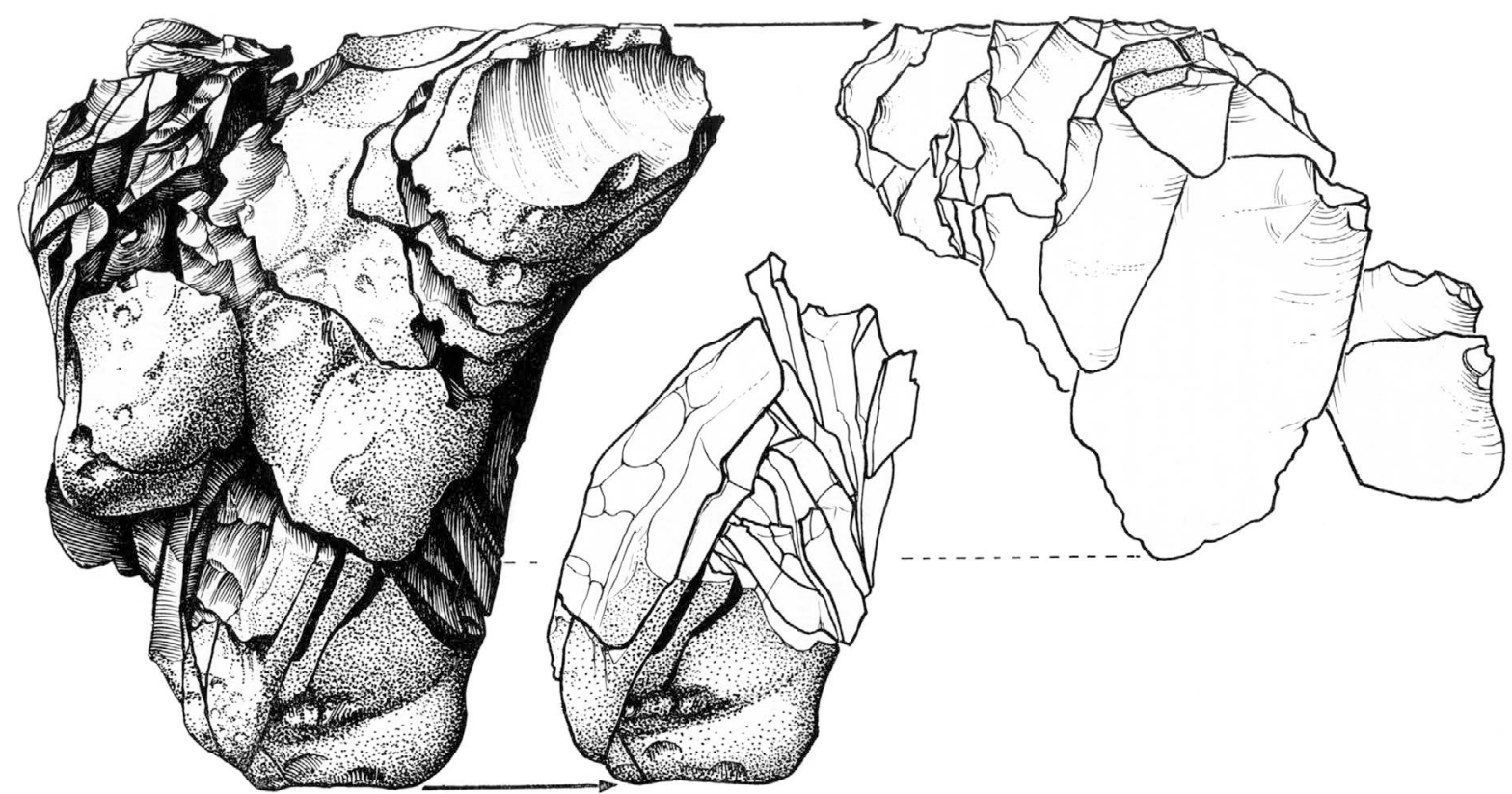

Figure 1. L'ensemble D22.240, un des premiers remontages réalisés lors de l'analyse de l’Habitation $n^{\circ} 1$ de Pincevent, est présenté dans la publication. On voit à gauche l'ensemble du remontage, à droite les éclats d'une phase de mise en forme vus par leur face d'éclatement, au centre le plein débitage avec les différentes réfections du plan de frappe (Leroi-Gourhan \& Brézillon 1966: 275, fig. 13, dessin de R. Humbert).

Figure 1. The D22.240 refit, one of the first refits realized for the analysis of Pincevent Habitation $n^{\circ} 1$, was published in the monograph. One can see the complete refit on the left, and on the right, the flakes coming from the shaping phase are seen from their removal surface. In the middle one can see the full laminar phase with all the rejuvenation flakes of the striking platform ((Leroi-Gourhan \& Brézillon 1966: 275, fig. 13, drawing by R. Humbert).

Mais Leroi-Gourhan, à ce moment-là, n’utilise ces remontages que pour nourrir une vision palethnographique des sols d'habitat dont il cherche à comprendre l'organisation. Cela apparaît tant dans la publication de l'Habitation n¹ (Leroi-Gourhan \& Brézillon 1966) que plus tard dans celle de la section 36 (Leroi-Gourhan \& Brézillon 1972), publications où la typologie tient une part importante alors que la technologie lithique est à peine balbutiante.

S’en suivent, après 1964, plusieurs années d'intenses remontages à Pincevent pendant lesquels émerge lentement la possibilité d'une approche technologique rendu possible par la richesse de la base de données peu à peu obtenue. Les relations suivies entretenues avec Balfet au sein du département de Technologie comparée qui abritait l'équipe de LeroiGourhan facilitent une appropriation progressive du concept de chaîne opératoire. En 1972, l'identification des composants des chaînes opératoires des blocs remontés est au point mais le regroupement en catégories, démarche héritée de la typologie (préparation, plan de frappe, entame, table d'enlèvement...), plutôt qu'en séquence d'opérations bloc par bloc, empêche pour un temps encore une lecture dynamique du processus de débitage (Karlin 1972: 263277). 
Il faut l'association de D. Cahen, assistant au Musée d'Afrique de Tervuren avec Karlin, membre de l'équipe Leroi-Gourhan, pour donner un véritable élan à une approche dynamique des remontages et signer un acte de naissance, même discret, au concept de chaîne opératoire comme outil d'analyse en préhistoire au service de la technologie lithique à partir des remontages (Cahen \& Karlin 1979; 1980). Réalisant de son côté des remontages sur un site congolais et sur le site de Meer en Belgique (van Noten et al. 1978), ce chercheur s'est inspiré des travaux de Karlin (Leroi-Gourhan \& Brézillon 1966: 276-279, 1972: 263-277) (Van Noten: 63, note 1) et a donné la première lecture dynamique des remontages à Meer (Cahen in Van Noten et al. 1978: 59 et 63). Cahen a été un activateur important dans le bouillonnement de réflexions autour de la technologie lithique que connut cette période (Cahen et al. 1979; Cahen \& collectif URA 28 1982).

La découverte d'autres gisements magdaléniens de plein air dans le Bassin parisien remet la chaîne opératoire et l'interprétation des remontages au centre des préoccupations de l'équipe d'Ethnologie préhistorique, et vient alimenter la réflexion technologique au sein de l'équipe : Les Tarterets 1 en 1970 où l'étude des nucléus « doit permettre une approche des techniques du débitage » (Karlin 1975), Etiolles en 1972 (Pigeot 1983), Marsangy en 19721974 (Schmider 1992) et Verberie en 1975-1976 (Audouze et al. 1981). Sur tous ces sites d'importantes campagnes de remontages des blocs de silex débités sont peu à peu effectuées.

Les premiers articles méthodologiques paraissent : la chaîne opératoire nous donne la capacité de restituer la durée, même s'il ne s'agit à ce moment-là que du temps bref d'une activité. «Chaque objet de pierre taillé est le produit d'une succession de gestes techniques orientés vers un but : fabriquer des outils destinés à satisfaire des besoins. La reconnaissance des chaînes opératoires permet à la fois de reconstituer les séquences de production et d'attribuer chaque pièce à l'une ou l'autre étape de la chaîne " (Cahen \& Karlin 1980: 12581268; voir aussi Cahen et al. 1980; Pigeot [Thèse 1983] 1987).

La méthode des remontages qui est à la base de l'analyse des chaînes opératoires a très vite fait des émules au delà de nos frontières. S'inspirant de l'expérience Leroi-Gourhan et dans la foulée des travaux réalisés à Pincevent qu'il suit avec attention, G. Bosinski promouvait chez lui les remontages : "The first attempt at applying lithic refitting to the investigation of a large prehistoric settlement site was made at Pincevent in France (LeroiGourhan \& Brézillon 1966; 1972). The results included increased knowledge of operational schemas, as well as of settlement organization and human behaviour. The successes of the Pincevent Project inspired researchers throughout North-West Europe to set up similar projects, usually focusing on the sites' latent spatial structures and human behaviour » (Ballin 2014 en ligne; pour le site de Rheindahlen: Bosinski 1966; Thieme 1983; 1990; pour le site de Gönnersdorf: Bosinski 1979). Mais la réflexion sur la technologie lithique reste bridée dans le contexte du site tandis qu'outils et débitage sont étudiés séparément.

En 1987 le « Big Puzzle Symposium », organisé par E. Cziesla, S. Eickhoff, N. Arts et D. Winter en Allemagne rassemble près de 50 chercheurs européens plus un américain et deux israéliens réalisant des remontages (Cziesla et al. 1990) : il apparaît que, si la méthode fait maintenant partie de nombreuses études de sites, la réflexion théorique autour de la notion de " chaîne opératoire " n'avance guère. Par ailleurs, l'association des remontages avec des études tracéologiques trouve un beau développement quelques années plus tard dans l'étude des sites magdaléniens comme par exemple Champreveyres et Monruz à Neuchâtel (Cattin 2002; 2012). 


\subsection{Une approche parallèle : L'expérimentation de la taille et le remontage mental de $J$. Tixier}

Parallèlement au lent mûrissement des idées en Ethnologie préhistorique et au musée de Tervuren, une autre approche de la chaîne opératoire s'est développée autour de J. Tixier, chercheur CNRS en poste à l'IPH depuis 1961, qui travaille sur la préhistoire du Maghreb. Lors d'un colloque fondateur aux Eyzies en 1964, ce tailleur expérimenté (Figure 2), rencontre un américain, Don Crabtree, fabuleux tailleur d'obsidienne et de silex. C'est au cours de ce colloque qu'apparaît pour la première fois l'expression " technologie lithique ». Tixier comprend que la voie expérimentale est un moyen efficace d'arriver à saisir les fondements techniques de la taille. Il est le premier à mettre à son programme la recherche des intentions des tailleurs. Dès 1967, il établit le rapprochement entre approche typologique et approche technologique: "Tendre à reconstituer dans leur intégrité et dans leur ordre successif les différents gestes techniques de l'ouvrier sculptant les différents stades morphologiques par lesquels une pièce est passée pour parvenir à sa morphologie définitive, et de là, décrire en connaissance de cause cette morphologie, telle est, fondée sur l'observation, la seule voie fondamentale nous permettant d'atteindre les intentions des artisans préhistoriques » (Tixier 1967: 773, in Perlès 1991b). Devenu chargé de cours à l'Université de Paris X en 1972, il crée un enseignement de technologique lithique en région parisienne, enseignement dans lequel la pratique expérimentale de la taille joue un rôle important. En 1978 il exprime son objectif de façon plus synthétique en se donnant pour but " la compréhension technologique de chaque pièce d'un ensemble industriel par la recherche des gestes successifs, donc des intentions de l'artisan qui l'a sculpté » (Tixier [polycopié 1978] 2012: 32).

Il crée le concept de méthode (agencement de différentes techniques) qu’il distingue de la technique (modalité du détachement de l'éclat, par exemple: percussion, pression, chauffe...) et travaille à une terminologie analytique rendant compte des stigmates caractéristiques des pièces liés à leur position dans l'enchaînement des gestes de taille (Tixier 1967; [polycopié 1978] 2012: 42). Sa méthode pour l'étude de l'outillage lithique, enseignée mais jamais publiée avant 2012, a une forte influence sur ses collaborateurs qui vont composer la future équipe de recherche CNRS Préhistoire et Technologie ainsi que sur ses étudiants.

Lors d'un colloque informel à Valbonne en 1979, Tixier réunit pour la première fois en France la nouvelle génération des spécialistes du lithique de tous bords : D. Binder, M.-L. Inizan, H. Roche, L. Meignen, C. Perlès issus de Paris X, mais aussi les Belges Cahen, M. Otte et F. L. Van Noten, M. Lenoir du laboratoire de F. Bordes et R. Desbrosses de l'IPH (1980). Il y exprime une gêne croissante devant la sécheresse de beaucoup d'études d'ensembles lithiques, ce qu'il appelle une «déshumanisation » de la pierre taillée. Il espère, avec ces chercheurs, trouver « d'autres voies, peu ou mal explorées, d'autres raisonnements [susceptibles de] nous faire mieux approcher un homme préhistorique bien souvent réduit à des chiffres et une étiquette » (Tixier 1980: 7). Il veut " promouvoir les études de technologie de la pierre taillée, c'est à dire la lecture exacte des gestes techniques sur une pièce, dans leur ordre chronologique » (Tixier 1980: 7), base pour une approche plus vivante de l'Homme. Il fait de l'expérimentation un socle de travail pour restituer le déroulement de l'acte technique qu'est la taille du silex ; de même il associe à sa démarche les raccord-remontages (Tixier 1980; Tixier et al. 1984).

C'est à l'occasion de réunions de travail du groupe de contact du FNRS à Tervuren et à Lièges, en 1979 et 1981 à l'invitation de Cahen (Cahen et URA 28 1982; Cahen 1984), que se fait la jonction entre des membres des équipes Préhistoire et Technologie de Tixier (IPH-Paris $\mathrm{X}$ ) et Ethnologie préhistorique de Leroi-Gourhan (Paris I), début d'une coopération toujours 
en cours. Dans ce groupe de réflexion, auquel se joint Karlin, est définitivement adopté le terme de "chaîne opératoire » utilisé par presque tous les intervenants au cours de leurs présentations (Cahen et URA 28 1982). En 1979 à Valbonne, il n’avait été utilisé dans la discussion que par Cahen, Otte et van Noten puis par Tixier et Inizan.

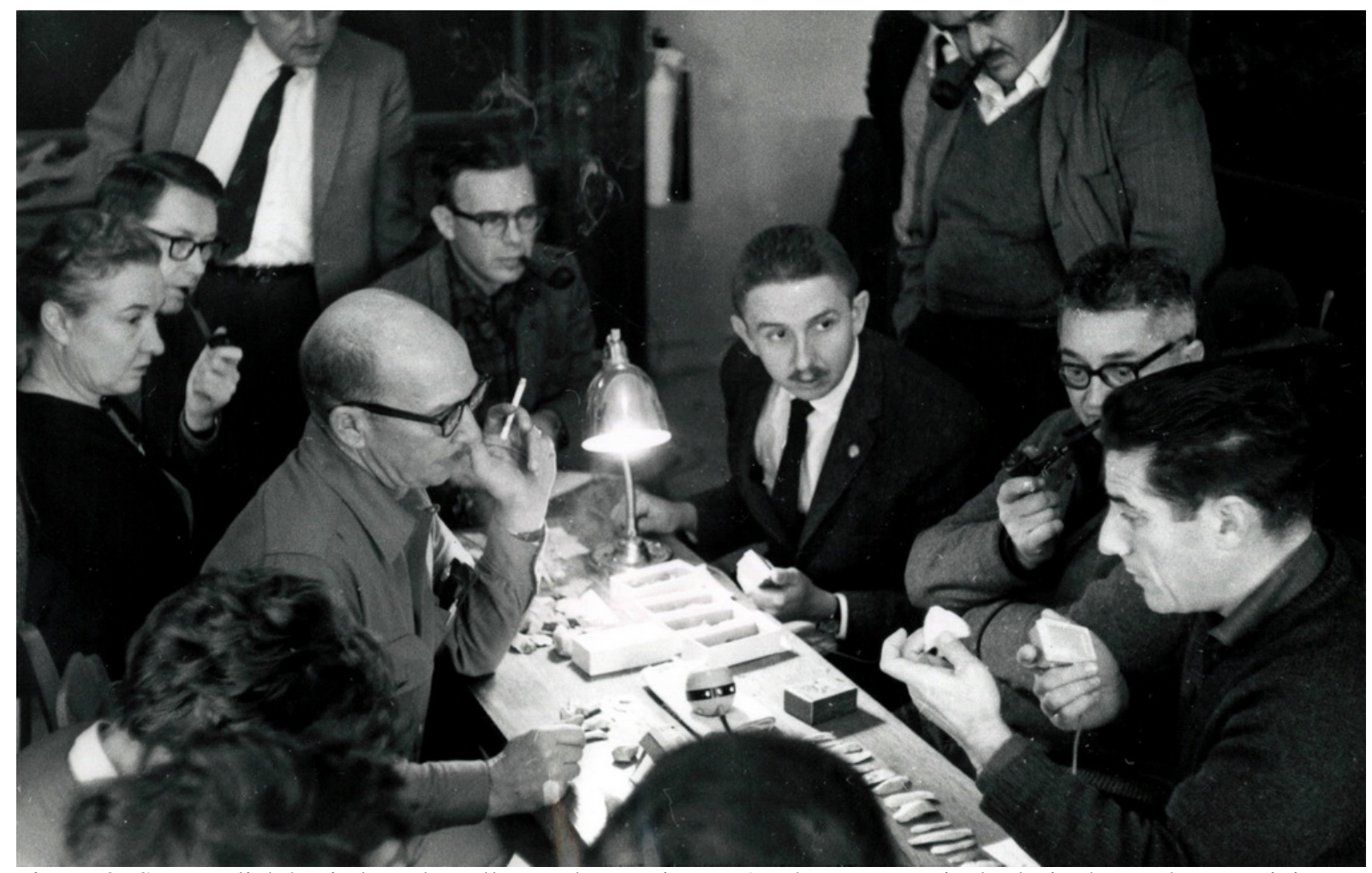

Figure 2. Sur ce cliché pris lors du colloque des Eyzies en 1964 on peut voir de droite à gauche : J. Tixier, F. Bordes avec sa pipe et A. Jelinek; en face, D. Crabtree et sa cigarette. Collection J. Tixier. (Tixier [polycopié 1978] 2012: 6, archives de J. Tixier, avec l'aimable autorisation des éditeurs).

Figure 2. Picture taken during the 1964 workshop at les Eyzies. From right to left: J. Tixier, F. Bordes with is pipe and A. Jelinek. Across the table D. Crabtree with a cigarette (Tixier [unpublished text 1978] 2012: 6, archives of J. Tixier, by permission of the publishers).

La réflexion croisée de tous ces chercheurs au cours de ces multiples réunions, notamment celles du Groupe de Contact du FNRS belge (Notae Praehistoricae 1981, 1983, 1985), va se développer et aboutir à un dépassement du concept brut en s'enrichissant des apports des uns et des autres, coopérations qui s'expriment dans des publications communes (Pelegrin et al. 1988; Pelegrin \& Karlin 1988; Ploux \& Karlin 1994, etc.). C’est ainsi qu'au sein de l'équipe Préhistoire et Technologie, les manuels de technologie lithique qui font connaître internationalement Tixier ont connu des versions successives : Tixier et al. 1980 t. 1 et 1984 t. 2; Inizan et al. 1992 t. 3 et 1995 t. 4, suivis d’une édition en anglais (1992 t. 3; 1999 t. 5) et d'une en japonais (1998 t. 3). Le tome 4 est désormais en ligne.

Au sein de l'équipe de Technologie Lithique (Préhistoire et Technologie, UMR 7050 CNRS-Université Paris X), durant les années 80, Inizan introduit le concept d'économie de débitage (Inizan 1976; 1980) et Perlès celui d'économie de la matière première (Perlès 1981; 1991b) qui conduisent plus largement à intégrer l'économie dans les problématiques liées au lithique. J. Pelegrin et E. Boëda poussent la réflexion sur les protocoles d'expérimentation et l'identification des caractéristiques diagnostiques (Pelegrin 2016). Pélegrin introduit une dimension psychologique en étudiant les relations entre capacités psycho-motrices et cognition (1985). Le concept d'économie du débitage est repris par N. Pigeot qui développe les notions de productivité et de niveaux de compétence au sein de l'équipe d’Ethnologie 
Préhistorique et dans son enseignement à l’Université de Paris I (Pigeot [Thèse 1983] 1987; 1986). La jonction entre les deux équipes va encore plus loin à partir de 1984 avec l'arrivée à Pincevent d'une étudiante en thèse de Tixier, S. Ploux qui travaille sur la caractérisation individuelle des tailleurs. S'ensuit une longue collaboration avec Karlin qui renforce les liens entre les deux équipes en mettant les deux écoles en synergie.

C'est aussi à la fin des années 70 que s'est opérée une jonction avec l'équipe de Techniques \& Culture, au cours de séminaires, de rencontres informelles à la Maison des Sciences de l'Homme à Paris, mais aussi dans un travail commun des élus de la section $33 \mathrm{du}$ Comité National du CNRS qui rassemble alors des anthropologues, des ethnologues et des préhistoriens. Cela génère une fertilisation croisée dont ces derniers vont tirer bénéfice (Karlin 1992). Des innovations se succèdent qui pour les unes relèvent du développement de la méthode initiale, pour d’autres représentent des dépassements théoriques et méthodologiques.

\section{La chaîne opératoire comme outil analytique : Exemple de la taille du silex au Magdalénien dans le Bassin parisien (France)}

Les remontages permettent de suivre les différents moments de l'exploitation d'un bloc décrite comme une succession de phases, d'opérations, de gestes. Le travail fait pour identifier chaque étape, chaque élément de la chaîne opératoire conduit à les nommer. Et, pour reprendre l'image de Balfet, plus petit est « l'atome » identifié plus riche est la reconstitution des enchaînements car plus nombreux sont les éléments pouvant apporter des informations. Mais aussi plus facile sera l'identification de cet "atome " lorsqu'il manquera dans une reconstitution de bloc travaillé. À l'inverse, certaines étapes produisant des objets caractéristiques ou portants des stigmates caractéristiques identifiés lors d'analyses de chaînes opératoires reconstituées, ces objets peuvent être reconnus et positionnés à leur juste place alors même qu'ils ne sont pas matériellement introduits dans leur propre chaîne opératoire de production. C’est le remontage mental, notion qu'a précisée Pelegrin ([thèse 1986] 1995b).

Les remontages devenus systématiques dans l'analyse d'une occupation préhistorique, l'identification de similitudes ou de régularités entre les chaînes opératoires lues sur différents blocs remontés et dont la contemporanéité est confirmée, conduit à reconstituer une chaîne modèle, le schème (Karlin et al. 1991). Il s'agit d'une entité abstraite, l'image d'une chaîne opératoire idéale permettant d'exploiter un bloc pour arriver à réaliser un objectif (Figure 3). Cette image partagée par tous les membres du groupe relève de leur patrimoine. C'est à ce schème que font référence Boëda, Meignen et J.M. Geneste lorsqu'ils dressent un inventaire des chaînes opératoires identifiées depuis le Paléolithique inférieur jusqu'au Paléolithique moyen, chacune répondant à des objectifs différents (Boëda et al. 1990: 44).

Ce schème renvoie à un savoir partagé par une collectivité : pour tel résultat on fait comme ça. Tout le monde sait comment il faut faire, ce qui ne veut pas dire que tout le monde sait faire, ni même tente de faire (Chamoux 1978). Mais « une histoire exemplaire ne peut être construite qu'en forçant dans son moule des épisodes empruntés à diverses histoires de vie qui constituent, quant à elles, autant d'exceptions à ce qui est censé être la norme commune » (Delbos \& Jorion 1987: 35). Il fallait donc passer de ce tailleur exemplaire aux individus auteurs de ces séquences. 

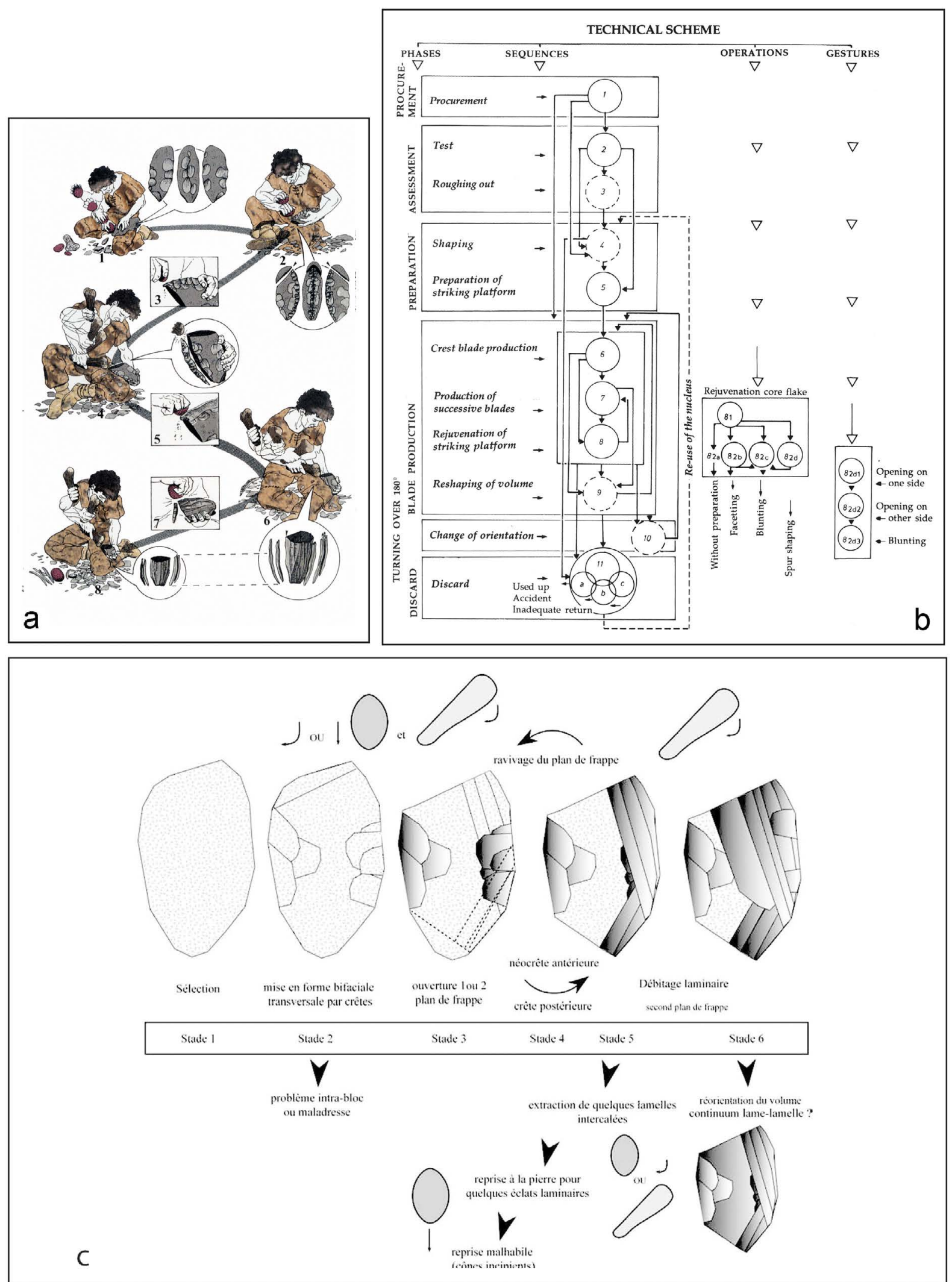

Figure 3. Différentes représentations du schème du débitage laminaire magdalénien : a) d’après G. Tosello (collectif 1991b: 29) ; b) schématisé par C. Karlin et S. Ploux (Karlin \& Julien 1994: 155, fig. 15.1) ; c) reformulé en DAO par M. Langlais (2013: 22, fig. 22).

Figure 3. Different representations of the laminar knapping scheme: a) drawn from G. Tosello (collective publication 1991b: 29); b) schematized by C. Karlin and S. Ploux (Karlin \& Julien 1994: 155, fig. 15.1); c) reformulated by M. Langlais in CAD (2013: 22, fig. 22). 
Ce ne sont plus alors les similitudes observées qui vont attirer notre attention, mais les variations entre les différentes chaînes opératoires reconstituées auxquelles il va falloir donner du sens (Ploux et al. 1992; Ploux \& Karlin 1994). Une réflexion sur le savoir-faire est conduite, c'est à dire sur l'ensemble des connaissances, expériences et techniques accumulées par une société (ce qui renvoie au schème) ou un individu (ce qui renvoie à ses compétences) (Figure 4). Des chercheurs américains se sont les premiers attachés à l'étude de la variabilité individuelle : dès 1974 ils cherchent lors d'un colloque, à découvrir la nature de la variabilité individuelle, à l'isoler et à voir dans quelle mesure elle peut être profitable à la recherche archéologique (Hill \& Gunn 1977; Hill 1978). En Europe, la problématique est reprise de manière très différente dans les années 80 , en particulier en France. Ce sont les travaux de Pigeot sur les séquences de débitage de l'unité U5 d’Etiolles (Pigeot 1983) : la reconnaissance d'habiletés variables et l'identification de la nature des maladresses la conduisent à proposer une grille dans l'acquisition progressive des principes de base en vigueur dans un débitage laminaire. De son coté, Ploux, d'abord sur des tailleurs expérimentaux (Ploux [Maîtrise 1979] 1980), puis sur le matériel de l'unité 36-M89 du niveau IV20 de Pincevent (Ploux 1989; Ploux \& Karlin 2014) cherche, à partir d'une analyse des performances, à différencier des tailleurs de compétence ou non comparable (Figure 5).

Plus nombreuses sont les unités identifiées au sein d'une chaîne opératoire, plus nombreuses sont les combinaisons de variables possibles, chacune pouvant être soumise à variations. La répétition de certaines variations dans un assemblage donné en fait des éléments du schème : elles lui donnent sa flexibilité qui doit répondre à la multiplicité des formes et des qualités des blocs travaillés, ce que Leroi-Gourhan attribuait au milieu extérieur « formé par les ressources minérales offertes à l'activité humaine ainsi que les propriétés des roches taillables dont (a déjà été) souligné la spécificité et la stabilité » (Pelegrin 2004: 152). Mais d'autres variations relèvent de causes extérieures à l'acte technique lui-même et conduisent à aborder le milieu intérieur, "c'est à dire les facteurs proprement humains à l'œuvre dans la taille » (Pelegrin 2004: 152) et le rôle du contexte social dans lequel s'ancre l'acte technique.

Toute séquence reconstituée est une performance réalisée par un individu dans un contexte précis. Il devient possible de rendre vie à cet individu en analysant et en attribuant une valeur à chaque moment de la chaîne opératoire dont il est responsable. En effet cette performance présente des variations, mêlées à l'application des grands principes, qui résultent des compétences mises en œuvre, des capacités du tailleur à construire un projet, à évaluer le potentiel de son bloc, à suivre le schéma imaginé, à manier ses percuteurs, ou à faire des choix efficaces à des moments clés (Tableaux $1 \& 2$, Figure 6). Ploux élabore une grille de lecture des performances de différents tailleurs expérimentaux puis l'applique à du matériel archéologique en mesurant la distance qu'il y a entre un tailleur contemporain qui a appris adulte et réalise sa performance dans un objectif scientifique et un tailleur préhistorique qui taille depuis sa plus tendre enfance pour subvenir à ses besoins et à ceux de son groupe (Ploux 1991). Tout tailleur «met en œuvre un savoir-faire opératoire et un savoir-faire moteur (Pelegrin 1991a, 1991b, 1993). Le premier correspond aux opérations cognitives effectuées au cours de la taille. Le second fait référence à l'activité motrice en jeu dans la taille de la pierre. » (Bril \& Roux 1993). À ces deux niveaux, les variations observées entre la chaîne opératoire modèle et la performance réalisée sont un chemin d'accès aux compétences des tailleurs. Ce travail sur la reconnaissance des individus à partir de la lecture des chaînes opératoires a été conduit à Etiolles (Pigeot [thèse 1983] 1987) et approfondi à Pincevent dans une collaboration entre l'équipe Tixier, à laquelle appartenait Ploux, et celle de LeroiGourhan (Ploux 1989; Bodu et al. 1990). 


\section{Assemblage $A$}

Réactions spécifiques

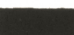

Réactions partiellement partagées

एण एण पय

Réactions communes
Individu $Y$,

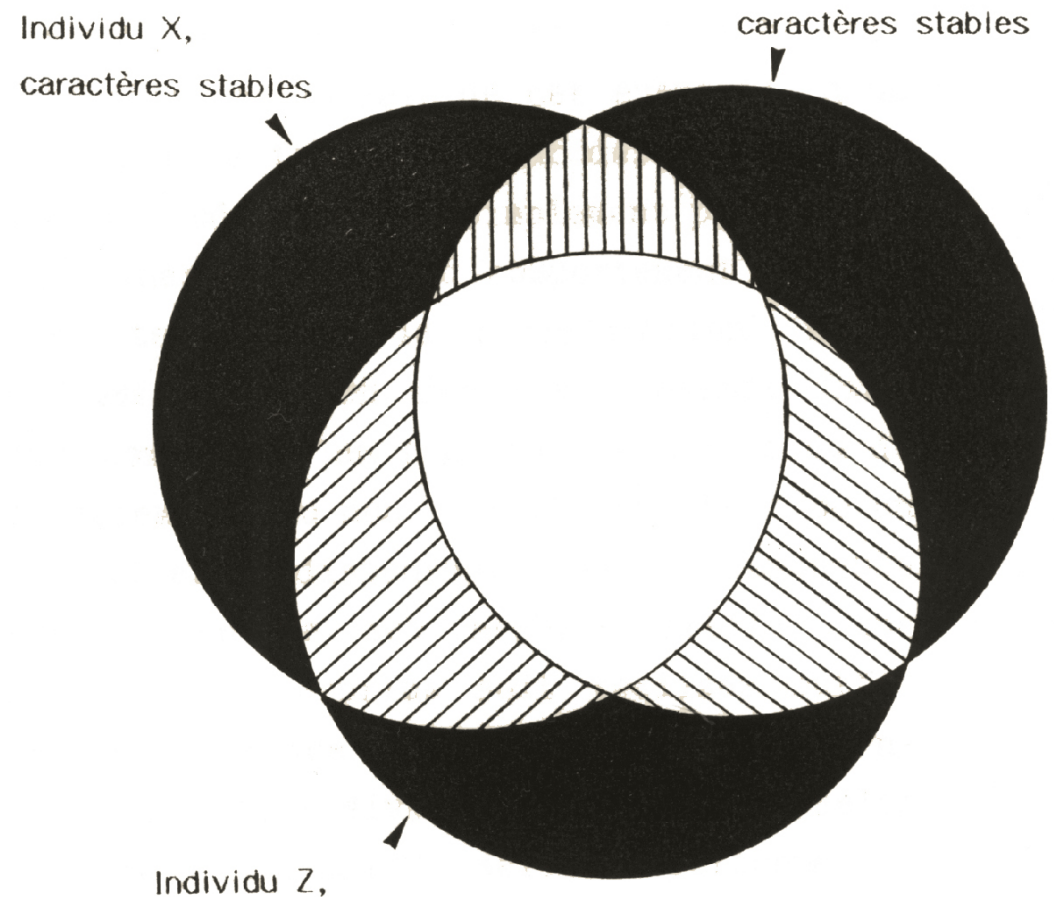

caractères stables

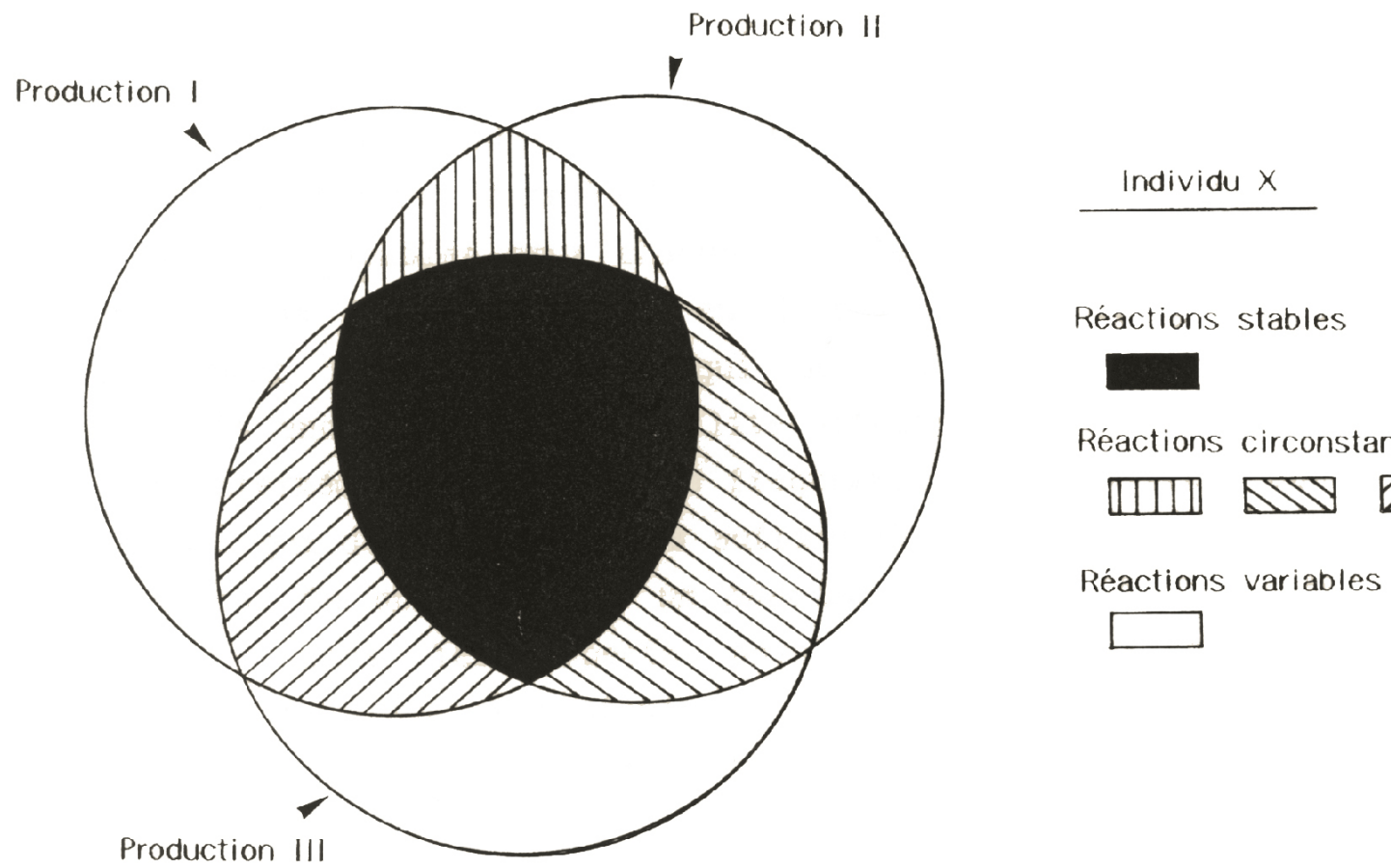

Figure 4. Schématisation élaborée par S. Ploux a) des différents degrés de l'uniformisation intra-communautaire marquant un assemblage et b) des marges de stabilité et de variabilité dans la production d'un même individu (Ploux 1989: 26, fig. 7).

Figure 4. Scheme created by S. Ploux to express a) the various degrees of intra-group uniformization of lithic assemblages; and b) the margins between stability and variability within the production of an individual (Ploux 1989: 26, fig. 7). 


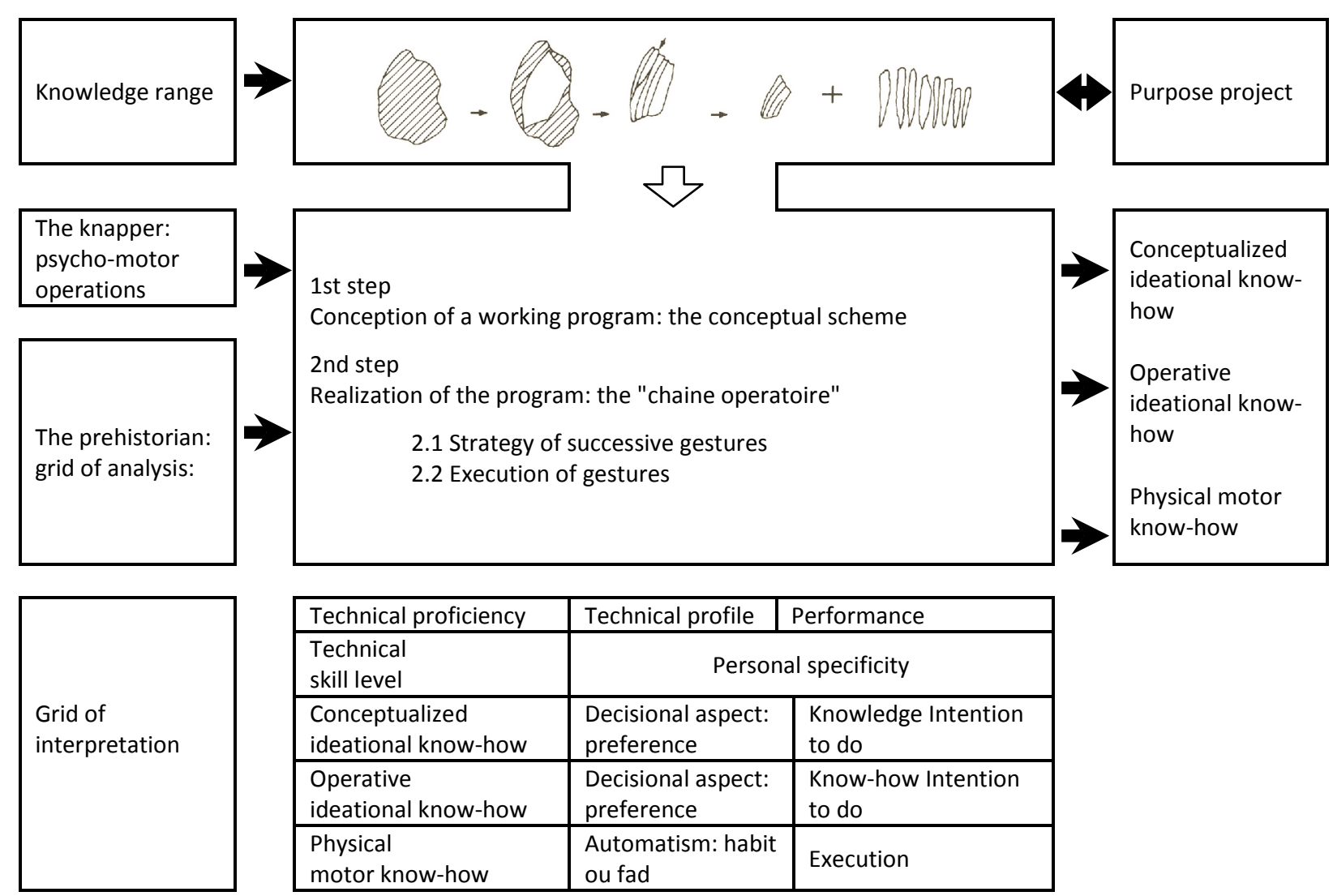

Figure 5. Représentation élaborée par S. Ploux des différents niveaux d’analyse des séquences de débitage magdalénien : connaissance, savoir-faire, intention de faire, exécution ainsi que la grille d’interprétation du préhistorien (Karlin \& Julien 1994: 160, fig. 15.3).

Figure 5. Analytical grid built by S. Ploux of the various levels of analysis of Magdalenian knapping sequences: knowledge, know-how, production intention, carrying out and interpretation grid used by the prehistorian (Karlin \& Julien 1994: 160, fig. 15.3).

À Pincevent, Ploux distingue deux groupes de tailleurs qui vont conduire leur réalisation sur des objectifs différents, élément qui influe bien évidemment sur la conduite de la réduction du rognon. Le premier travaillera en vue d'une production efficace d'outils ou d'armes. Le second taillera en vue d'intégrer l'apprentissage d'une technique qu'il doit maîtriser pour vivre. La distinction de ces deux groupes, productifs et apprentis, conduit à regrouper dans chacun des chaînes opératoires comparables pour lesquelles il reste ensuite à mesurer les niveaux des connaissances acquises et mises en œuvre. À Verberie, M. Langlais, après F. Janny, met en avant, plus que la participation à la productivité, une hiérarchie progressive dans l'acquisition des compétences (Langlais 2013: 35) (Tableau 2, Figure 6).

Le groupe des tailleurs productifs permet d'aborder la gestion de cette activité (Audouze et al. 1988). Certains maîtrisent parfaitement la technique, savent changer de percuteur en fonction du produit voulu ou changent simplement la manière de l'utiliser et réalisent exactement ce qu'ils avaient envisagé (Figure 7). Entre leurs mains arrivent les meilleurs blocs. Ils fabriquent les lamelles standardisées qui armeront les sagaies et des lames qui seront le support d'outils domestiques ou circuleront dans le campement. D'autres auront plus ou moins de mal à mettre en œuvre leur projet initial et devront en cours de route réorienter leur travail en fonction des aléas de la matière première et de leur incapacité à les maîtriser (Figure 8). Eux se contenteront d'une fabrication de supports d'outils domestiques plus ou moins réguliers. Dans une unité d'habitation à la fois bien circonscrite et cohérente il est même possible d'affiner et de repérer des individus à partir de variations dans les performances au sein d'un même niveau de compétence. Ce à quoi peut s'ajouter l'observation de quelques tics 
idiosyncratiques qui caractérisent certaines chaînes opératoires et identifient clairement leur auteur (Ploux et al. 2014).

Tableau 1. Critères permettant de reconnaître une hiérarchie des niveaux de savoir-faire dans l'organisation des débitages laminaires et d’identifier des auteurs à compétence différente : pour l'unité U5 d'Étiolles N. Pigeot classifie les performances à partir de connaissances maîtrisées identifiées sur les remontages, les tailleurs les plus performants étant en haut du tableau (D’après Pigeot 1990: 133, fig. 4).

Table 1. Criteria for identifying a hierarchy of know-how levels in the organization of laminar productions, and for identifying knappers of various levels of competence: for unit U5 at Étiolles N. Pigeot classifies the performances using identified and mastered knowledges reading the refitings, the best knappers at the top of the table (Drawn from Pigeot 1990: 133, fig. 4).

\begin{tabular}{|c|c|c|c|c|c|c|c|c|}
\hline $\begin{array}{l}\text { Lecture de l'acquisition graduelle } \\
\text { des principes techno- } \\
\text { économiques dans le débitage } \\
\text { laminaire sur des nucléus de U5 }\end{array}$ & $\begin{array}{l}\text { Nucléus } \\
\text { autres }\end{array}$ & N333 & N216 & $\begin{array}{c}296 \\
\text { N274 }\end{array}$ & N14 & N236 & $\begin{array}{l}\text { N257 } \\
\text { N481 }\end{array}$ & $\begin{array}{l}\text { N263 } \\
\text { N400 } \\
\text { N518 }\end{array}$ \\
\hline $\begin{array}{l}\text { Travaillés avec un angle de frappe } \\
\text { égal ou inférieur à } 900\end{array}$ & & & & & & & & \\
\hline $\begin{array}{l}\text { Conception du couple plan de } \\
\text { frappe ou table laminaire }\end{array}$ & & & & & & & & \\
\hline $\begin{array}{l}\text { Préparation du bord du plan de } \\
\text { frappe (éperon ou facettage) }\end{array}$ & \multicolumn{4}{|c|}{ éperon } & & ceté & & \\
\hline $\begin{array}{l}\text { Entretien du volume du nucléus ou } \\
\text { réorganisation }\end{array}$ & \multicolumn{4}{|c|}{ complexe } & élém & entaire & & \\
\hline Extraction des lames & \multicolumn{4}{|c|}{ Longues et régulières } & & urtes & & \\
\hline \multicolumn{9}{|l|}{ productivité économique } \\
\hline \multicolumn{9}{|l|}{$\begin{array}{l}\text { Pas d'insistance inutile en cours } \\
\text { d'opération }\end{array}$} \\
\hline \multicolumn{9}{|l|}{$\begin{array}{l}\text { Pas d'insistance inutile à la fin de } \\
\text { l'opération }\end{array}$} \\
\hline $\begin{array}{l}\text { Un débitage élaboré (maîtrise des } \\
\text { principes et savoir-faire) }\end{array}$ & & & & & & & & \\
\hline
\end{tabular}

Le second groupe permet d'aborder l'acquisition d'un savoir-faire et la gestion de la transmission (Figure 9). "L'apprentissage de la taille dans un milieu plus ou moins clos augmente les chances d'identité des divers caractères, allant de la position globale du corps au type de préparation préférentielle du plan de frappe » (Ploux [maîtrise 1979] 1980: 159). Plus les compétences seront faibles, plus la marge de variations sera importante à tous les niveaux, construction du projet ou même son absence, efficacité motrice, capacité à rattraper un problème. Si dans le groupe des tailleurs productifs la durée relativement courte de l'occupation ne laisse pas supposer une évolution visible des compétences, dans celui des apprentis la relative instabilité des savoir-faire autorise une progression sensible.

Lors de l'apprentissage, l'observation permet d'intégrer par imprégnation ce qui est visible, d'autant plus facilement que la taille du silex se fait au vu et au su de tous. Mais les notions abstraites comme la manière de conduire la gestion de l'exploitation en prévoyant l'évolution du volume peuvent faire l'objet d'une transmission dirigée. Il en va de même quant à la manière de se poser pour travailler afin que le geste puisse se développer efficacement. Une leçon a été observée à Pincevent (Ploux \& Karlin 2014; Ploux et al. 2014: 246-248) (Figure 10). Cette mise en évidence de l'acquisition d'un savoir a conduit à proposer la présence de jeunes apprentis à Etiolles comme à Pincevent (Pigeot 1986; [Thèse 1983] 1987; 1990; Karlin \& Pigeot 1989; Ploux \& Karlin 2014; Ploux et al. 2014: 246-248) ainsi qu'à Verberie (Audouze \& Janny 2009: 171-172; Langlais 2013: 34-44) ou encore à 
Monruz-Neuchâtel (Cattin 2012: 250-253). Et commence a être évoquée la possibilité, à travers la technnologie, d'une approche cognitive de quelques aspects de la pensée des tailleurs préhistoriques (Pelegrin 1985). Cette approche cognitive va devenir un des thèmes majeurs de l'équipe Préhistoire et Technologie.

Tableau 2. Critères permettant de reconnaître une hiérarchie des niveaux de savoir-faire dans l'organisation des débitages laminaires et d’identifier des auteurs à compétence différente : à Verberie F. Janny classifie à partir de quelques critères et commence en haut par les moins performants (Audouze \& Janny 2009: 173, pl. XXIV). Toujours à Verberie, M. Langlais classe lui de gauche à droite, du moins au plus performant (Langlais 2013: 36, fig. 35).

Table 2. Criteria for identifying a hierarchy of know-how levels in the organization of laminar productions, and for identifying knappers of various levels of competence: at Verberie F. Janny classifies from some criteria and the less efficient are at the top of the grid (Audouze \& Janny 2009: 173, pl. XXIV); at Verberie again, M. Langlais sorts the knappers from left to right, from the less efficient to the most efficient knappers (Langlais 2013: 36, fig. 35).

\begin{tabular}{|c|c|c|c|c|c|}
\hline $\begin{array}{l}\text { niveaux de } \\
\text { compétence } \\
\text { des tailleurs du } \\
\text { Il1 de Verberie }\end{array}$ & $\begin{array}{l}\text { type de } \\
\text { percussion }\end{array}$ & $\begin{array}{l}\text { négatifs visibles sur le } \\
\text { nucleus }\end{array}$ & résultats & $\begin{array}{l}\text { nombre de } \\
\text { nucleus } \\
\text { (total 164) }\end{array}$ & auteurs \\
\hline $\begin{array}{l}\text { débitage } \\
\text { autonome }\end{array}$ & $\begin{array}{l}\text { percuteur dur } \\
\text { en pierre }\end{array}$ & $\begin{array}{l}\text { endommagement du } \\
\text { plan de frappe }\end{array}$ & $\begin{array}{l}\text { production } \\
\text { nulle }\end{array}$ & 10 & $\begin{array}{l}\text { très jeune } \\
\text { sans } \\
\text { contrôle } \\
\text { psycho- } \\
\text { moteur }\end{array}$ \\
\hline $\begin{array}{l}\text { débitage } \\
\text { secondaire sur } \\
\text { un nucleus } \\
\text { déjà débité }\end{array}$ & $\begin{array}{l}\text { percuteur dur } \\
\text { en pierre }\end{array}$ & massacre & $\begin{array}{l}\text { aucun } \\
\text { enlèvement } \\
\text { correct (éclat } \\
\text { ou lame) }\end{array}$ & 10 & très jeune \\
\hline $\begin{array}{l}\text { débitage } \\
\text { autonome }\end{array}$ & $\begin{array}{l}\text { percuteur dur } \\
\text { en pierre }\end{array}$ & $\begin{array}{l}\text { endommagement du } \\
\text { plan de frappe }\end{array}$ & $\begin{array}{l}\text { quelques éclats } \\
\text { réfléchis qui } \\
\text { ruinent le } \\
\text { nucleus }\end{array}$ & 4 & débutant \\
\hline $\begin{array}{l}\text { débitage } \\
\text { autonome }\end{array}$ & $\begin{array}{l}\text { percuteur } \\
\text { organique }\end{array}$ & $\begin{array}{l}\text { préparation du plan de } \\
\text { frappe et de la crête } \\
\text { correcte }\end{array}$ & $\begin{array}{l}\text { pas de lame } \\
\text { ou au mieux } \\
\text { une lame } \\
\text { réfléchie }\end{array}$ & 14 & apprenti \\
\hline $\begin{array}{l}\text { débitage } \\
\text { secondaire }\end{array}$ & $\begin{array}{l}\text { percuteur } \\
\text { organique }\end{array}$ & $\begin{array}{l}\text { manque de soin ds la } \\
\text { préparation (savoir } \\
\text { acquis mais pas encore } \\
\text { le savoir-faire) }\end{array}$ & $\begin{array}{l}\text { une lame } \\
\text { réfléchie }\end{array}$ & 1 & $\begin{array}{l}\text { aprenti } \\
\text { manquant } \\
\text { encore } \\
\text { d'expérience }\end{array}$ \\
\hline $\begin{array}{l}\text { débitage } \\
\text { autonome }\end{array}$ & $\begin{array}{l}\text { percuteur de } \\
\text { pierre tendre } \\
\text { ou percuteur } \\
\text { organique }\end{array}$ & préparation soignée & $\begin{array}{l}\text { séries de } \\
\text { lames }\end{array}$ & 121 & $\begin{array}{l}\text { tailleur } \\
\text { compétent }\end{array}$ \\
\hline $\begin{array}{l}\text { débitage } \\
\text { autonome }\end{array}$ & $\begin{array}{l}\text { percuteur de } \\
\text { pierre tendre } \\
\text { ou percuteur } \\
\text { organique }\end{array}$ & $\begin{array}{l}\text { préparation très } \\
\text { soignée du bloc et } \\
\text { anticipation des } \\
\text { difficultés techniques }\end{array}$ & $\begin{array}{l}\text { grand nombre } \\
\text { de longues } \\
\text { lames }\end{array}$ & 2 & $\begin{array}{l}\text { le meilleur } \\
\text { tailleur }\end{array}$ \\
\hline
\end{tabular}

Quelque soit le niveau du tailleur, chaque performance se développe dans l'espace et le temps. À Etiolles, l'espace des tentes est hiérarchiquement structuré en fonction des compétences: "Les meilleurs tailleurs s'installent autour du foyer. Derrière dans la zone médiane travaillent les tailleurs occasionnels, tandis qu'à la périphérie (...) les jeunes s'entraînent à parfaire leur maîtrise technique » (Karlin et al. 1992: 1106-1115) (Figure 11a). À Verberie les meilleurs tailleurs occupent les postes de travail autour du foyer D1, le dos au 
vent ; les autres taillent un peu plus loin (Figure 11b). À Pincevent cette hiérarchie n'existe pas et chacun peut tailler n'importe où, que ce soit dans les résidences (Figure 11c) ou les ateliers (Figure 11d) (Karlin 1992: 145, Julien \& Karlin 2014).

PROCESSUS D'ACQUISITION DE LA TECHNIQUE DE TAILLE A VERBERIE

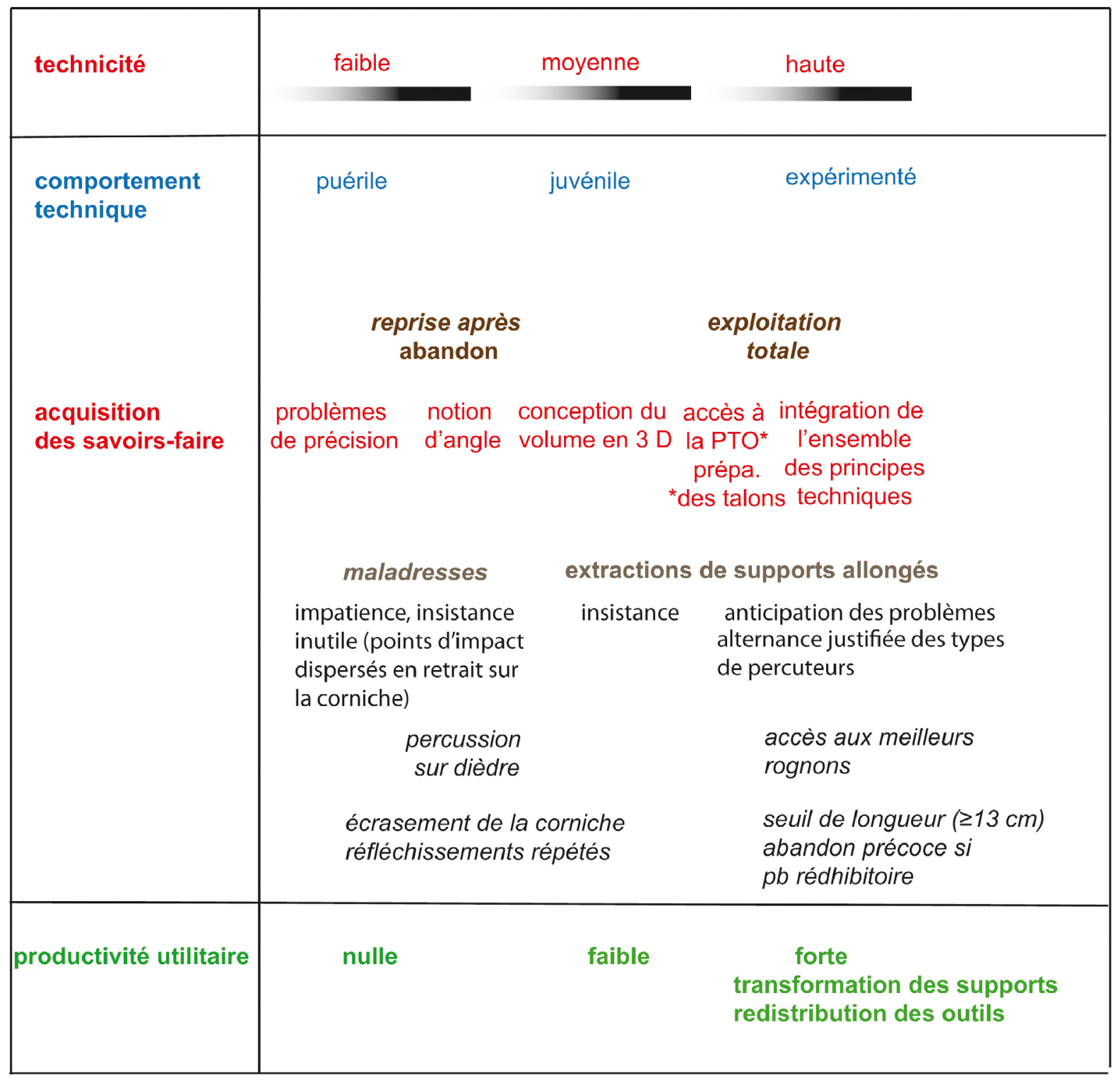

* PTO $=$ percussion tangentielle organique

Figure 6. Critères permettant de reconnaître une hiérarchie des niveaux de savoir-faire dans l'organisation des débitages laminaires et d'identifier des auteurs à compétence différente : à Verberie F. Janny classifie à partir de quelques critères et commence en haut par les moins performants (Audouze \& Janny 2009: 173, pl. XXIV). Toujours à Verberie, M. Langlais classe lui de gauche à droite, du moins au plus performant (Langlais 2013: 36, fig. 35).

Figure 6. Criteria for identifying a hierarchy of know-how levels in the organization of laminar productions, and for identifying knappers of various levels of competence: at Verberie F. Janny classifies from some criteria and the less efficient are at the top of the grid (Audouze \& Janny 2009: 173, pl. XXIV); at Verberie again, M. Langlais sorts the knappers from left to right, from the less efficient to the most efficient knappers (Langlais 2013: 36, fig. 35).

Par ailleurs, des changements de lieux au cours de la même séquence opératoire correspondent de fait à des ruptures temporelles plus ou moins longues et marquent le plus 
souvent un changement de phase dans la réalisation du travail. Dans le cas de la taille du silex, et lorsqu'il ne s'agit pas d'un atelier sur le gite, la première rupture est entre l'approvisionnement et l'exploitation du bloc sur le lieu de vie. Mais une première phase du travail peut avoir lieu sur le gite même, simple test ou première ébauche pour ne rapporter que de la matière première rentable. Lorsque l'opération se passe ou se poursuit dans le campement le tailleur peut changer de place (Karlin 1992; Bodu 1993), comme par exemple se mettre à quelque distance pour dégrossir son bloc afin de ne pas encombrer l'espace collectif (habitation ou atelier) de déchets polluants puis y revenir pour réaliser le plein débitage (Bodu et al. 2014: 412-414) (Figure 12). À l'inverse, on conçoit difficilement que certaines opérations puissent être exécutées en discontinuité, par exemple la mise en place d’un éperon et l'extraction de la lame qu'il prépare.

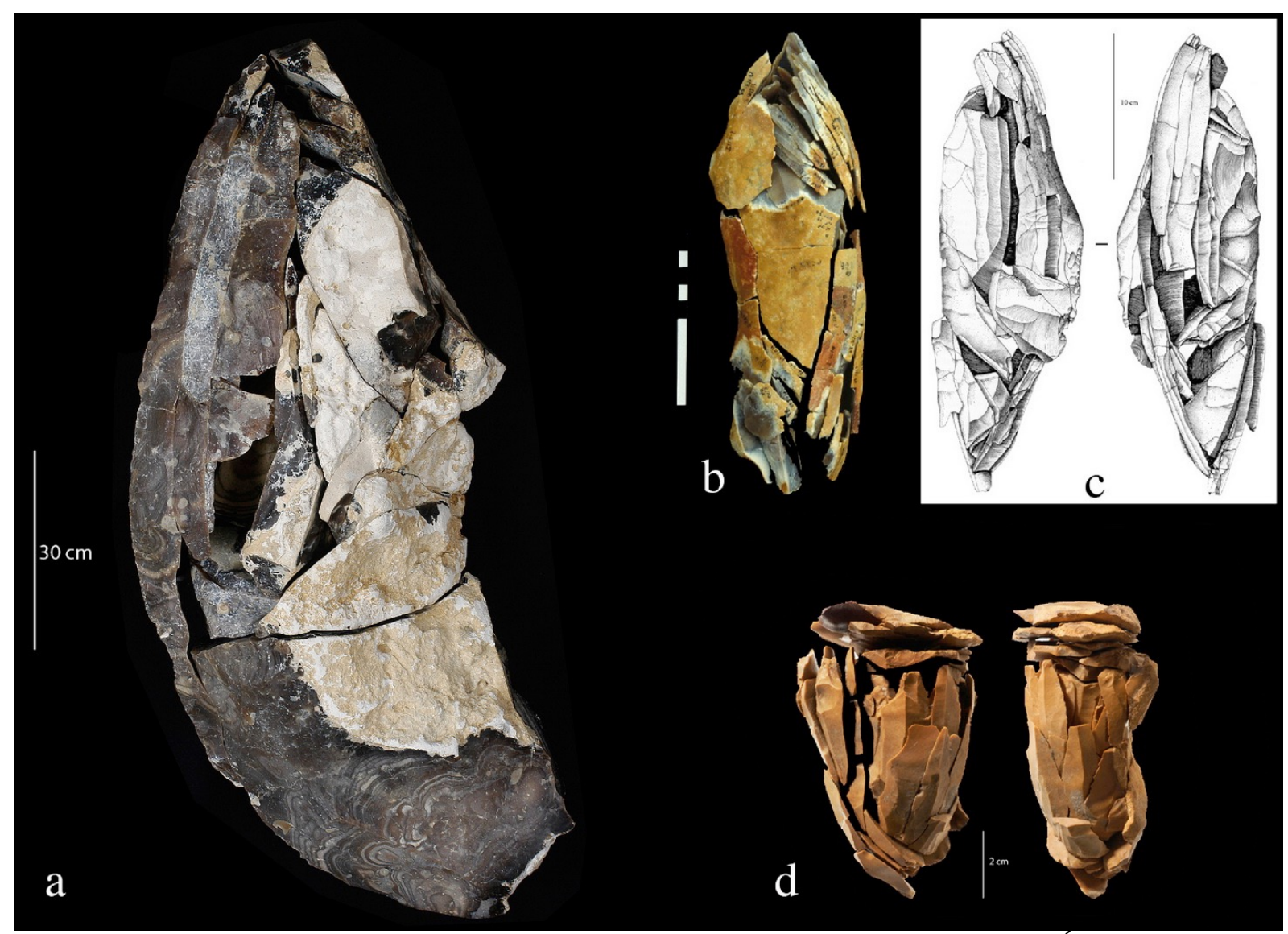

Figure 7. Quatre exemples de productions de tailleurs magdaléniens très expérimentés : a) Étiolles offre parmi les plus grands débitages de longues lames du Magdalénien sur de très gros bloc (Olive et al. 1991: 7, fig.3, cliché P. Masson) ; b) Pincevent niveau IV20, séquence 36-G119.4 (Bodu et al. 2014: 401, fig. 28, cliché P. Bodu) ; c) Verberie, séquence RL 289 (Audouze \& Cattin 2011: 116, fig. 8, dessin Y. Baele); c) Montruz, séquence MP109, nucléus P46.1 (Cattin 2012: 73, fig. 60, cliché Marc Juillard, Laténium). Sur les exemples b et c on observe qu'un travail en opposé sur des blocs de bonne dimension permet d'entretenir naturellement la courbure longitudinale du bloc ou carène. L'exemple d montre, sur un bloc de plus petite dimension, un entretien de la courbure transversale, ou cintre, simplement par la succession des enlèvements de lames.

Figure 7. Four examples of productions by highly experienced knappers: a) Some of the longest blades debitages on very large blocks have been found at Étiolles (Olive et al. 1991: 7, fig.3, photo P. Masson); b) Pincevent level IV20, refit 36-G119.4 (Bodu et al. 2014: 401, fig. 28, photo P. Bodu); c) Verberie, sequence RL289 (Audouze \& Cattin 201: 116, fig. 8, drawing Y. Baele); d) Montruz, refit MP109, core P46.1 (Cattin 2012: 73, fig. 60, photo Marc Juillard, Laténium). Refits a and b show how knapping from both ends allows to keep the proper longitudinal curving of the removal surface. In the smaller refitted block d, removing the successive blades is enough to keep the proper bending (or transversal curvin) of the removal surface. 

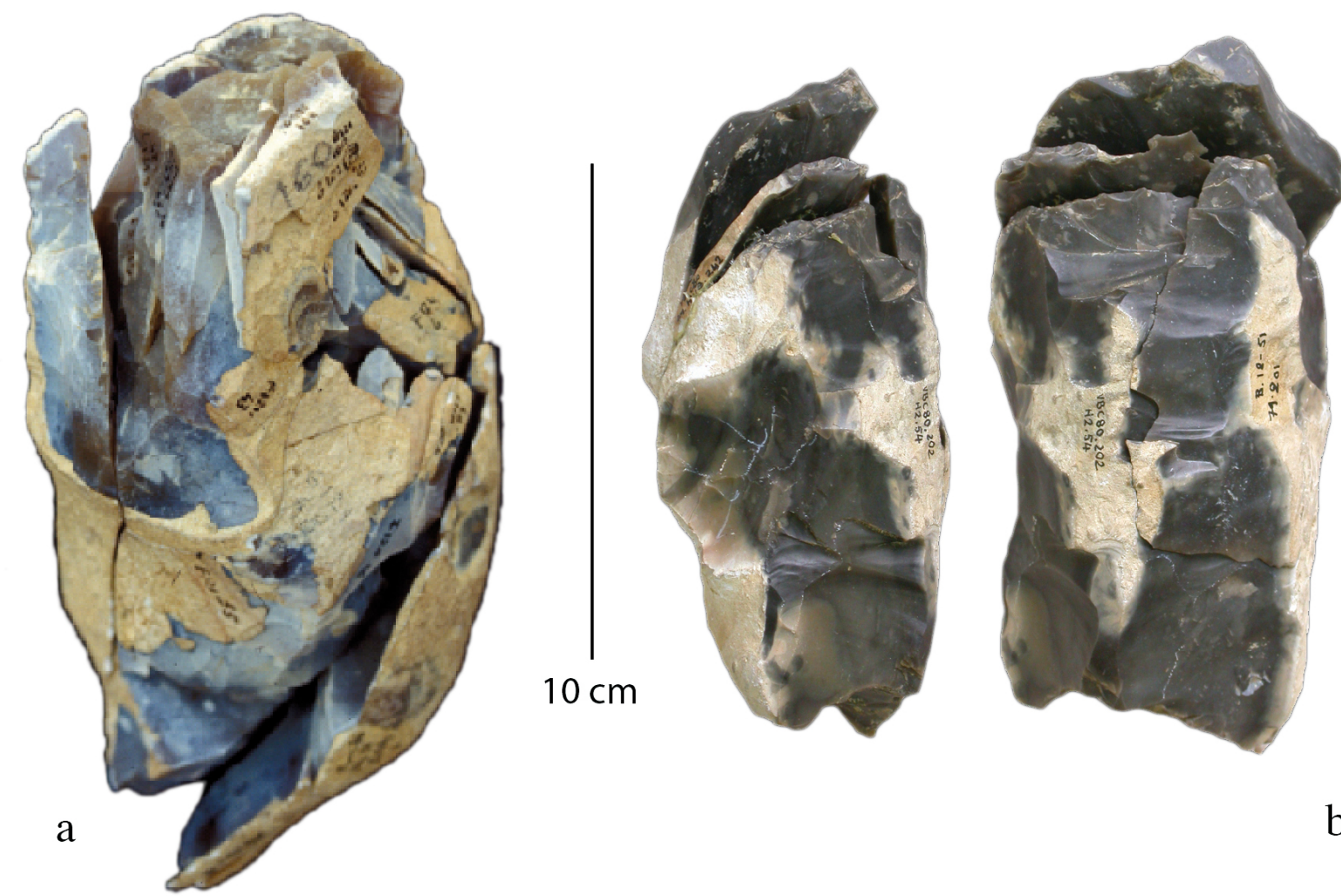

$\mathrm{b}$

Figure 8. Deux exemples de productions de tailleurs magdaléniens simplement compétents : a) Pincevent, niveau IV20, séquence 36-G120.8c (cliché P. Bodu) ; b) Verberie, séquence laminaire RL 253 (Langlais 2013: 38, fig. 38 ; cliché de M. Langlais).

Figure 8. Two examples of productions by just competent knappers: a) Pincevent, level IV0, sequence 36G120.8 (photo P. Bodu); b) Verberie, laminar sequence RL253 (Langlais 2013: 38, fig. 38; photo by M. Langlais).

Enfin, certaines séquences opératoires ont montré des ruptures si importantes de niveau de compétence entre la construction du projet et sa mise en œuvre puis la phase finale d'exploitation et l'état du nucléus à son abandon que ces deux phases ne pouvaient être le fait d'un même individu. La répétition de ces ruptures nous a conduit à constater que des blocs abandonnés après une première exploitation par des tailleurs productifs étaient repris à fin d'entraînement par des apprentis. S'il s'agit bien d'un seul et même bloc, il nous semble nécessaire de distinguer deux chaînes opératoires différentes car conduites par des individus différents, sur des objectifs différents, dans des temps et des lieux souvent différents. (Ploux \& Karlin 2014: 126) (Figure 13).

La description d'une chaîne opératoire doit préserver la cohérence interne de ses différentes étapes et aussi permettre d'exprimer ses rapports avec d'autres chaînes opératoires. Car plusieurs peuvent s'articuler en système, ce que Lemonnier désignait par processus technique. Pour fabriquer une arme de chasse, il faudra préparer des lamelles à dos par débitage puis façonnage. De manière indépendante il faudra préparer des pointes de sagaies à partir de fragments de baguettes extraites dans des bois de renne. De manière tout aussi indépendante il faudra préparer des hampes en bois. Et ces trois chaînes, qui peuvent ou non être réalisées par des auteurs différents, vont à un moment donné converger pour une chaîne de fabrication d'arme de chasse qui va sceller ces trois éléments en utilisant de la colle et des liens (Julien et al. 2014: 502-503). 

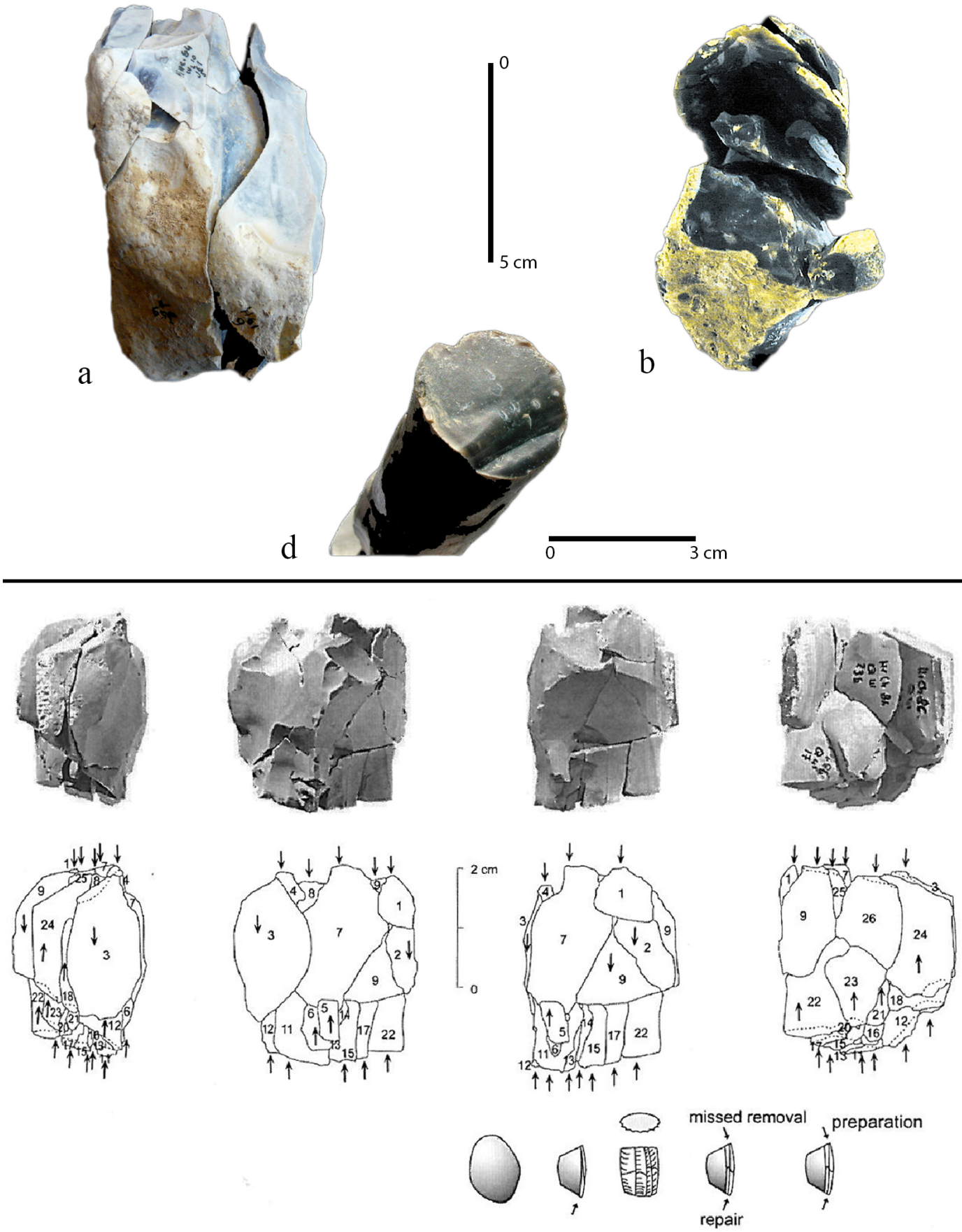

C

succession of missed removals and repairing preparations

Figure 9. Quatre exemples d'exercices de tailleurs apprentis identifiés par l'absence de préparation qui réduit la séquence à quelques produits ou ne donne que des éclats au lieu des lames espérées : a) Pincevent, niveau IV20, séquence 17-J59.1 (Julien et al. 2014: 531, fig. 17, cliché C. Karlin) ; b) Verberie séquence 190.1 (cliché M. Langlais) ; c) Champréveyres (Audouze \& Cattin 2011: 117, fig. 9, dessin de M.I. Cattin, cliché Y. André) ; d) Verberie, nucléus digité O1.9 portant jusqu'au centre de la surface des traces maladroites de percussions à la pierre dure qui ne pouvaient donner lieu à aucun enlèvement ; s’agit-il là d’un jeu d'enfant (cliché F. Audouze) ? Figure 9. Four examples of trials by apprentice knappers, identified by the lack of preparation resulting in a short sequence reduced to a few products or yielding a few flakes instead of the expected blades: a) Pincevent level IV20, sequence 17-J59.1 (Julien et al. 2014: 531, fig. 17, photo C. Karlin); b) Verberie séquence 190.1 (photo M. Langlais); c) Champréveyres (Audouze \& Cattin 2011: 117, fig. 9, drawing by M.I. Cattin, photo Y. André); d) Verberie, finger-like core O1.9 exhibiting in the middle of the striking platform unskilled traces of hard hammer percussion that could not result in any kind of flake extraction; could it be a child's play (photo by F. Audouze)? 


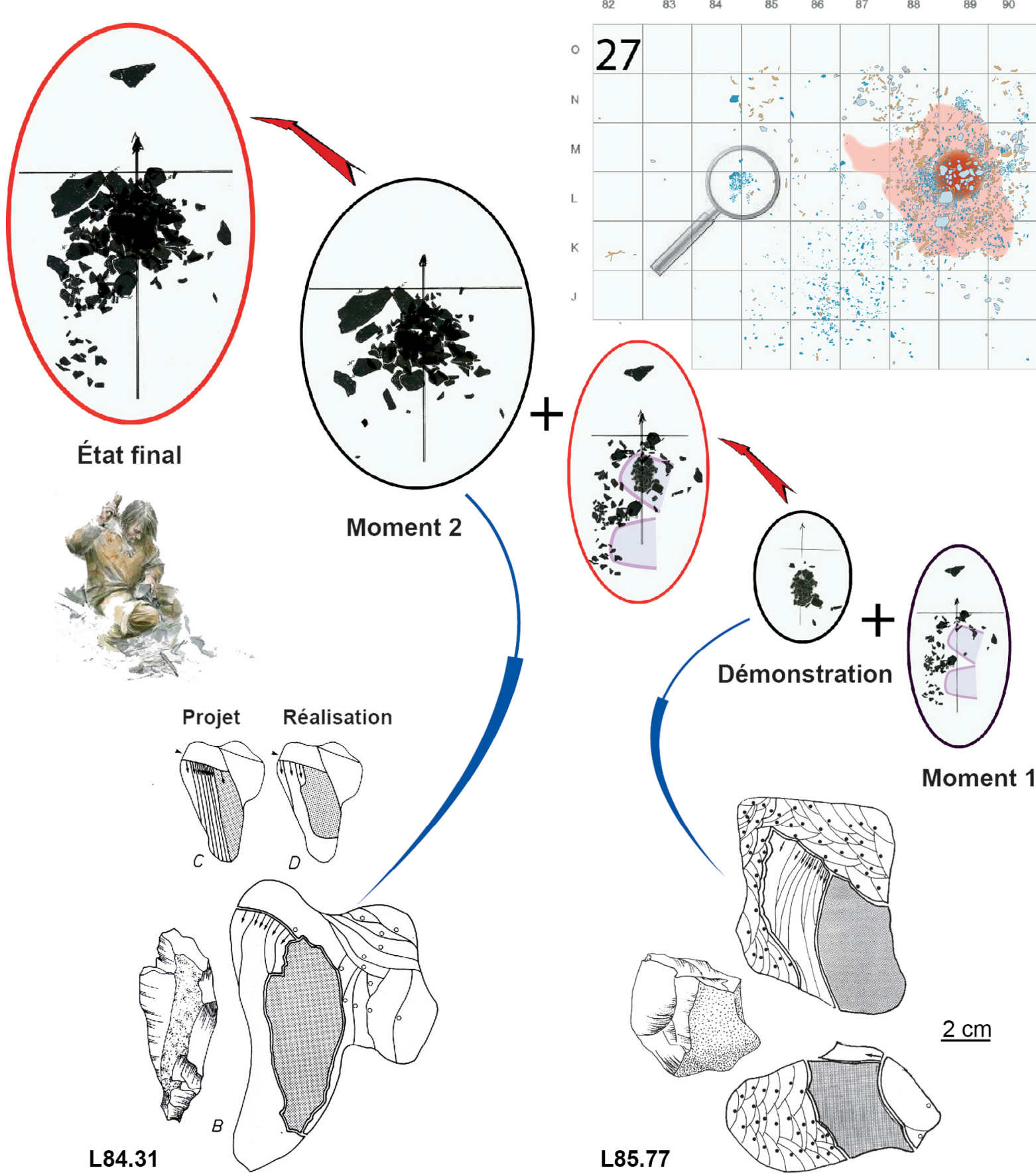

Figure 10. À Pincevent une leçon a été mise en évidence. L'apprenti a rassemblé quelques rognons peu adaptés à la taille et semble se mettre à genoux pour s'exercer. Dans le moment 1 on observe un large éparpillement des déchets autour des genoux. L'exercice est interrompu par un tailleur expérimenté qui fait une démonstration de la manière dont il faut penser l'évolution du volume. Les produits de sa démonstration sont pafaitement concentrés et tombent à l'emplacement d'un genou de l'apprenti qui a dû se reculer. Dans le moment 2 la production de l'apprenti est beaucoup plus concentrée car la position doit être meilleure. Et le saut qualitatif étant important, on peut penser que le tailleur expérimenté continue de prodiguer ses conseils à l'apprenti (Ploux et al. 2014: 247, fig. 29, DAO M. Hardy).

Figure 10. A knapping teaching was identified at Pincevent. An apprentice gathered several flint blocks poorly adapted to knapping, and probably kneeled to exercise. During moment 1 , he makes a wide scattering of flint refuse around his or her knees. An experienced knapper interrupts the exercise and he demonstrates how to conceive the evolution of the volume. The products from his demonstration are perfectly packed and fall where one of the apprentice's knee was, as he had probably to step back. In moment 2, the apprentice's products are much more packed because his position is probably better. One can think that the experienced knapper kept on advising the apprentice because the qualitative progress is big (Ploux et al. 2014: 247, fig. 29, CAD M. Hardy). 


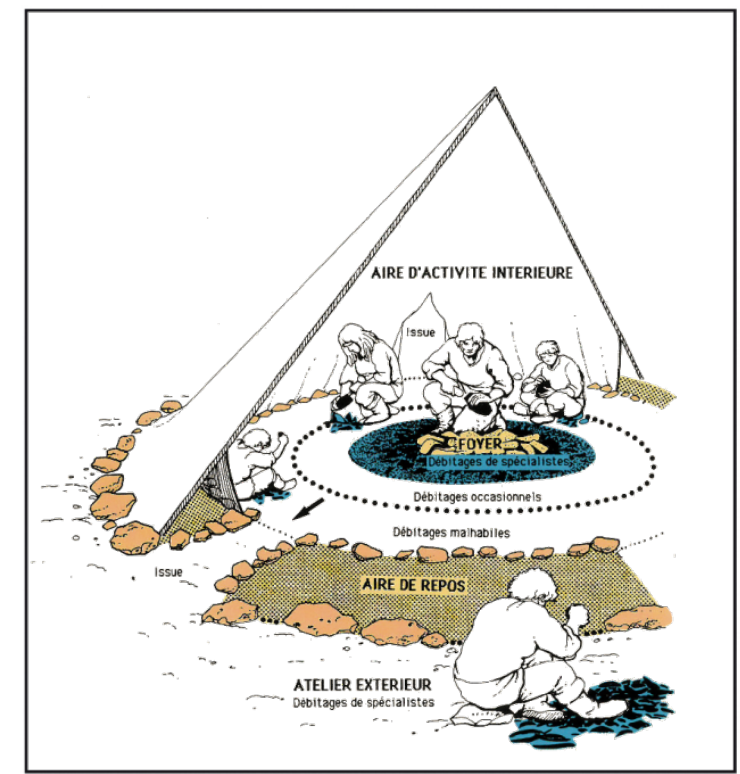

a

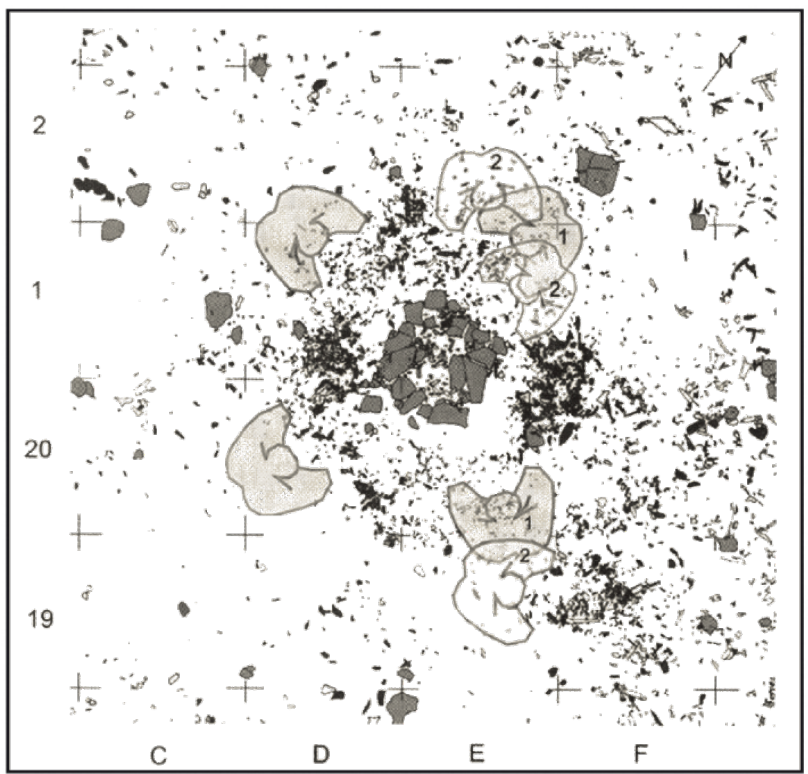

$\mathrm{b}$
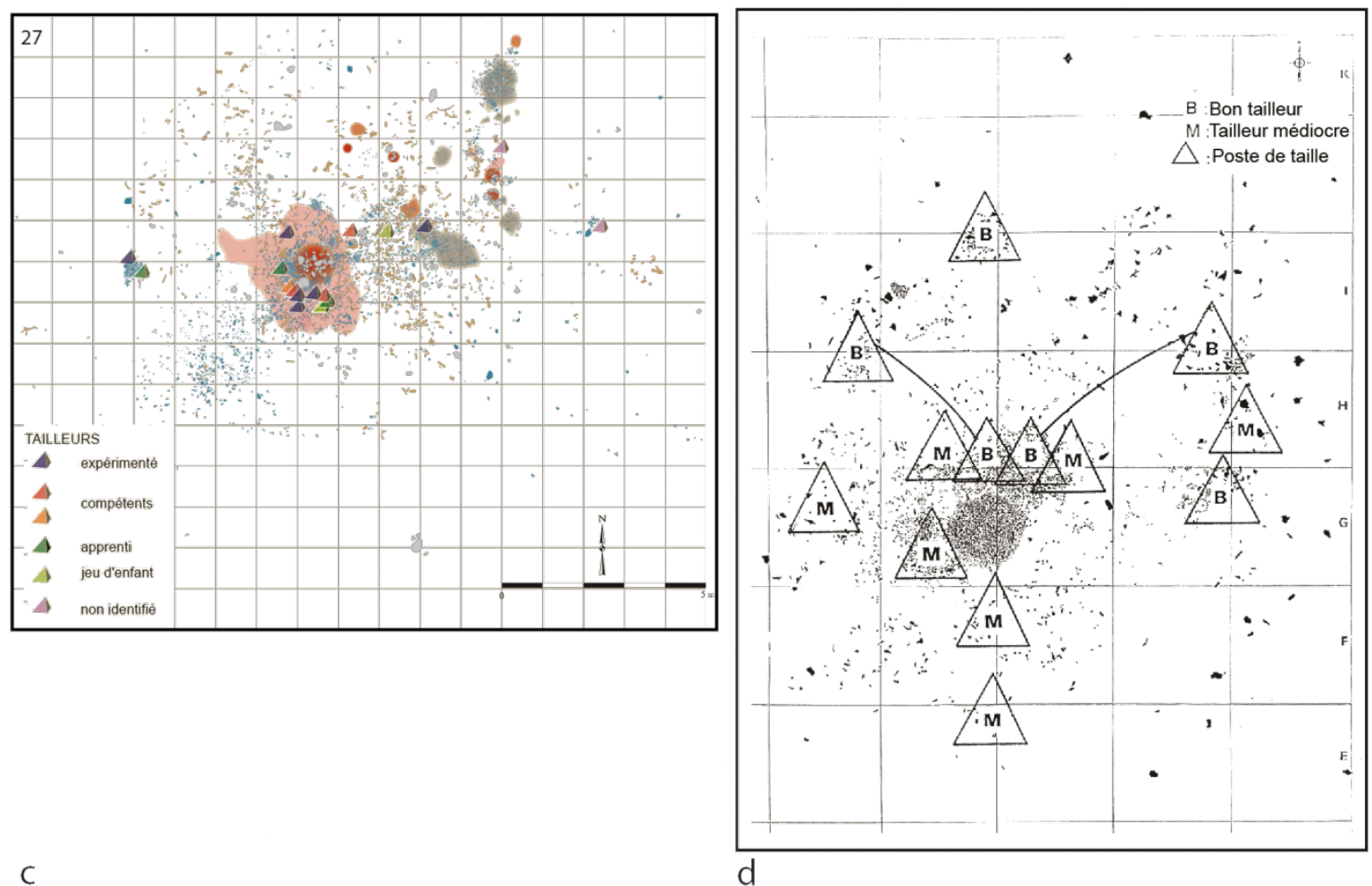

C

Figure 11. a) à Etiolles l'espace est structuré en fonction des compétences (Coll. 1991: 60, fig. 69, dessin de G. Tosello) ; b) à Verberie, les meilleurs tailleurs travaillent autour du foyer et peuvent changer de position au cours du temps sans pour autant s'éloigner (Audouze 2010: 151, fig. 3) ; c) À Pincevent chacun peut tailler où il veut, que ce soit dans les habitations, ici l'unité 27-M89 du niveau IV20 (Ploux et al 2014: 251, fig. 31, plan de M. Hardy) ou d) dans les unités techniques comme ici 36-G121 (Bodu et al 2014: 413, fig. 35, dessin de D. Molez). Figure 11. a) The organization of space is structured according to competence (Coll. 1991: 60, fig. 69, drawing by G. Tosello); b) at Verberie, the best knappers work around the hearth and can change position through time without moving away from the hearth (Audouze 2010: 151, fig. 3); c) at Pincevent, everybody can knap wherever he or she wants, whether in a habitation unit, here unit 27-M89 in level IV20 (Ploux et al 2014: 251, fig. 31, map by M. Hardy) or d) in a technical unit as in 36-G121 (Bodu et al 2014: 413, fig. 35, drawing by D. Molez). 


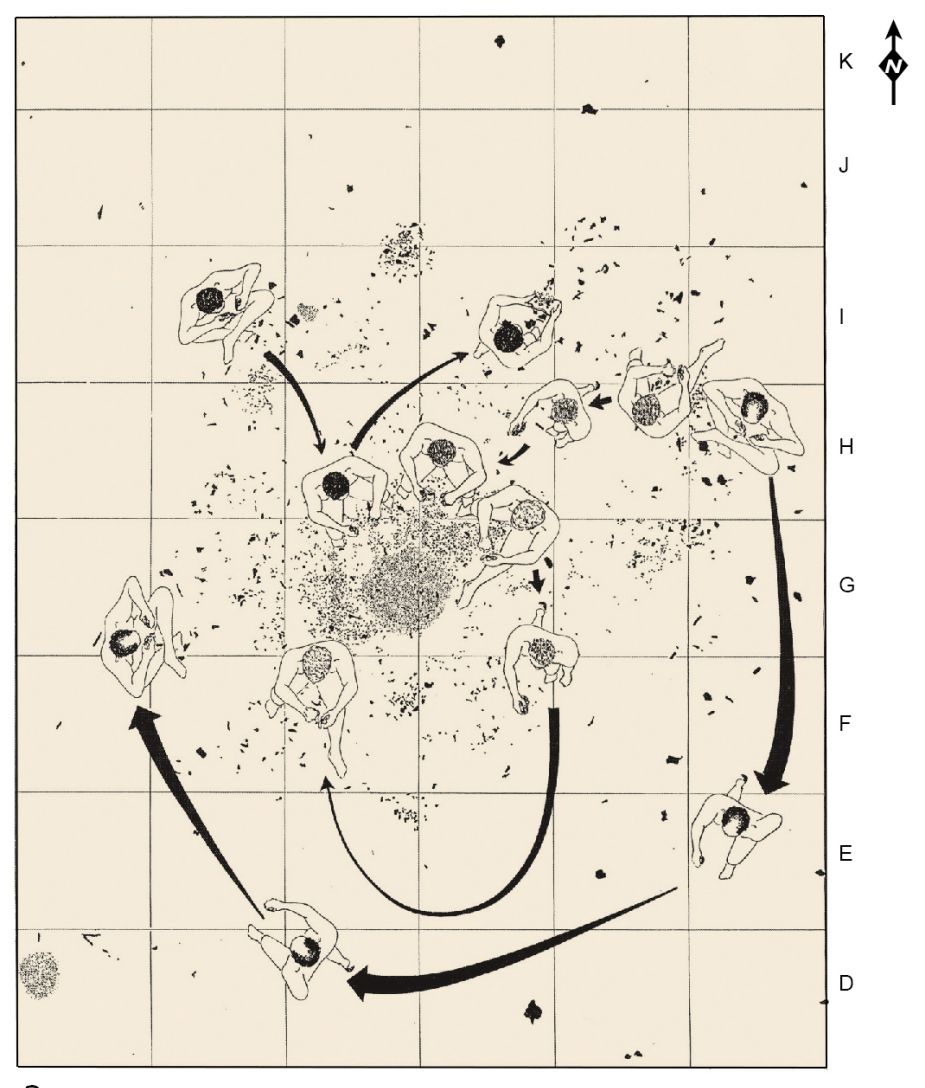

a

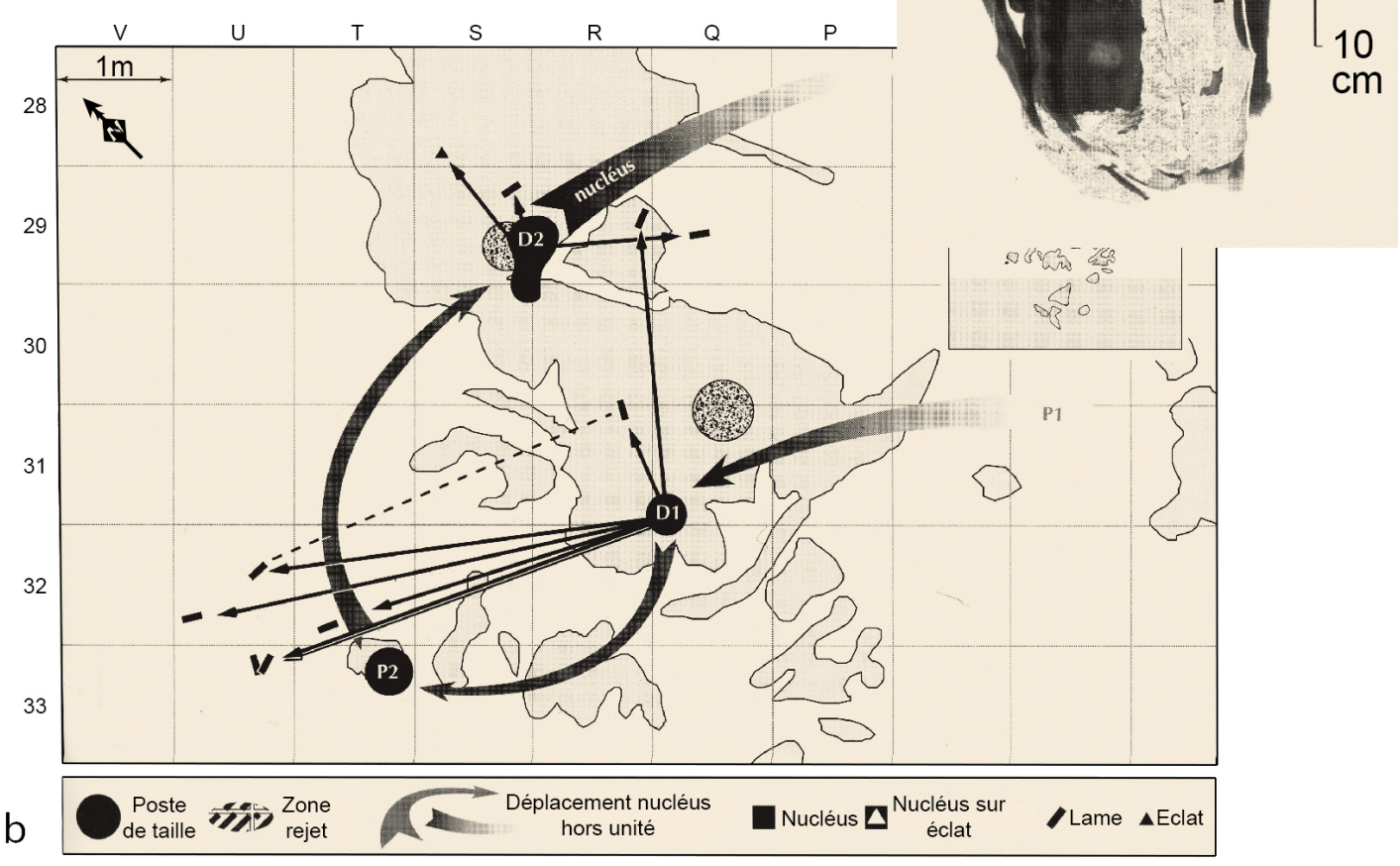

Figure 12. Déplacement des tailleurs au cours du travail : a) à Pincevent dans l'unité technique 36-G121, restitution des déplacements des quatre tailleurs qui ont travaillé là (Bodu et al. 2014: 413, fig. 35, dessin D. Molez) ; b) à Étiolles le bloc 328, taillé avec compétence, est préformé à l'extérieur de l'habitat S27/Q31 et débité dans l'atelier principal à l'est du foyer ; on constate un changement de lieu de taille à chaque rupture de phase de son exploitation (Pigeot 2004: 293, d'après M. Philippe et N. Pigeot).

Figure 12. Knappers moving during knapping: a) at Pincevent in technical unit 36-G121, reconstruction of the moving of the four knappers who worked there (Bodu et al. 2014: 413, fig. 35, drawing D. Molez); b) at Étiolles, block 28, knapped by a skilled knapper is first shaped out in the outside space of unit S27-Q31, then knapped in the main flint workshop, east of the hearth; there is a change of place at every change of knapping production phases (Pigeot 2004: 293, Drawn from M. Philippe and N. Pigeot). 

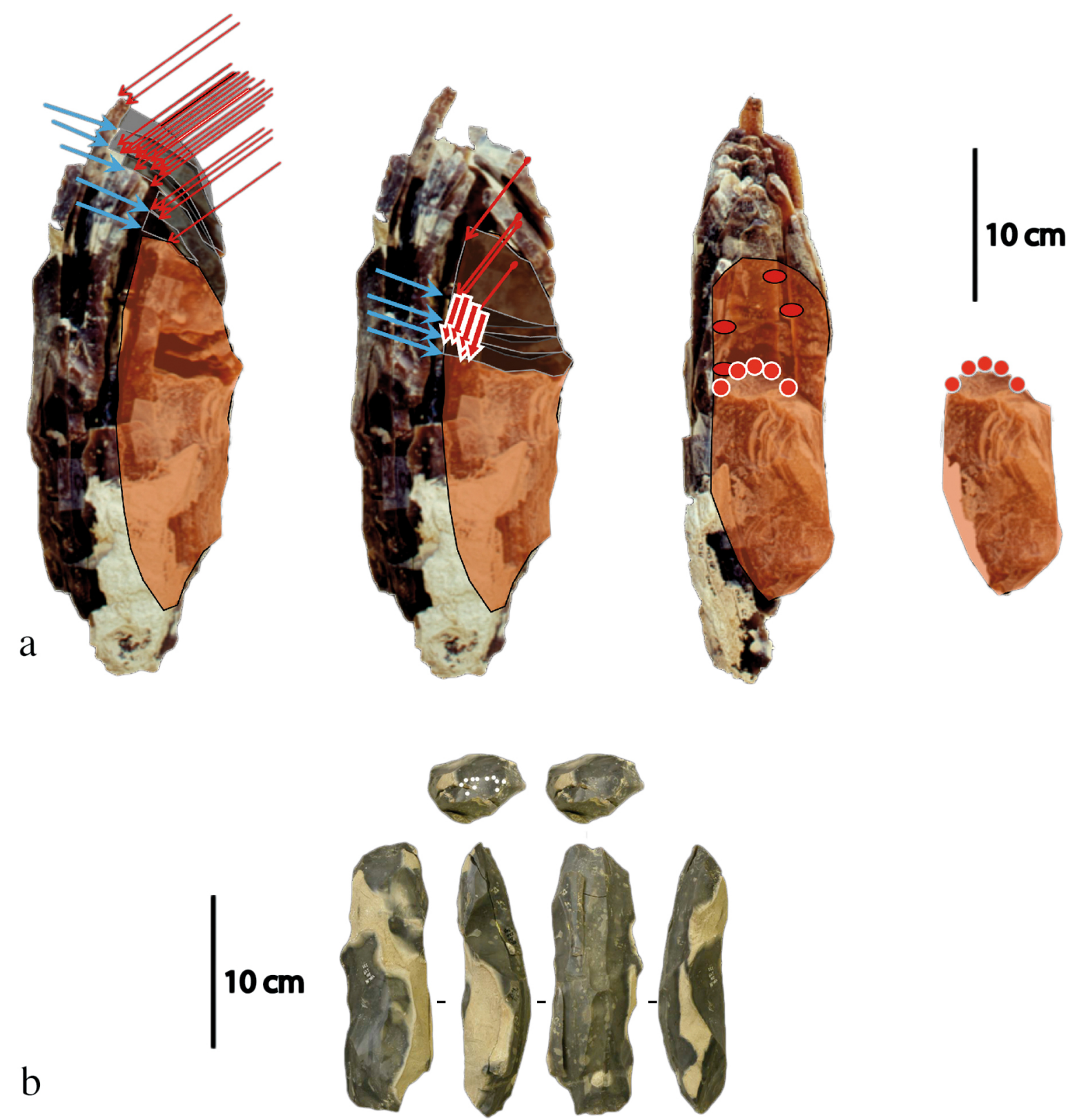

Figure 13. Au terme d'une séquence productive le nucléus abandonné par un tailleur de bon niveau peut être repris par un tailleur apprenti, ce qui se note par une rupture du niveau de compétence mis en œuvre. a) à Étiolles dans l'unité Q31 l, après avoir donné lieu à une belle séquence laminaire, le nucléus N14 est repris par un apprenti, reprise identifiée par une insistance infructueuse qui a donné lieu à des percussions visibles sur le plan de frappe (clichés N. Pigeot); b) sur cette séquence RL162 de Verberie on observe après une exploitation laminaire conduite par un tailleur très expérimenté, une reprise à la pierre par un tailleur inexpérimenté qui a porté plusieurs coups trop à l'intérieur du plan de frappe et en a finalement écrasé le bord (Rapport 2009: 41, fig. 43, clichés M. Langlais).

Figure 13. At the end of a productive sequence, an apprentice can get hold of a core discarded by a skilled knapper. This is evidenced by a change in skill level. a) at Étiolles in unit Q311, after yielding a high quality laminar sequence, the core N14 is knapped by an apprentice identified by an unsuccessful insistency that let visible traces of percussion on the striking platform (photos N. Pigeot); b) at Verberie, in sequence RL 162 that first yielded a laminar exploitation by a highly skilled knapper, an inexperienced knapper later made unskilled percussions with a hard stone hammer at the center of the striking platform and eventually crushed its edge (Rapport 2009: 41, fig. 43, photos by M. Langlais).

Enfin, cultures et sociétés manifestent leur spécificité à travers les techniques. Aussi étudier ces dernières conduit à s'interroger sur ce que les premières y inscrivent. Partant de l'objet et cherchant à remonter vers la pensée qui en est à l'origine, l'archéologue doit 
identifier les traces matérielles de ce sens, qu'elles soient directement et volontairement marquées dans les produits fabriqués ou qu'elles naissent de la convergence d'un faisceau d'informations. La richesse des données apportées par la technologie à partir d'une utilisation de la notion de chaîne opératoire a permis un progrès dans la connaissance des sociétés humaines. Ainsi en est-il à Pincevent d'un bloc travaillé dans l'unité 36-L114 du campement du niveau IV20 qui n'est ni une habitation ni un atelier. Cette séquence de débitage, proche du schème jusque dans les détails, signe l'extrême habileté du tailleur (Figure 14). Celui-ci s'installe à l'est du foyer, position qu'il avait déjà dans son habitation quasiment jointive, comme pour avoir sous son regard les tailleurs des autres résidences qui se sont eux installé pour travailler à l'ouest de leur foyer. Cette séquence semble avoir eu un rôle social, peut-être pour renforcer les liens entre les familles puisque les lames issues du plein débitage sont parties dans chacune des 4 résidences du campement où demeuraient des tailleurs tout à fait capables d'en produire de semblables (Julien \& Karlin 2014: 588). L’hypothèse émise est que son auteur qui semble, par de nombreux autres indices, avoir un statut particulier, occupait une position dominante et assurait la cohésion du groupe.

Ainsi à Pincevent en affinant l'utilisation du concept de chaîne opératoire, a-t-il été tenté de passer de la restitution des séquences de taille à une approche, timide il est vrai, de l'organisation sociale des Magdaléniens qui ont occupé le campement du niveau IV20: d'abord en distinguant trois groupes de tailleurs, les expérimentés, les compétents et les apprentis, puis en proposant un nombre de tailleurs identifiés par leurs productions lorsque cela paraissait possible dans chacune des quatre habitations, enfin en analysant les relations de ces tailleurs entre eux. Sans doute les préhistoriens sont-ils encore loin de la technologie culturelle telle que pratiquée par les ethnologues, mais ils tentent d'avancer à petits pas dans cette direction. On le voit, la chaîne opératoire n'est pas sortie toute faite du fameux paragraphe du Geste et la Parole. Elle a connu des développements progressifs, des fertilisations croisées dans lesquelles les ethnologues ont joué un rôle essentiel. Il a fallu plus que le concept créé par Leroi-Gourhan pour en faire la méthode analytique à large spectre qu'elle est aujourd'hui.

\section{Des avancées conceptuelles à des applications diversifiées}

\subsection{Un bilan d'étape : Le colloque de 1991}

À partir du moment où la chaîne opératoire, d'outil descriptif, est devenue un outil analytique (Perlès 1991a: 8), ses applications se sont diversifiées et ont été utilisées pour des problématiques extrêmement variées dans des contextes fort différents. On peut suivre la trajectoire de certaines de ces approches de leur création dans les années 80 à 90 jusqu’à aujourd'hui où elles continuent à irriguer la recherche en préhistoire.

Les XI ${ }^{\text {èmes }}$ Rencontres d'Archéologie et d'Histoire d'Antibes de 1990, « 25 ans d'Études Technologiques en Préhistoire » (Coll. 1991) sont un passionnant rapport d'étape qui montre le chemin déjà parcouru. L'archéologie expérimentale, les études actualistes, mais aussi les premières applications de la chaîne opératoire à des matériaux autres que la pierre y sont mises en valeur. Les progrès dont témoignent ces rencontres sont nombreux tant dans le domaine purement technique que dans l'élargissement des problématiques aux domaines socio-économique et culturel. Mais le malentendu avec certains préhistoriens étrangers qui expriment des critiques sur la démarche des préhistoriens français s’y lit déjà : leurs communications s'inscrivent bien dans le thème des rencontres mais restent dans le cadre théorique propre à leur communauté scientifique.

L'expérimentation, dont Bordes avait été l'initiateur et que Tixier transforme en une véritable démarche expérimentale est enrichie de protocoles d'expérimentation par Boëda, Pelegrin, Geneste, Texier (1984) (Figure 15). Les travaux de Pelegrin au Centre 
Archéologique et Historique de Lejre (Figure 16) à partir de 1975 (Pelegrin 1984) et un Cahier d'archéologie expérimentale créé par L’Archéodrôme de Beaune en 1985 en témoignent (Schmider et al. 1985).
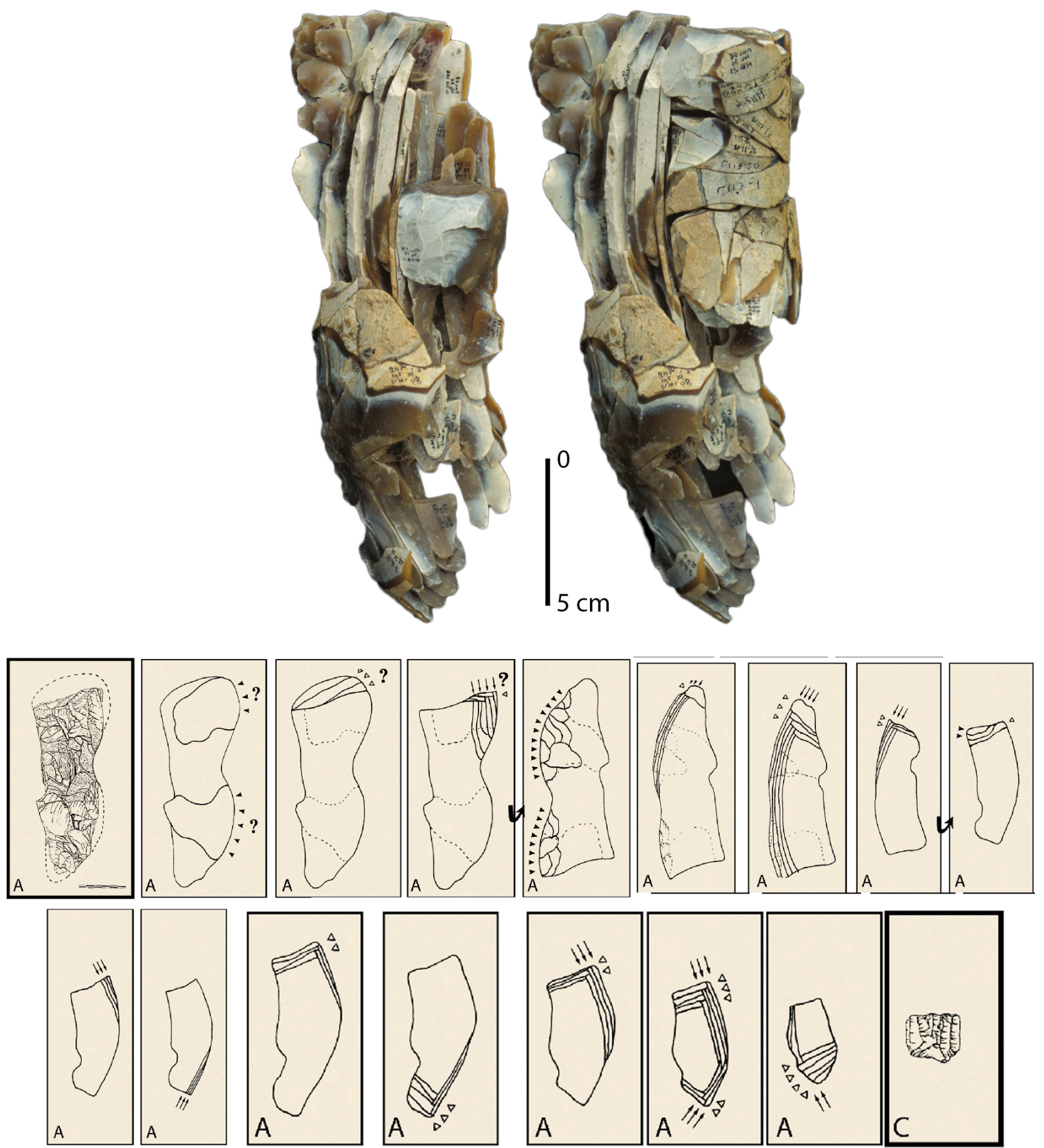

Figure 14. Réalisée dans l'unité 36-L115 du niveau IV20 de Pincevent, qui semble jouer un rôle social, la séquence de production M.118.1 relève d'un haut niveau de savoir-faire et ses lames qui ont circulé vers toutes les habitations pourraient avoir joué un rôle fédérateur dans le campement (Julien \& Karlin 2014: 394, fig. 22, cliché P. Bodu, dessin D. Molez).

Figure 14. The production sequence M118.1 knapped in unit 36-L115 of the Pincevent level IV20 that seems to play a social role, exhibits a high know-how level and its blades that have been distributed in all the domestic units may have played a unifying role in the camp (Julien and Karlin 2014: 394, fig. 22, photo P. Bodu, drawing by D. Molez). 


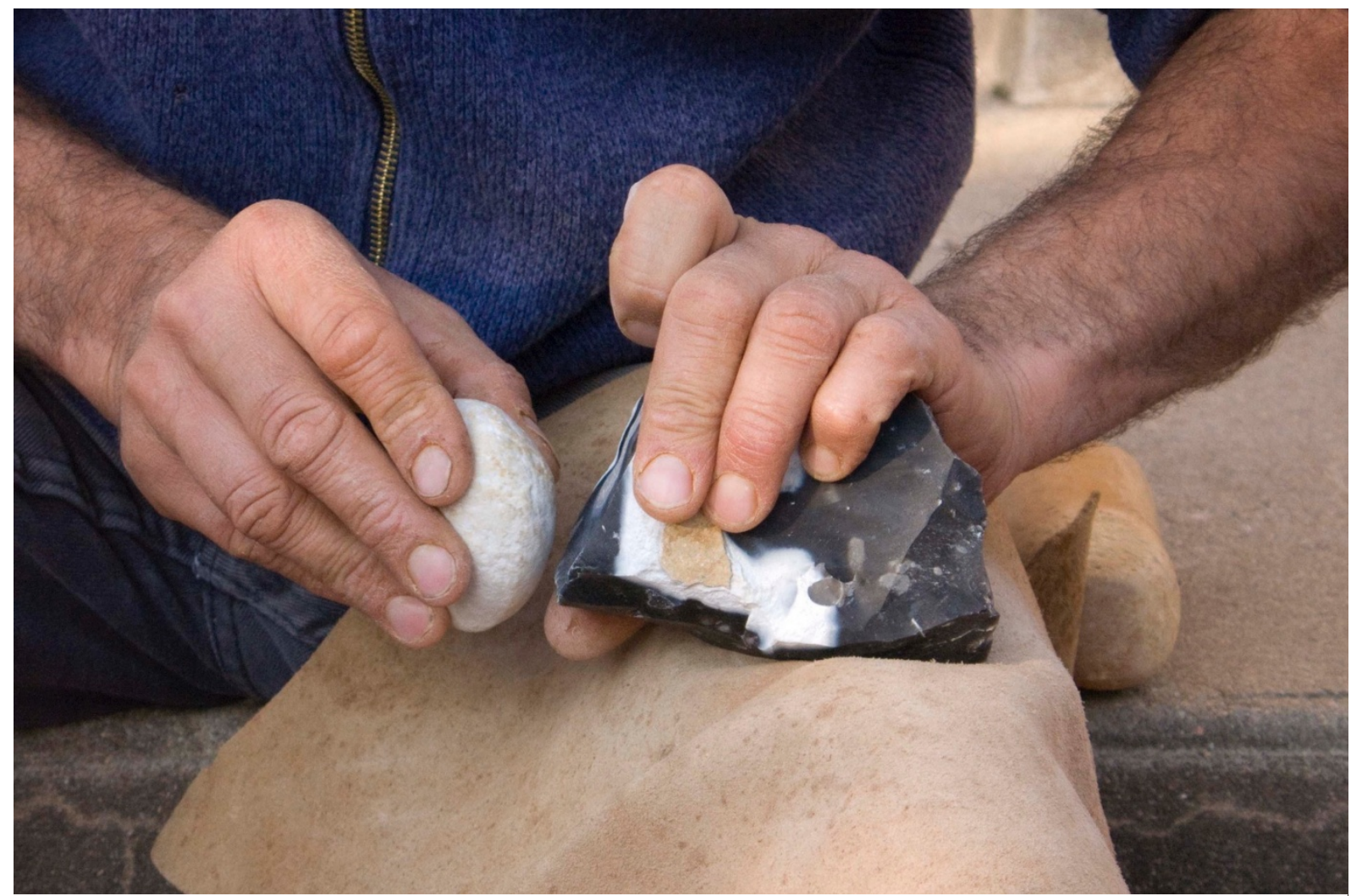

Figure 15. La taille expérimentale réalisée par des tailleurs modernes permet de tester la validité des hypothèses construites sur les méthodes de débitage à partir de l'observation des chaînes opératoires. Ici Pierre Bodu abrase la corniche d'un nucléus pour faciliter l'extraction des lames. Ce procédé, largement utilisé par les Magdaléniens de Pincevent, se déploie sur la face exploitée du nucléus, ce qui prolonge la vie du plan de frappe (cliché Équipe de fouille de Oisy - P. Bodu (Ed.)).

Figure 15. Experimental knapping realized by modern knappers allows to test the validity of hypotheses built upon the observation of chaînes opératoires on knapping methods. Here Pierre Bodu abrades the overhang of a core in order to more easily extract blades. This practice, widely used by Magdalenians at Pincevent, is spread on the core knapped front and this extends the striking platform duration (photo by Équipe de fouille de Oisy - P. Bodu (Ed.).

Dans le même temps, l'élargissement des applications se fait chronologique, du Paléolithique le plus ancien aux premières études actualistes. Il se fait aussi géographique avec des études sur des productions lithiques d'Israël, du Pérou, du Japon, d’Italie et d’Égypte, fondées sur des remontages dans les trois derniers cas. La chaîne opératoire commence aussi à s'appliquer à d'autres matériaux que le lithique, comme la céramique ancienne ou contemporaine (Holl 1985; Delneuf 1991). Un essai d'application aux primates n’aura pas de suite parce que les séquences de production sont trop courtes pour relever de ce type d'analyse (Beyries \& Joulian 1990).

À Bordeaux, Geneste ([Thèse 1985] 1988; 1991: 2) aborde la dimension régionale des systèmes de production lithique et montre que la chaîne opératoire permet la mise en évidence des comportements économiques impliqués dans l'acquisition des matières premières et dans leur distribution spatiale (Figure 17). Il est aussi un des premiers à réfléchir sur les systèmes techniques et à s’interroger sur la comparabilité des systèmes techniques préhistoriques et contemporains (Geneste 1990; 2010). Il s’appuie entre autres sur les concepts créés par Tixier et Inizan (Tixier et al. 1980; Tixier et al. 1984) ainsi que par Perlès (Perlès 1981).

À la même époque, Boëda introduit la notion de structure, «forme intégrant et hiérarchisant un ensemble de propriétés techniques qui aboutissent à une composition volumétrique définie (...) et caractérisée par l'ensemble des relations hiérarchiques et fonctionnelles des propriétés techniques »(1992: 40). C’est un état idéal situé entre les 
opérations d'initialisation et les opérations d'extraction de produits correspondants aux objectifs finaux : pièce bifaciale pour le façonnage, nucléus mis en forme pour le débitage.

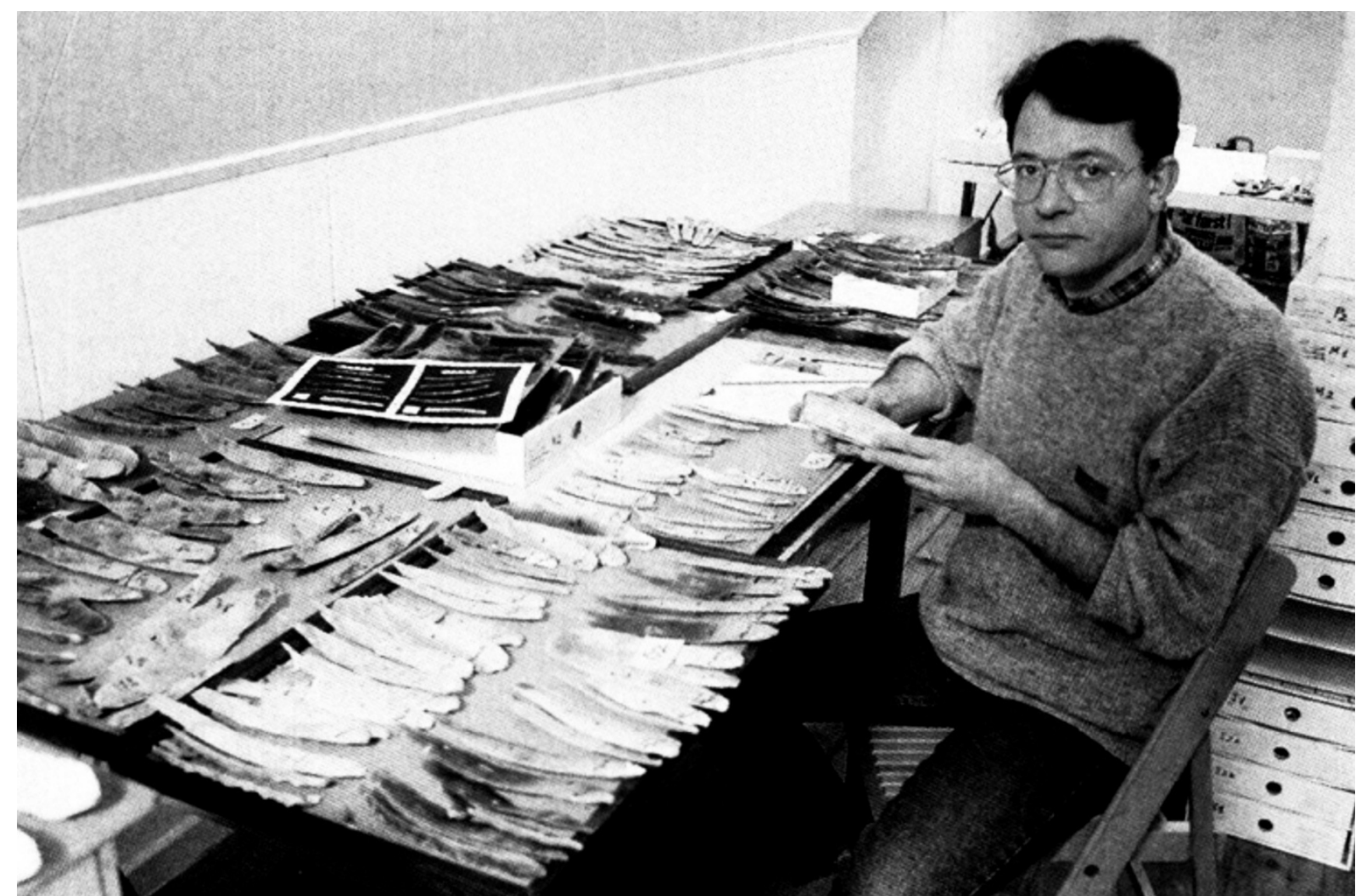

Figure 16. Jacques Pelegrin en 1993 au centre expérimental de Lejre (Danemark) cherche à résoudre les problèmes liés à la production de grandes lames (long-blades) : un excellent exemple d'expérimentation archéologique guidée par un protocole (Lejre Research Center, M. Rasmussen, 2001, cliché de O. Malling).

Figure 16. Jacques Pelegrin 1993 at the experimental center of Lejre (Denmark) works at solving the problem of long-blades production: an excellent example of an archaeological experiment controlled by a protocole (Lejre Research Center, M. Rasmussen, 2001, photo by O. Malling

Le problème de la représentation des séquences opératoires ainsi reconstituées se pose rapidement : représentations des blocs remontés, séquences individuelles, schèma opératoire représenté de façon synthétique ou structuré. Pour le Paléolithique supérieur, des dessins de blocs remontés sont publiés dès 1966 (Karlin 1966; 1972), en particulier grâce à la plume de R. Humbert dessinateur de l'équipe de Leroi-Gourhan (Figure 18a). Ils sont remplacés par des photos sur lesquelles les remontages sont souvent surlignés dans les publications d'Étiolles (Figure 18b). Le dessinateur M. Dauvois met lui au point un schéma diacritique où les conventions de dessin permettent de rendre compte d'une étape de remontage mais pas de l'ensemble de la chaîne (Dauvois 1976). Des représentations schématiques réduisant les blocs à des volumes simplifiés voient le jour à partir de 1990 (Boëda et al. 1990: 54, fig. 4 ; Reduron-Ballinger in Inizan et al. [1995] 1999: 132, fig. 54) (Figure 18c).

Les premiers graphes synthétisant le schème opératoire apparaissent à la fin des années 80. C'est d'abord une version simplifiée (Audouze et al. 1988: 81) qui se complexifie (Ploux et al. 1992) pour devenir un graphe sophistiqué à plusieurs entrées. Ainsi, Pigeot en fait une représentation très affinée lorsqu'elle différencie des moments variants, tactiques ou stratégiques (Pigeot 2004: 37, fig. 21) (Figure 19). 

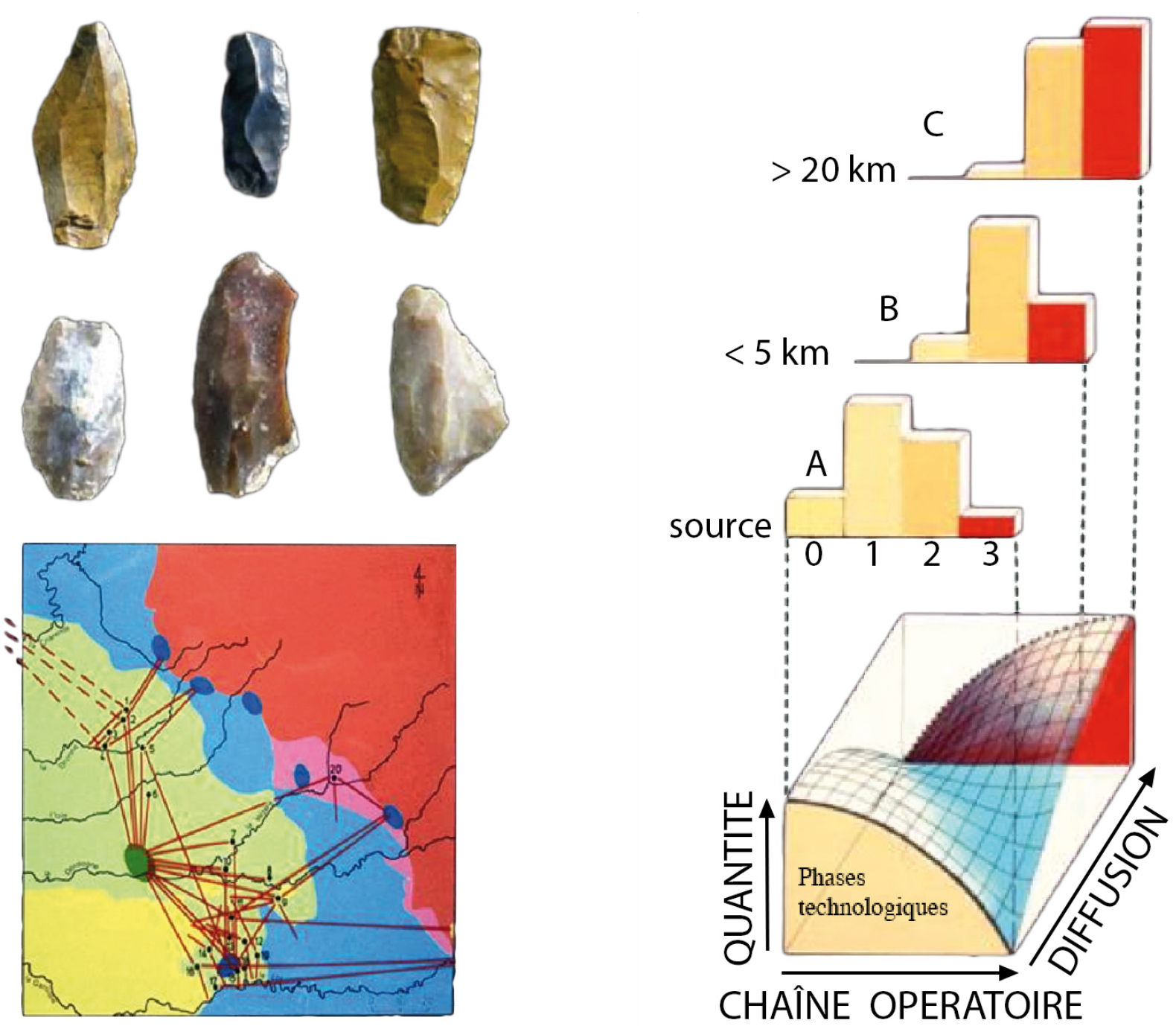

Figure 17. Rôle des facteurs technologiques et spatiaux dans l'interprétation économique de la distorsion observée dans la production lithique de type paléolithique sur la carte à gauche, avec au dessus des exemples de différents matériaux provenant de sources différentes, en graphique à droite. Plus les sources sont proches (A) plus les premières phases du débitage sont abondantes, plus elles sont lointaines (C) plus le rapport s'inverse et ce sont les supports et les retouches qui sont les plus nombreuses (phases technologiques : 1 produits corticaux ; 2 supports, éclats, lames, pointes et résidus - nucleus ; 3 retouches) (D’après Geneste 1991: 26, fig.13).

Figure 17. Role of technical and spatial factors in the economical interpretation of the discrepancy observed in the distribution of Paleolithic lithic productions, on the map on the right whith examples of different materials coming from different deposits, and with a diagram on the right. The closer the sources (A), the more the lithics coming from the knapping first phases are plentiful, the further away the sources, the more the relation reverses and the more blanks and retouched pieces are numerous (technical phases 1: cortical flakes; 2: blanks flakes, blades, points and residues - nucleus; 3 retouched pieces (Drawn from Geneste 1991: 26, fig.13).

\subsection{L'apport de la chaîne opératoire à l'analyse spatiale}

La dimension temporelle des séquences de production introduit une vision dynamique qui fut l'objectif premier de l'intérêt de Leroi-Gourhan pour les remontages. Mais elle n'est définitivement prise en compte dans l'analyse spatiale qu'à partir des années quatre-vingt. Initiée à Meer et Pincevent (Cahen et al. 1980), l'utilisation spatiale des résultats de remontages a d'abord été développée par les membres du laboratoire d'Ethnologie préhistorique responsables des fouilles de sites de plein air magdalénien dans le Bassin Parisien (Figure 20) et par les partenaires de leurs projets de recherche. (Cahen in Audouze et al. 1981; Audouze \& Cahen 1982; Audouze \& Cahen 1984; Pigeot [Thèse 1983] 1987; Olive 1985; 1988; Schmider \& Croisset 1990; Schmider 1992). 

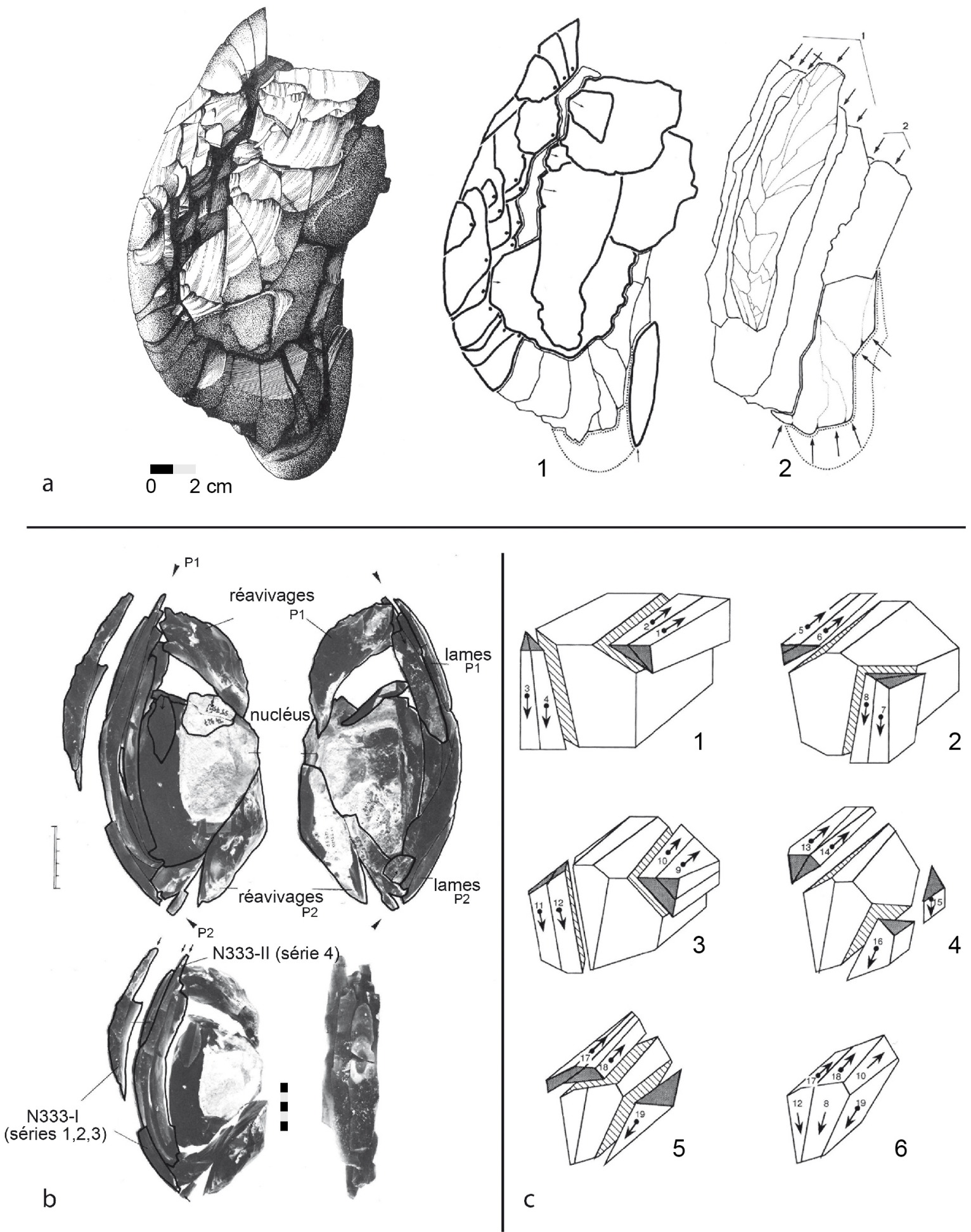

2
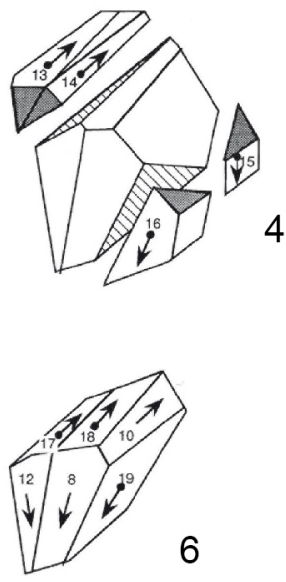

Figure 18. a) dessins de R. Humbert alliant une représentation réaliste en volume du remontage 17-C63 de Pincevent, niveau IV21, et une représentation analytique (Cahen et al.1980: 233-234, fig. 5-6) ; b) Surlignage sur photo du nucléus remonté N333 à Étiolles (Pigeot 1987 : pl. XXXVI) ; c) Représentation schématique d’une chaîne opératoire : de 1 à 5 obtention d'éclats épais et larges suivant des plans de débitage alternativement sécants et parallèles par M. Reduron-Ballinger (Inizan et al. [1995] 1999: 132, fig. 54).

Figure 18. a) drawings by R. Humbert combining a realistic 3D representation of the refittted block 17-C63 of Pincevent level IV21, and an analytical representation (Cahen et al.1980: 233-234, fig. 5-6); b) Highlighting on of the refitted block N333 of Étiolles on a photograh (Pigeot 1987: pl. XXXVI); c) schematic representation of a chaîne opératoire: from 1 to 5 extraction of thick and wide flakes along removal surfaces alternately secant and parallel by M. Reduron-Ballinger (Inizan et al. [1995] 1999: 132, fig. 54). 
DIVERS SEGMENTS

(temps, espace, etc.)
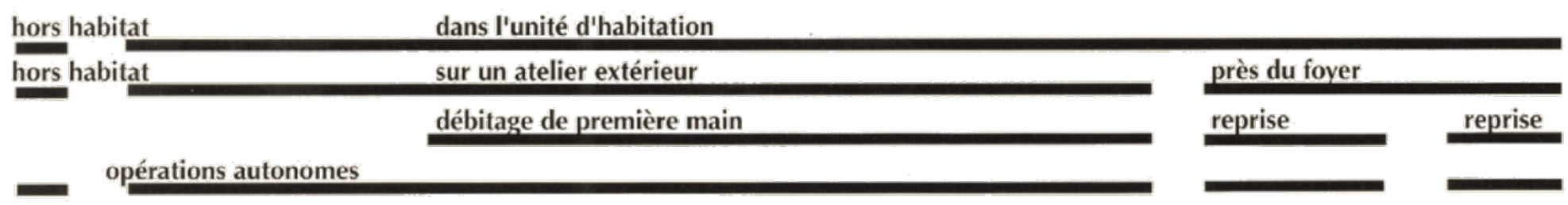

\begin{tabular}{|l|l}
\hline CHAÎNE TECHNIQUE & - \\
\hline PHASES & - \\
(SOUS-PHASES) & - \\
\hline SÉQUENCES & - \\
\hline (SOUS-SÉQUENCES) & - \\
\hline SÉRIES & - \\
\hline GESTES & - \\
\hline chaîne opératoire & \\
\hline moments variants & \\
\hline moments tactiques & - \\
\hline moments stratégiques & -
\end{tabular}

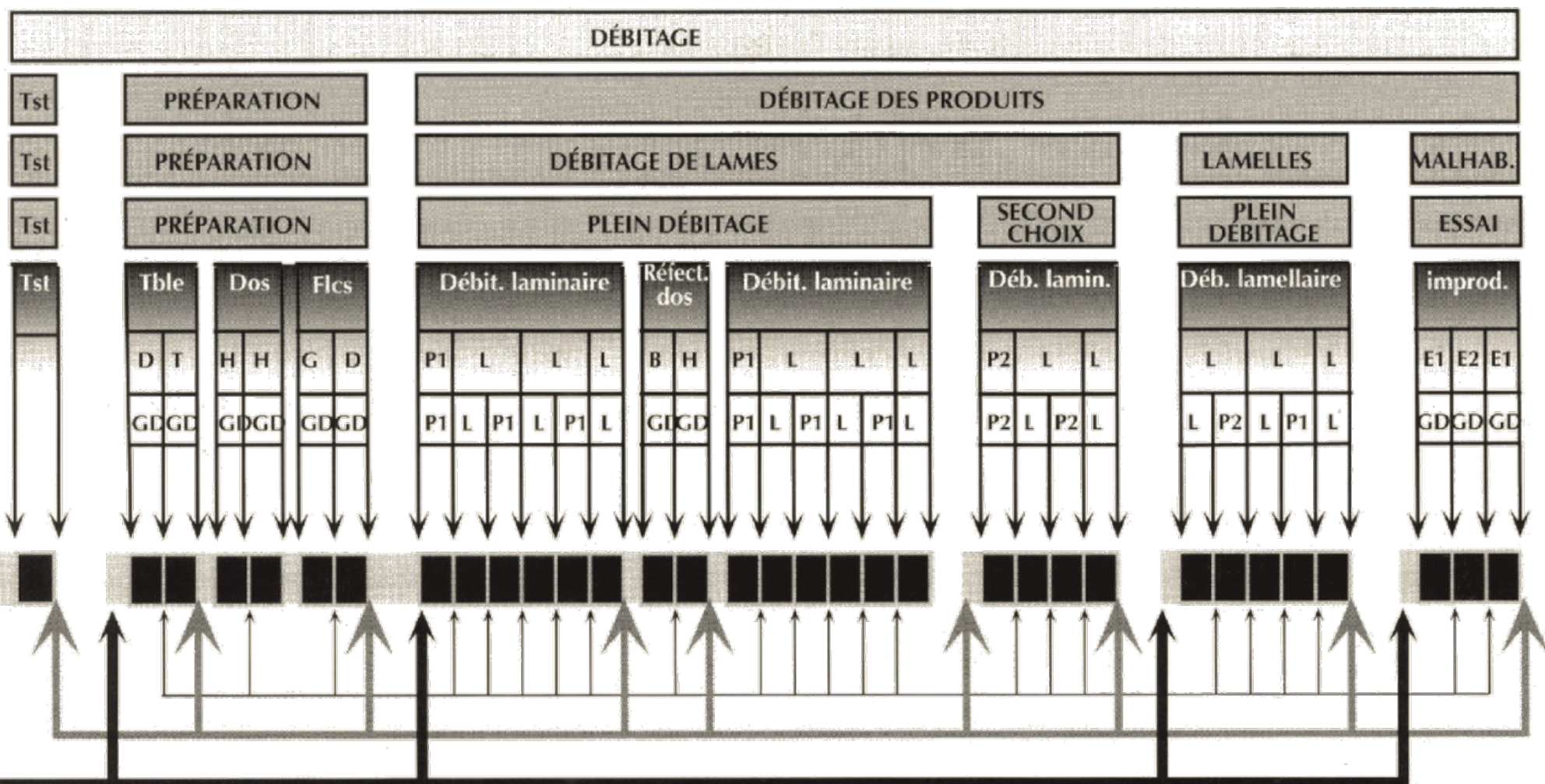

Figure 19. Complexification du découpage théorique d’une chaine opératoire de débitage en phases, en séquences, puis en gestes avec une évaluation de l’importance de ces étapes identifiées en moments variants, tactiques ou stratégiques. Pour les sous séquences : D, percuteur dur ; T, percuteur tendre ; H, haut ; B, bas ; G, gauche ; d, droit ; P1, premier plan de frappe ; L, débitage des lames ; P2, plan de frappe opposé ; E, débitage de mauvaises lames (Pigeot 2004: 37, fig. 21).

Figure 19. A more complex representation of the theoretical distribution of a chaîne opératoire in phases, sequences, and in gestures, introducing an evaluation of these different stages identified as variant, tactical or strategic moments. D, hard stone hammer; T, organic hammer: H, top; B, bottom; G, left; d, right; P1, fist striking platform; L, blades extraction; P2 striking platform opposed to P1, E, extractions of low quality blades (Pigeot 2004: 37, fig. 21). 


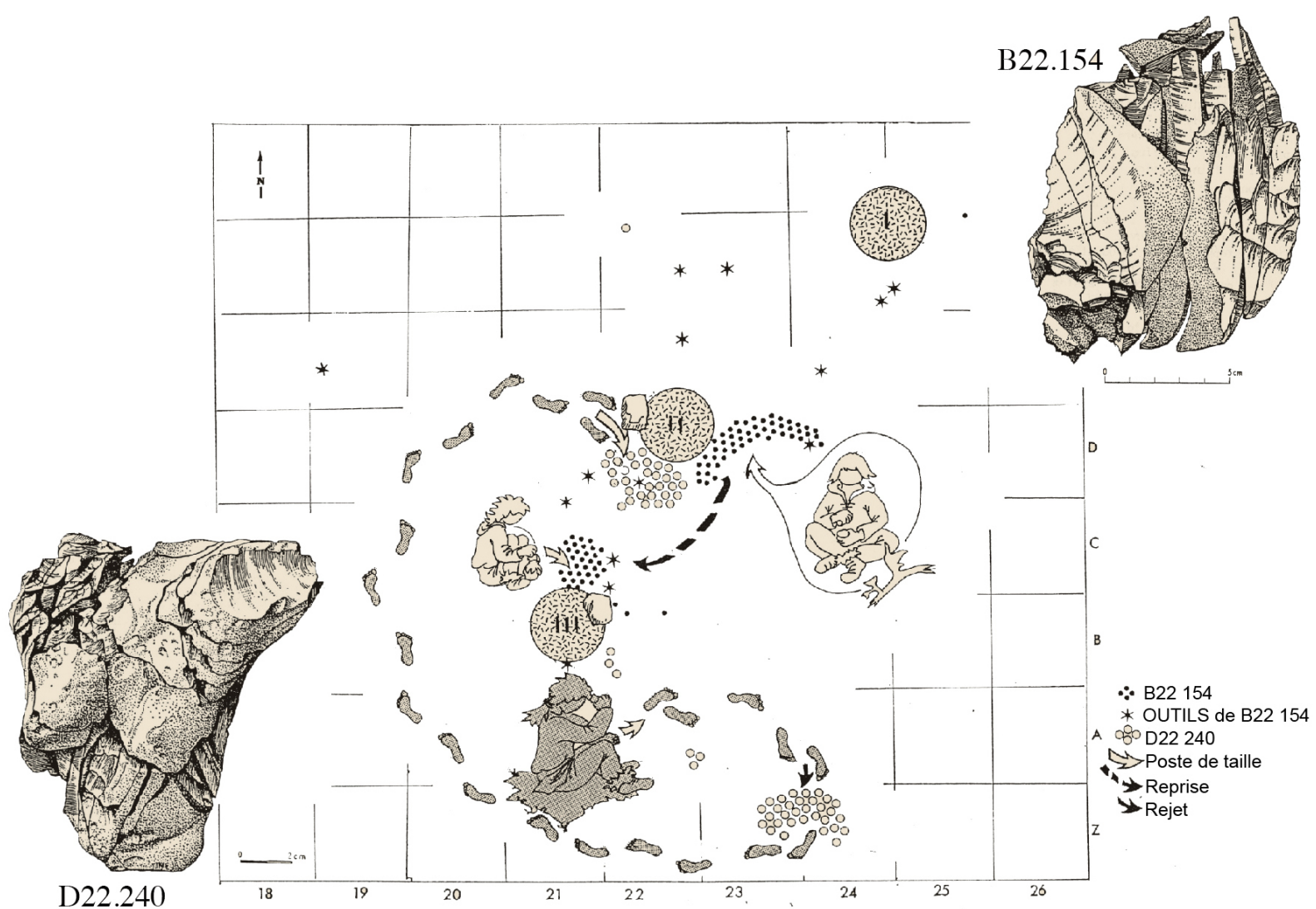

Figure 20. Organisation dans l'espace de deux remontages de l'Habitation $n^{\circ} 1$ de Pincevent. La séquence D22.240 est mise en forme à quelques distances du foyer III ; les produits de cette importante mise en forme sont rejetés dans un amas extérieur ; le plein débitage se poursuit sur les bords du foyer II. C’est la même main qui semble avoir réalisé l'ensemble de l'exploitation et le tailleur a donc préparé son bloc de manière à ne pas polluer l'espace de vie puis s'est installé au poste de taille principal pour le plein débitage laminaire. L'exploitation de la séquence B22.154 se déroule près du foyer II puis se termine aux abords du foyer III. Le tailleur installé à côté du poste principal sur le bord du foyer II produit des supports laminaires dont beaucoup sont transformés en outils. Lorsqu'il abandonne le nucléus il estime la séquence terminée ; le nucléus est repris par une main inexpérimentée qui va s'exercer aux abords du foyer III devant le poste permanent et le défigurer rapidement (Dessins nucléus R. Humbert, dessin plan C. Karlin).

Figure 20. Spatial distribution of pieces coming from two refitted blocks in Pincevent Habitation $\mathrm{n}^{\circ} 1$. The shaping out of sequence D22.240 takes place at a short distance from Hearth III; the refuse from this important shaping out is discarded in an outside concentration; then, the laminar debitage takes place on the edge of the hearth. A same knapper has done the whole of the knapping. He prepared his block away to avoid polluting the domestic space then moved to the main knapping station for the blades extraction. The knapping of the sequence B22.154 takes mostly place near hearth II and ends near hearth III. The knapper working close to the main knapping spot extracts laminar blanks - many of which are transformed in tools. He considers the core as exhausted; an inexperienced knapper takes over, and go and exercise near hearth III; he quickly ruins the core (Drawings refitted blocks R. Humbert, drawing map by C. Karlin).

Elle amène à poser le problème suivant : comment représenter la dispersion spatiale des produits d'une même séquence opératoire (Figure 21a) ? Dans un premier temps cela donne lieu à de véritables toiles d'araignée difficiles à comprendre : le nucléus sert de pôle auquel sont directement raccordés les produits, indépendamment de la chronologie de la séquence. Puis ces lignes tentent de suivre la chronologie des enlèvements (Cziesla 1987). Dès 1983 Pigeot s'interroge : linéaire, dans l'ordre de débitage, ou synthétique (Pigeot [Thèse 1983] 1987: 8), mouvement individuel ou collectif des silex (Olive 1988) (Figure 21b) ? C'est lorsque sont pris en compte à la fois la chronologie, la nature des enlèvements regroupés en phases (aménagements, remises en forme, travail sur les plans de frappe, produits recherchés de plein débitage, etc.) que la représentation graphique devient lisible pour le lecteur. 


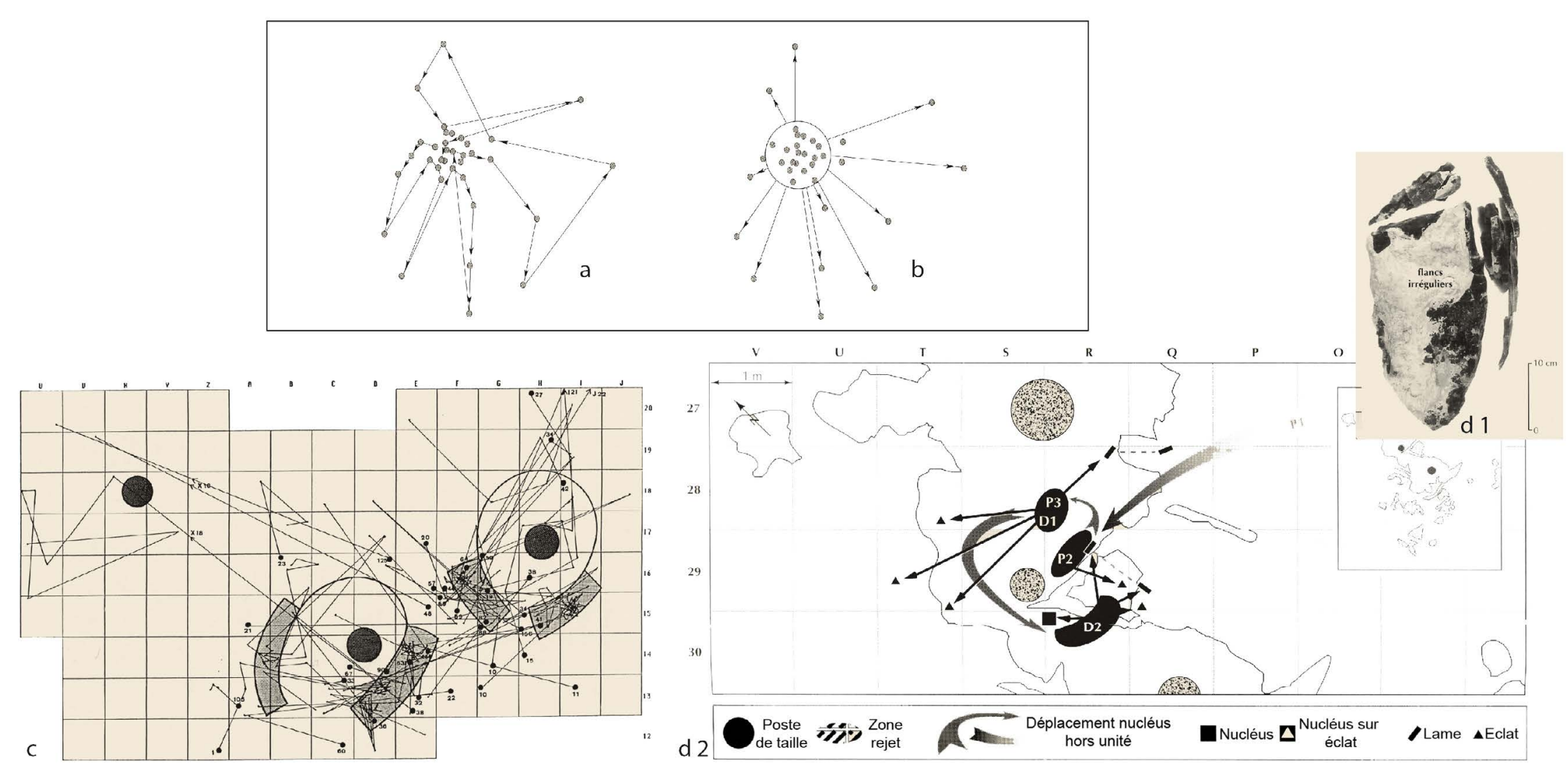

Figure 21. Comment représenter les remontages : a) en suivant l'ordre chronologique des enlèvements ou b) en montrant le mouvement des objets à partir du poste de débitage (Cattin. 2002: 159, fig. 217, DAO Marielle Zanetta, Laténium) ? c) Cet autre exemple de liaison voulant restituer un ordre chronologique ne permet que de lire la circulation entre les deux unités de Marsangy (Schmider \& Croisset 1990: 435, fig. 3) ; d) ou en reliant entre elles des phases différentes d'une même séquence, et non chaque produit, ce qui simplifie la représentation : le début de la préparation du nucléus 341 d’Étiolles s'est effectué hors de l'unité, se poursuit (P2) sur le bord de la nappe centrale en R29 et s'achève (P3) à moins d'un mètre de là en S28, ce qui peut correspondre au simple retournement du tailleur ; le débitage laminaire (D1) est entamé sur ce poste puis le nucléus est rapproché du secteur Q31 pour y être repris (D2), et le nucléus est abandonné sur place (Pigeot 2004: 295, d’après M. Philippe \& N. Pigeot).

Figure 21. How to illustrate refitting: a) following the order of removals or b) drawing the moves of pieces from the knapping station (Cattin. 2002: 159, fig. 217, DAO Marielle Zanetta, Laténium) ? c) using the chronological order as in this example wich only gives evidence of a circulation between two units at Marsangy (Schmider \& Croisset 1990: 435, fig. 3); d) or simplifying the representations in drawing relations between the various phases and not between pieces. The flint block 341 at Étiolles was shaped out outside the habitation unit, then was moved to the central scatter in R29 (P2) in S28, and ended less than 1 m away (P3). This may be the consequence of the knapper turning around. The laminar debitage (D1) starts there, then the core is brought near unit Q31 for a second laminar sequence (D2) and the core is left there (Pigeot 2004: 295, Drawn from M. Philippe and N. Pigeot). 
À Pincevent, grâce à des remontages à longues distance, P. Bodu relie les différentes occupations du niveau IV20 les unes aux autres (Bodu 1993) et ouvre l'analyse spatiale aux relations entre les différentes unités identifiées, résidences ou ateliers, dont la contemporanéité a été démontrée par les observations géomorphologiques et les coupes au latex (Orliac 1975; 1989). À Étiolles dans le niveau des secteurs Q31 et S27, l'exploitation des nombreuses chaînes opératoires reconstituées (21\% des silex ont pu être remontés) va plus loin dans la spatialisation des séquences de débitage : elle introduit leur succession dans le temps et l'espace pour aboutir à une chronologie des occupations des secteurs et des groupes de tailleurs (Pigeot 2004).

Parallèlement, des méthodes nouvelles d'analyse spatiale sont développées et testées sur les données de Pincevent et de Verberie. Le premier à avoir tenté une analyse spatiale quantitative sur un site magdalénien du Bassin parisien a été A. Hesse sur les Tarterets (Hesse 1971). La section 36 du niveau IV20 fait l'objet de plusieurs essais : I. Johnson (Johnson 1976; 1984) propose une méthode fondée sur les densités locales, K.W. Kintigh et A.J. Ammerman une méthode fondée sur la classification des coordonnées (Kintigh et Ammerman 1982). D. Stapert applique sa méthode des Rings and Sectors à Pincevent et Verberie (Stapert 1990; 1992). F. Djindjian utilise l'analyse des correspondances dont les résultats lui semblent meilleurs que ceux des travaux statistiques antérieurs (Djindjian 1990). Mais il s'agit toujours d'un rendu statique, appauvri par rapport à la richesse d'informations des chaînes opératoires spatialisées et le résultat de Stapert, par exemple, est contredit par l'analyse fine des postes de travail autour du foyer à Verberie (Audouze 2010: 150-152).

\section{Cinquante ans plus tard}

Depuis qu'en 1965, l'analyse des premiers remontages systématiques de l'Habitation n 1 (Karlin in Leroi-Gourhan \& Brézillon 1966) a initié le transfert du concept "chaîne opératoire " des ethnologues vers les préhistoriens, un peu plus de cinquante ans se sont écoulés. Où en sommes-nous aujourd'hui ?

La plupart des préhistoriens français incluent désormais un spécialiste du lithique dans leurs projets. La chaîne opératoire, comme outil analytique, fait partie intégrante de l'étude des sites ou des collections préhistoriques. «En France aujourd'hui, il n'est pas jusqu'au moindre rapport de diagnostique en préhistoire qui ne contienne quelques lignes traduisant une volonté de caractérisation technologique minimale » (Valentin 2007: 238). Il existe des différences méthodologiques selon qu'on travaille sur les périodes du Paléolithique ancien et moyen pour lesquels l'identification des formes volumétriques prédomine ou sur les époques postérieures, et selon qu'il est possible de procéder ou non à des remontages. Le remontage mental est prédominant pour les périodes les plus anciennes en raison de l'impossibilité de disposer de séquences de débitage complètes. Il l'est également pour la préhistoire récente extra-métropolitaine, des îles du Pacifique par exemple (Forestier 1996; Hermann 2013) et dans tous les cas où la durée d'occupation, la quantité de matériel à étudier ou l'absence de chaînes de production complètes les rendent impossibles.

Une nouvelle phase est marquée par l'apport des travaux ethnoarchéologiques qui enrichissent les interprétations fondées sur les analyses des chaînes opératoires et de leur variabilité (Beyries 2002; 2008; Beyries et al. 2002; Beyries \& Rots 2006; 2008). Cette approche trouve une concrétisation dans la publication du grand campement de Pincevent où ces résultats, projetés sur les plans du IV20, restituent les relations sociales au sein d'un campement de plusieurs groupements familiaux magdaléniens dont les membres tailleurs ont été identifiés par leur production (Julien \& Karlin 2014) (Figure 22). 


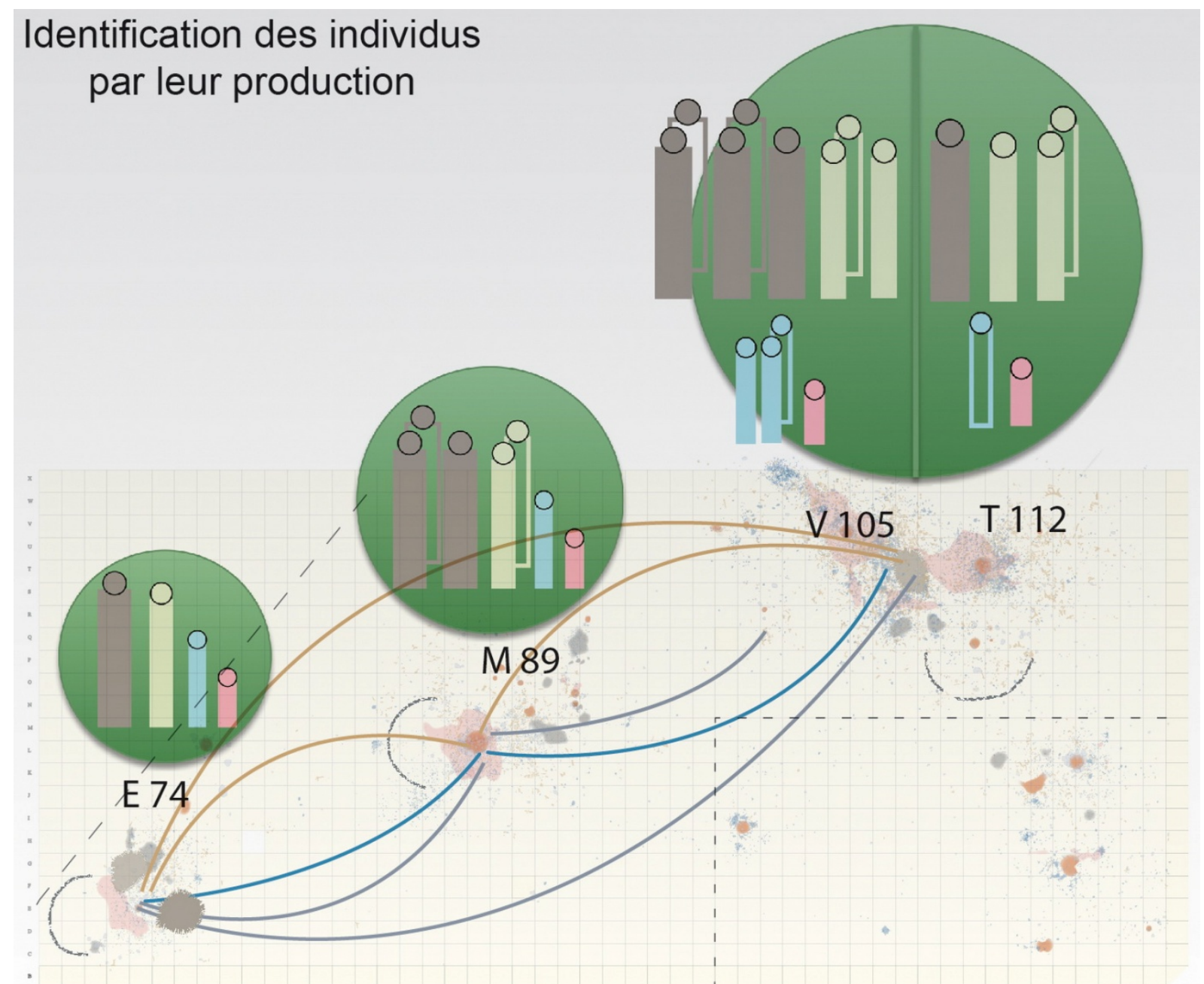

Figure 22. L'analyse des séquences de chaînes opératoires reconstituées a permis d'identifier un certain nombre de tailleurs dans chacune des quatre unités de résidence du campement IV20 de Pincevent. À ces individus reconnus et différenciés par la qualité de leur production ont été ajouté arbitrairement un nombre d’individus n’ayant pas taillé afin de proposer une évaluation de la composition de chaque unité sociale (les silhouettes brunes sont les hommes, les jaunes les femmes, les bleus les adolescents, les roses les enfants ; les individus ajoutés en fonction de la masse de vestiges dans l'unité sont en contours). Les remontages permettent ensuite de suggérer l'existence de deux groupes d'importance différente. Des liens forts semblent exister en raison de remontages très nombreux des différentes catégories de vestiges entre l'unité V105, le groupe le plus nombreux, et l'unité T112, avec un statut dominant à ce dernier. Un même statut dominant, mais à un moindre degré, pourrait être conféré à l'unité M89 par rapport à l'unité E74, visible en particulier dans la circulation des produits de silex. Enfin il apparait une position d'allégeance du deuxième groupe de familles par rapport à la cellule T112 (Julien et al. 2014: 357-368, DAO M. Hardy).

Figure 22. The analysis of reconstructed sequences of chaînes opératoires leads to the identification of a number of individuals who differ by the quality of their production in each of the four domestic units of level IV20 at Pincevent. A number of non-knapping people were arbitrarily added to these individuals in order to propose an evaluation of the composition of every social unit (brown silhouettes are men; yellow for women, blue for teenagers, pink for children. Non-knapping people added according to the volume of artefacts per unit are outlined). The refits allow to hypothesize the existence of two groups of different importance. Based upon the numerous refits, tight relations seem to exist between unit V105 (the most numerous group) and unit T110, which has a dominant status. A same dominant status, but to a lesser degree, may be assigned to unit M89 in relation to unit E74, visible in the circulation of flint products. Last, the second group seems to be in position of allegiance to the unit T112 (Julien et al. 2014: 357-368, DAO M. Hardy). 
Dans le domaine expérimental, la construction de référentiels expérimentaux et ethnoarchéologiques se poursuit (notamment Beyries 2008; Bril et al. 2012) et une volonté de rigueur s'exprime dans des protocoles prédéfinis (Brenet et al. 2009; Pétillon et al. 2011). Pelegrin mène une action pédagogique en affinant la description des stigmates caractéristiques des différentes techniques de taille à partir de l'observation de produits laminaires obtenus expérimentalement (Pelegrin 2000; 2010; 2012; Pelegrin \& Yamanaka 2007; 2012; 2016). Un projet mené par V. Roux prévoit de donner accès en ligne aux collections expérimentales et ethnoarchéologiques du laboratoire CNRS Préhistoire et Technologie (Labex « Passés dans le présent » 2013-2016).

Dans le domaine du débitage par pression que Tixier a largement défriché à la suite de Crabtree, Inizan a continué à jouer un rôle majeur souligné par la session du Congrès de Lisbonne de 2006 sur le débitage par pression donné en son honneur et celui de Tixier. L'article qu'elle a publié dans les actes (Desrosier 2012: 11-42; Pelegrin 2016) met en lumière les résultats des vingt et quelques années qu'elle a consacré à identifier cette technique en Afrique du Nord, et du Proche-Orient à la Sibérie et le Japon en passant par le Moyen-Orient, l'Asie Centrale et orientale.

L'adaptation de la chaîne opératoire comme outil analytique à de nouveaux matériaux conduit à des développements méthodologiques et techniques et à la construction de référentiels adaptés : notamment sur l'os, le bois de cervidé (Figure 23) et l'ivoire façonnés, dont la variabilité biologique interindividuelle complique l'observation des stigmates anthropiques et où chaque étape du travail tend à oblitérer les traces des étapes antérieures (Averbouh 2000; 2014). La chaîne opératoire y est partie prenante d'études technoéconomiques (par exemple, David 2004a; 2004b, Tartar et al. 2006; Ledosseur 2006; Tejero \& Christensen 2011; Müller \& Reiche 2011; Manca 2013; 2016; Marquebielle 2014; Christensen 2015; Averbouh et al. 2016; Goutas \& Tejero 2016). Les travaux de terminologie multilingue du Groupe de Recherche Européen GDRE PREHISTOS (Averbouh et al. 2010; Ledosseur 2014) occupent une place particulière en raison de la participation de nombreux chercheurs européens (Averbouh 2016). D’autres développements ont été faits pour la céramique (Roux 2013a), pour le cuir (Beyries \& Rots 2010), le bois (Alix 2001) ou encore les coquillages (Vanhaeren 2002; Rigaud 2011), et le travail de la pierre dans la fabrication des perles (Bril \& Roux 1993). Cet élargissement s'étend à l'Âge du Bronze (Ghesquière et al.1997; Bailly 2002) ; à la préhistoire récente de Patagonie (Legoupil \& Pigeot 2009) ou des îles du Pacifique (Hermann 2013) pour le lithique; à la protohistoire et aux périodes historiques en Europe et en Afrique pour la céramique (Manem 2010) et le métal (Fluzin 2002; Fluzin et al. 2011). Au-delà d'une application à des techniques de transformation des matériaux, la chaîne opératoire est adoptée pour analyser des faits économiques ou sociaux (Roux \& Matarasso 1999). Ainsi les archéozoologues travaillant sur la gestion des ressources animales font référence aux cadres théoriques développés par la technologie française (Horard-Herbin \& Vigne 2005), tandis que les anthropologues physiques utilisent la chaîne opératoire pour analyser le déroulement des rituels funéraires (Noret 2012; Valentin et al. 2014). Les analyses physico-chimiques non destructives (Reiche et al. 2011; Large et al. 2011) accompagnent cette évolution ; de même que l'imagerie numérique avec représentation 3D (Legrand-Pineau \& Sidera 2014) présente par exemple dans beaucoup des interventions de jeunes chercheurs au workshop The Big Puzzle 30 years after (Mai 2017, Tarragona) à paraître dans un numéro de la revue Archaeological and Anthropological Sciences. 

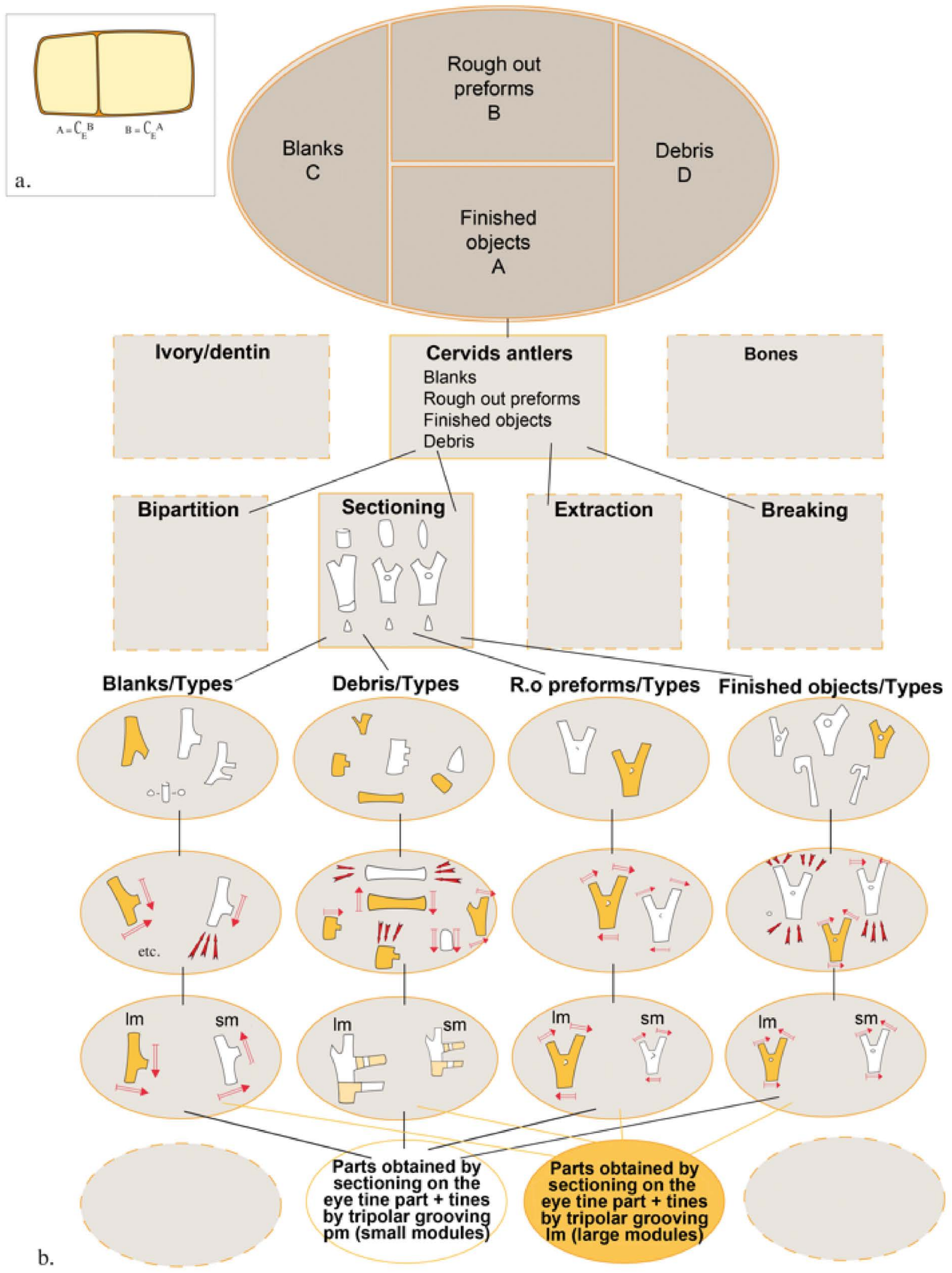

Figure 23. Remontage par défaut des bois de cervidé : représentation schématique du matériel d'étude et de la constitution de sous-ensembles complémentaires : exemple d'une répartition sur six niveaux aboutissant à la constitution de sous-ensembles technologiquement homogènes témoignant de la production de bâtons percés (Averbouh 2000: fig. 17).

Figure 23. Default refitting of cervid antlers: schematized representation of the material under study, and the making of complementary subsets: example of a distribution in six levels resulting in creating technically homogenous subsets accounting for the making of straighteners (Averbouh 2000: fig. 17). 
L'identification de niveaux d'habileté différents au sein d'assemblages lithiques (Ploux 1989) a ouvert tout un champ de recherche sur l'apprentissage de la taille (Roux 1991; Pelegrin 1991b; Roux \& Bril 2002; Roux \& David 2005). Ces travaux ont d'abord été l'apanage des collaborateurs de Leroi-Gourhan et de Tixier. Ils ont mis du temps à se propager au delà de ce cercle, en particulier vers le Sud-Ouest de la France (Simonet 2012; Klaric sous presse; Bordes \& Bachellerie sous presse) (Figure 24), alors qu'ils ont démarré beaucoup plus tôt en Europe du Nord, notamment au Centre Archéologique et Historique de Lejre (Fischer 1989; 1990) où ils ont fait l'objet d'une table-ronde réunissant surtout des archéologues d'Europe du Nord et des Britanniques en 2008 (Eigeland \& Sternke 2011). L'extension de ce thème de recherche se fait aussi dans le temps, avec une avancée vers le Paléolithique moyen (Stapert 2007) et même le Paléolithique ancien récent avec les maîtres et l'apprenti tailleurs de biface de Boxgrove (Leroyer 2016; Leroyer sous presse a; Leroyer sous presse b) (Figure 25).

Ces niveaux d'habileté ont été interprétés en termes de compétences cognitives (Pigeot 1991; 2011; Pelegrin 2005) et psycho-motrices (Roux 1997). Ils donnent lieu à une importante littérature, tant dans le domaine de la céramique avec en particulier les conditions d'usage du tour de potier (Roux \& Corbetta 1990; Roux 2011; Roux \& Courty 2016) que dans des recherches sur l'évolution des capacités cognitives au cours de l'évolution (Roux \& Bril 2005; Roux 2013a).

S’en sont suivies aussi des recherches sur la spécialisation (Pélegrin 2007), et la division sexuelle des tâches où la présence des femmes est d'abord évoquée à titre d'hypothèse (Ploux 1989; Chauchat \& Pelegrin 1994; Julien \& Karlin 2007) puis analysée (Audouze 2010; Julien \& Karlin 2014: 587-588).

L'innovation technique durant la préhistoire est un champ disciplinaire souvent évoqué dans des articles divers mais peu l'ont pris comme sujet d'analyse ; on y trouve l'influence des ethnologues français et anglo-saxon (van der Leeuw 1994; van der Leeuw \& Torrence 1989; Roux 2013b).

\section{Qualitatif ou quantitatif ?}

On a reproché l'absence de quantification dans les travaux utilisant la chaîne opératoire : " As several scholars have pointed out (...), the chaîne opératoire approach can be highly subjective if a purely qualitative, systemic approach is used. In order to make this study as objective as possible, we incorporate various quantitative parameters » (Wadley et al. 2016: 137; Bar Yosef \& Van Peer 2009; Tostevin et al. 2011; Djindjian 2013). Mais que quantifier et pourquoi ? Les lithiciens français n’ont pas vu d'intérêt à décompter et mesurer tous les enlèvements issus d'un débitage ou d'un façonnage dans la mesure où il leur semblait que cela mettait sur un même plan des éléments de nature différente et qui, dans la pensée même du tailleur, ne pouvaient avoir la même valeur, par exemple des éclats de mise en forme non utilisables et non utilisés et des lames de plein débitage destinées à être utilisées brutes ou aménagées en outils. C’est donc à un stade ultérieur qu'ils ont introduit des décomptes et la mensuration de certaines pièces dans quelques uns de leurs travaux : après que l'identification du ou des schèmes opératoires caractéristiques d'un niveau archéologique, d'une occupation, ou d'un territoire à un moment donné, ait permis de diviser les séquences de production qui s'y rattachent en sous-séquences pertinentes par rapport au problème posé (par exemple Geneste [1985 Thèse] 1988; Pelegrin [1986 Thèse] 1995a; 1995b; Perlès 1987; 1991a; 2004; Soressi 2002; Audouze \& Janny 2009). La quantification intervient quand la dimension économique des productions lithiques est prise en compte. Pigeot, par exemple, effectue un décompte des lames issues de chacun des blocs remontés, y compris les lames absentes, les 
outils et les éclats retouchés, afin de comparer la productivité des tailleurs de chaque niveau de compétence ([1983 Thèse] 1987: 72-76).
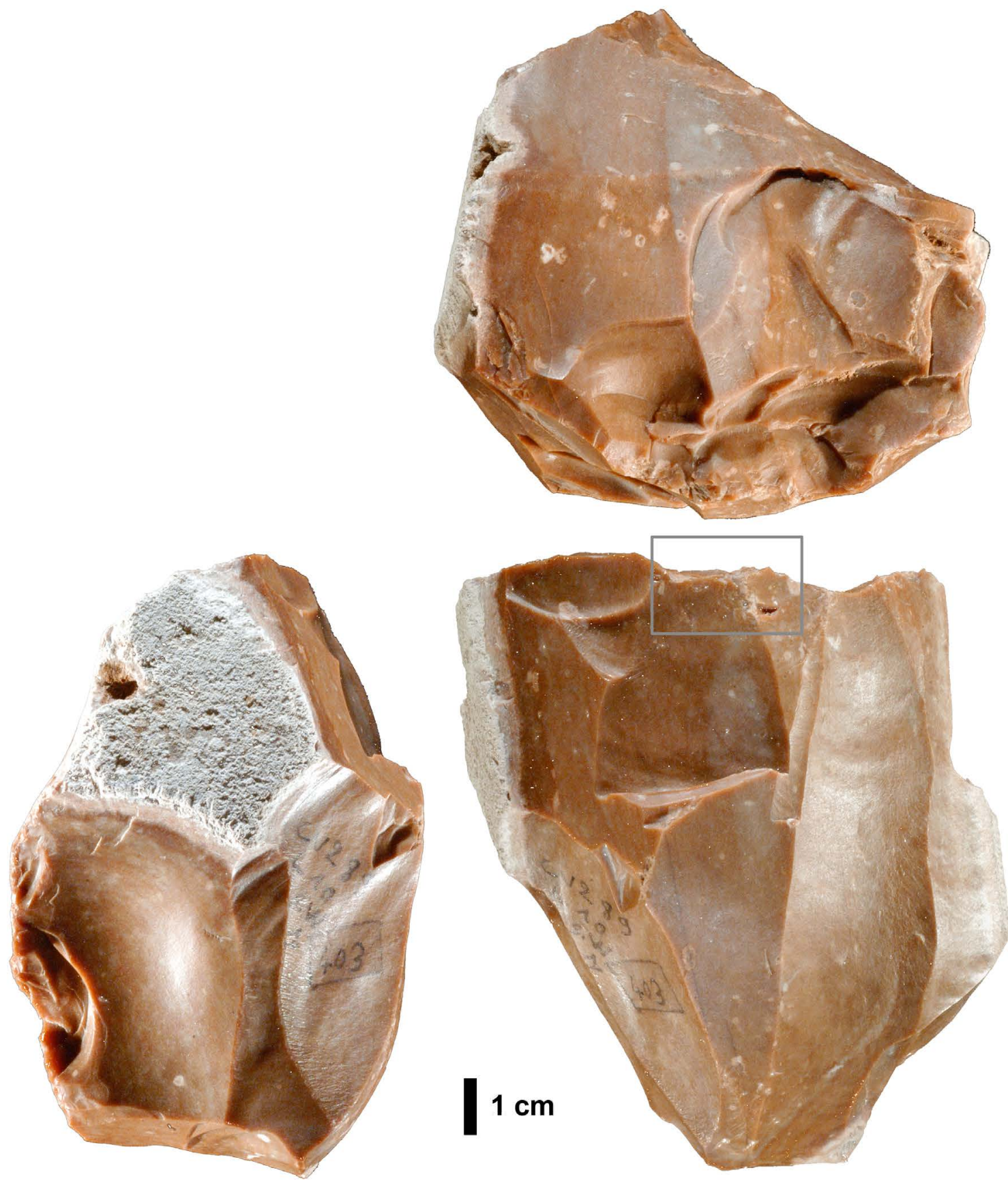

Figure 24. Ce nucléus de Corbiac-Vignoble présente sur la face avant un ou deux enlèvements laminaires témoignant d'un savoir-faire accompli, puis sur le plan de frappe un écrasement de la lèvre et des cercles de percussions dures qui témoignent d'une insistance terminale maladroite attribuable à un apprenti (Bordes \& Bachellerie sous presse, cliché Photo de Jugie, MNP).

Figure 24. That core from Corbiac-Vignoble exhibits on the front face one or two blade removals that attest to an accomplished know-how, but a crushing of the edge and rings, characteristic of hard stone percussion on the striking platform, are traces of an unskilled final insistence attributed to an apprentice (Bordes and Bachellerie in press, Photo by Ph. Jugie, MNP). 
a)
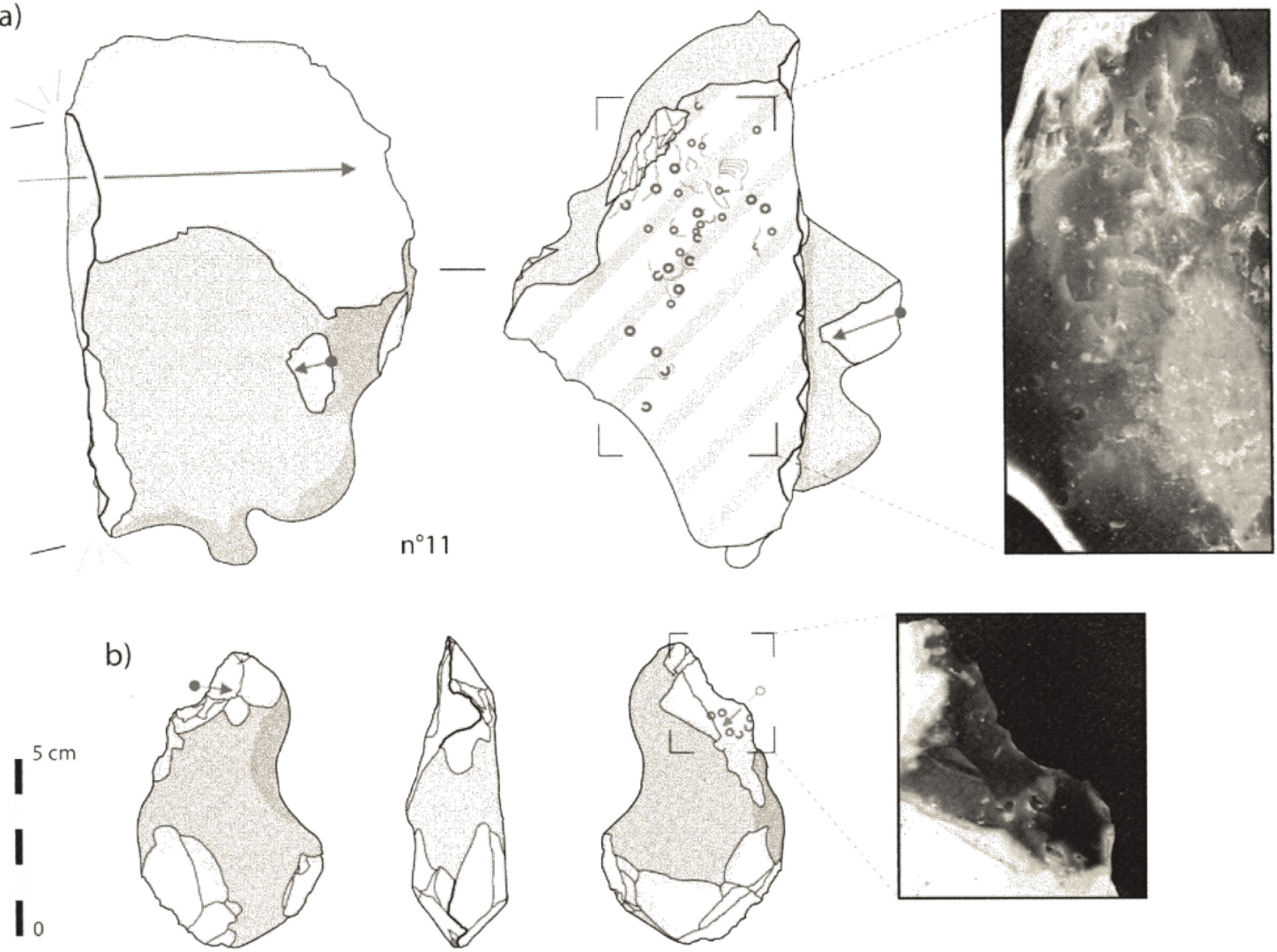

n³9 (unité 3)
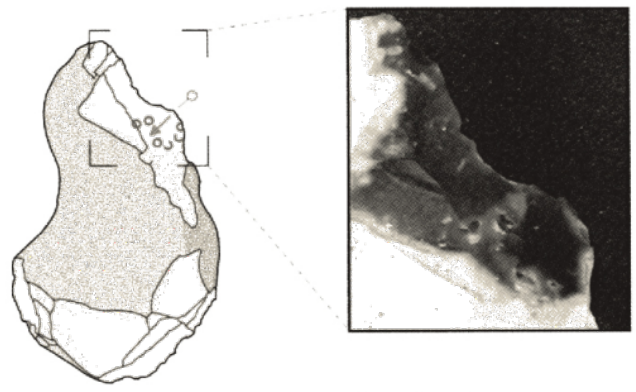

- Cône incipient

c)
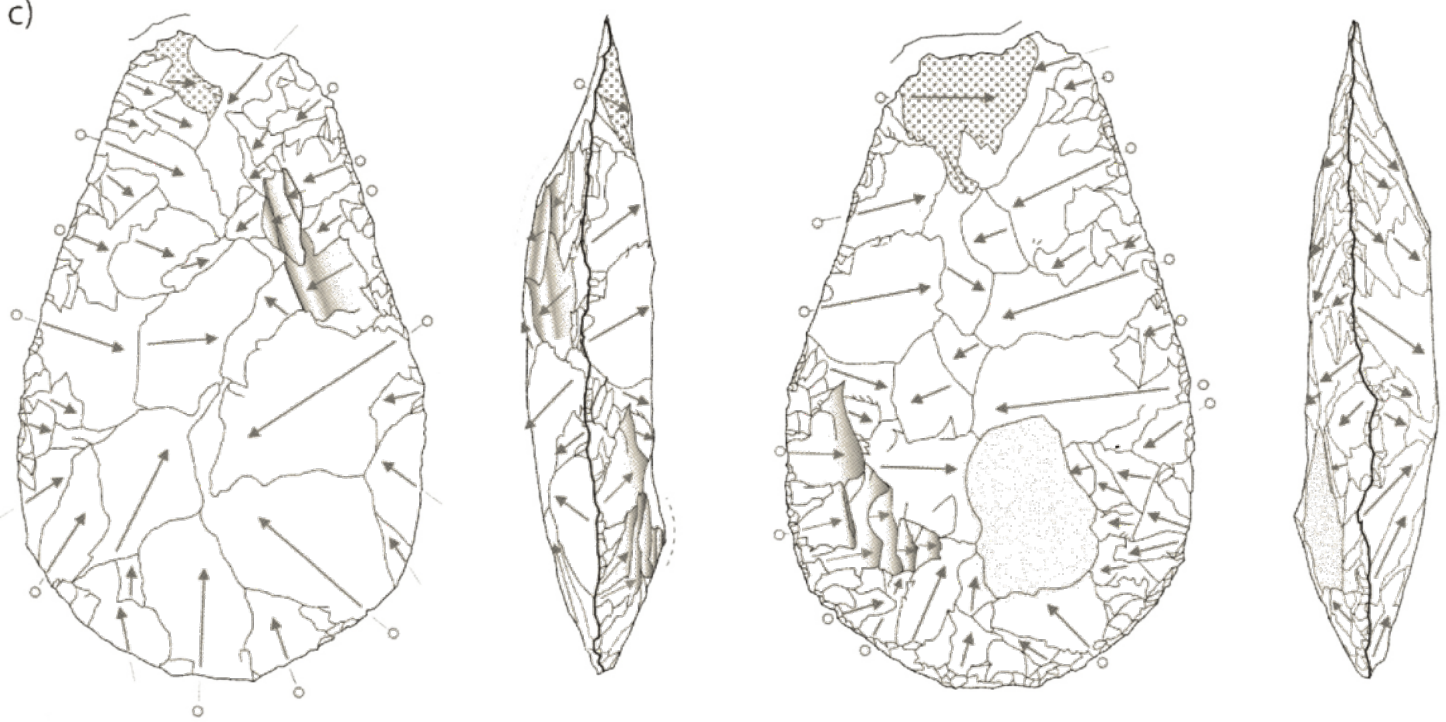

n99 (unité 4u)
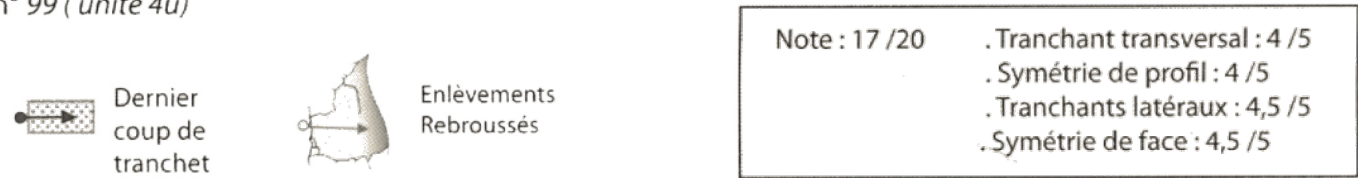

Figure 25. À Boxgrove-EarthamPit (West Sussex, Angleterre) - a) et b) : deux cas évidents de savoir-faire très faible, propres aux tailleurs « malhabiles ", sur blocs peu modifiés. La comparaison avec le biface $n^{\circ} 99$ laisse deviner toute l'étendue d'expression d'un savoir-faire différent (Leroyer 2016: 401, fig. 98).

Figure 25. At Boxgrove-Eartham-Pit (West Sussex, England) - a) and b): two obvious cases of a really poor know-how, characteristic of "unskilled” knappers. The comparison with biface $n^{\circ} 99$ let guess the full gap between a different know-how (Leroyer 2016: 401, fig. 98). 
Les nombreuses expérimentations effectuées depuis plus de 25 ans ont aussi donné lieu à quelques travaux quantitatifs (Lycett \& Chauhan 2010). Aujourd'hui un programme expérimental ayant pour but de compter et mesurer les différents types d'éclats produits durant un débitage Levallois a débuté en 2005 et se poursuit en s'élargissant aux différents modes de production du Paléolithique moyen (Brenet et al. 2009; Roussel et al. 2009). Un des résultats a été de montrer une variabilité marquée, qualitative et quantitative, entre les différents expérimentateurs, mais aussi entre les modalités unipolaire et centripète, dont une plus grande productivité du Levallois centripète (Brenet et al. 2009: 60-64). Des comparaisons avec les assemblages de niveaux archéologiques du Sud-Ouest ont abouti à de premiers résultats sur différents types d'occupation en fonction des modes d'acquisition des matières premières et d'exportation des produits finis (Geneste 1991) (Figure 26). C'est là une description incomplète d'une thématique en développement exponentiel dont nous avons voulu évoquer la richesse.

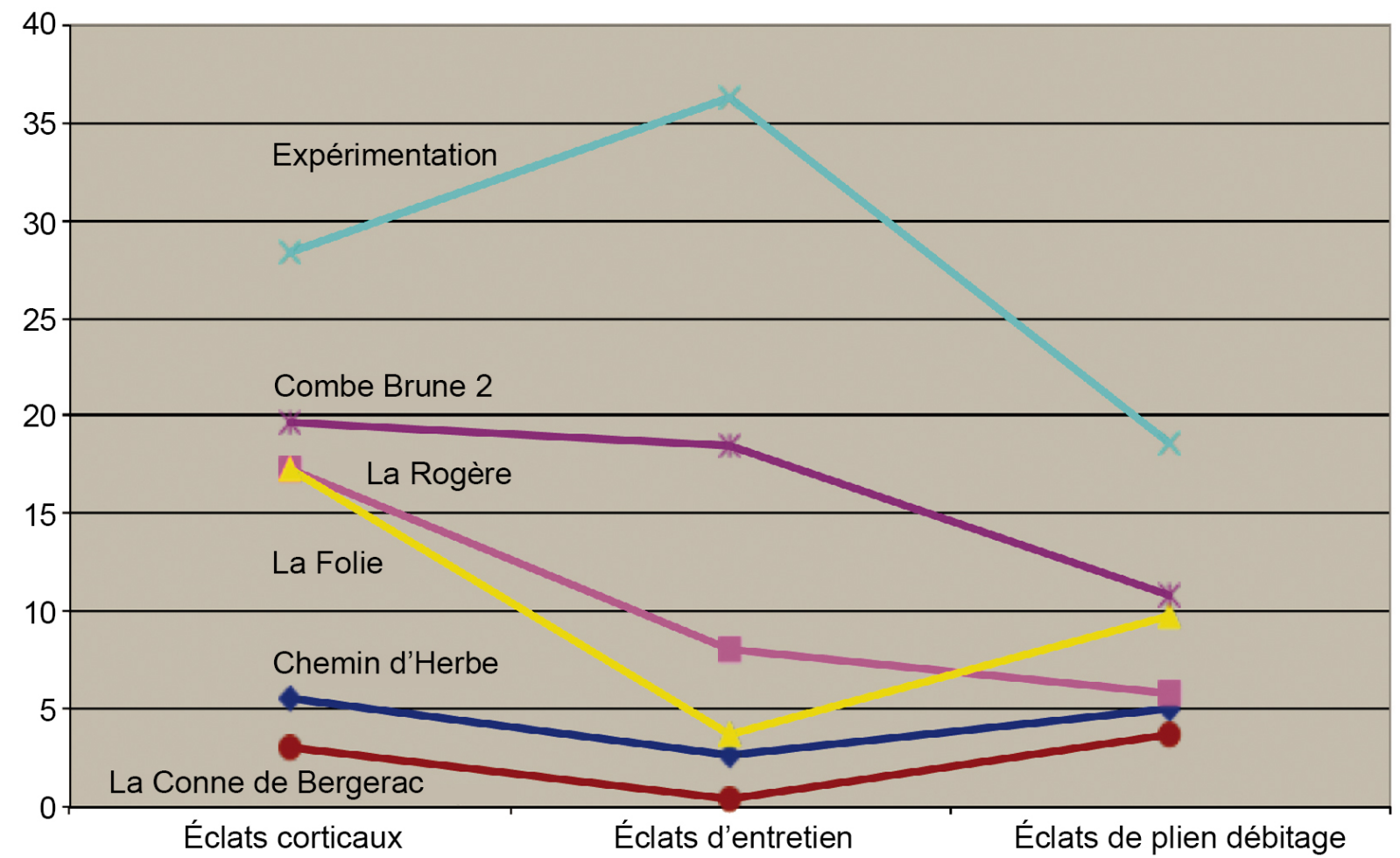

Figure 26. Comparaison en pourcentage de la production expérimentale Levallois centripète avec plusieurs ensembles lithiques de conception levallois. Les productions archéologiques témoignent de comportements économiques variés qui intègrent l'apport de nucléus parfois en partie exploités, la production, la consommation et l'exportation de certains supports produits (d'après Brenet et al. 2009, Les Nouvelles de l'Archéologie: 118, fig. 2).

Figure 26. Comparing in percentage the experimental production of centripetal Levallois flakes and several lithics assemblages of Levallois conception. The archaeological productions attest various economic behaviors that include supply of cores, sometimes partly knapped, production, consumption and export of some of the blanks produced (after Brenet et al. 2009, Les Nouvelles de l’Archéologie: 118, fig. 2).

\section{Avec ou sans la chaîne opératoire : vers une Paléohistoire}

Une époque s'achève et sera close avec la publication des dernières monographies des sites magdaléniens du Bassin parisien, un temps où l'accent a été mis sur les études synchroniques. L'évolution sur le temps long redevient une problématique théorique majeure, promue, entre autres, par deux préhistoriens dans des directions différentes bien que les deux démarches soient placées sous le signe de l'Histoire et abordent le problème de l'interprétation des changements diachroniques. 
B. Valentin (2007; 2008a; Audouze \& Valentin 2010) élabore une paléohistoire qui, à partir des acquis du temps court palethnographique des occupations, vise à mettre en évidence les dynamiques de transformation séquentielles qui mènent d'une tradition technique à une autre, et à en comprendre les mécanismes au sein d'un temps « moyen » d'ampleur décennale ou séculaire (grosso modo la "conjoncture » pour utiliser les termes braudéliens). Cette démarche paléohistorique a été appliquée, par exemple, au processus d’azilianisation pendant le Tardiglaciaire du Bassin parisien, pour lequel les données technologiques étaient suffisamment nombreuses (Valentin 2008a; 2008b). La chaîne opératoire s'y insère comme méthode standard d'analyse des assemblages lithiques, assortie d'une analyse très fine des modifications des armatures de projectile. Elles conduisent à identifier les transformations induites dans le processus général de production dès le débitage des supports (Figure 27). Ces transformations sont mises ensuite en regard des changements reconnus dans l'environnement, la faune, les stratégies et techniques de chasse pour tenter d'expliquer l'apparition de l'azilien dans le Bassin parisien, enrichissant ainsi un modèle proposé naguère par Pelegrin (Pelegrin 2000).

\section{Le cas d'Hangest}

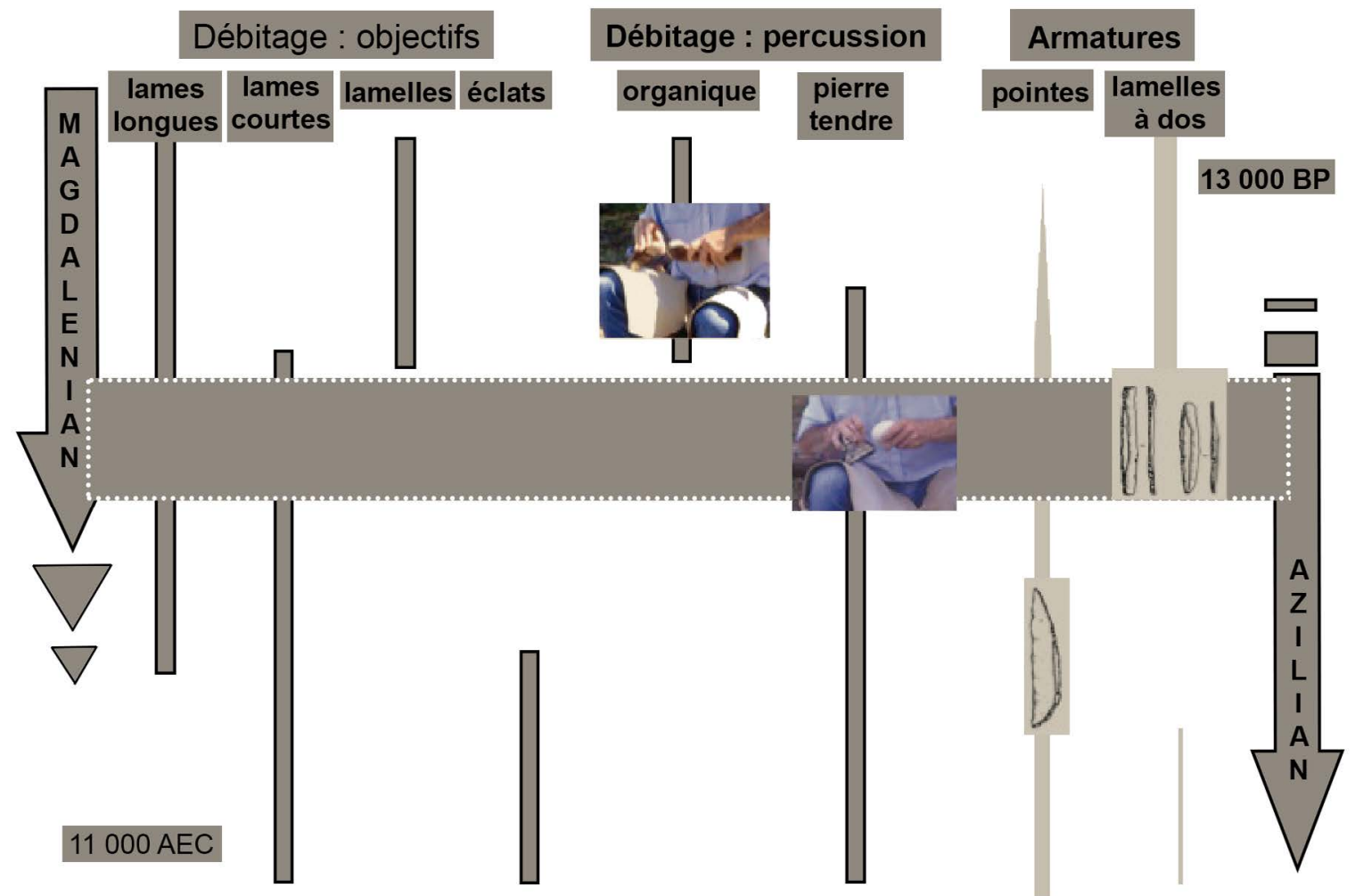

Figure 27. Pour B. Valentin le niveau inférieur d’Hangest III-sur-Somme (France), situé à la transition entre le Magdalénien final et l'Azilien ancien, est un bon exemple d'un processus d'évolution culturelle fait de combinaisons progressives entre anciennes et nouvelles façons de faire. On trouve encore dans ce niveau les lamelles à dos magdaléniennes, mais dans leur grande majorités les lames sont désormais courtes et produites au percuteur de pierre tendre, ce qui sera la règle à l'Azilien; il manque encore les lames à retouche rasante aziliennes et il n'existe qu'une seule bipointe alors qu'elles vont devenir courantes (Valentin 2007: 114 d'après Audouze \& Valentin 2010: 38, fig. 3).

Figure 27. For B. Valentin, the lower level of d'Hangest III-sur-Somme (France), dated from the transition between the late Magdalenian and the earlier Azilian, is a good example of a process of cultural evolution made of progressive combinations between old and new ways of doing. One still finds Magdalenian backed bladelets in this level. But as a whole, blades are short and produced with soft stone hammer, which will be the rule during Azilian. Blades with low angle retouch are still missing and only one bipoint has been found while they will become common (Valentin 2007: 114 d’après Audouze \& Valentin 2010: 38, fig. 3). 
De son côté, la théorie de Boëda renouvelle la conception de tendance tout en se réclamant de Leroi-Gourhan pour l'intégration du geste dans le concept d'outil. Elle appartient à une perspective évolutionniste originale totalement différente des approches anglo-saxonnes (Boëda 2013: 35 et seq.) et repose sur un paradigme venu de la philosophie des techniques et sur les théories de G. Simondon (Simondon [1958] 1989) et Y. Deforge (Deforge 1985; 1996). Elle explique l'évolution des industries en fonction de lignées techniques évolutives qui se succèdent selon des cycles allant de l'objet technique abstrait, dont les parties fonctionnent de façon indépendante, à l'objet concret « dont tous les éléments sont en synergie parfaite et ne peuvent plus être modifiés. Les changements progressifs au sein de chaque lignée peuvent donc être interprétés dans le sens d'une adéquation de plus en plus étroite entre forme et fonction " (Perlès 2016: 227). Boëda s'oriente vers une compréhension structurelle de l'évolution en cherchant ce qui lie les objets en dépit du fait qu'ils changent. Pour lui, «toute évolution dans la lignée est à la fois le reflet du potentiel évolutif du principe structurel de la lignée, et d'une nouvelle réponse à des contraintes inédites. Cette notion de couplage implique nécessairement une coévolution de l’homme et de la technique, cette dernière étant régie par des «lois d’évolution » (Gallay 2013). (Les anglophones trouveront une utile introduction à la Techno-logique dans Frick \& Heckert (2014), notamment un lexique qui fait défaut dans la publication.) La séquence chronostratigraphique du tell d'Umm el Tlel (Syrie) qui s'étend du Néolithique à l'Acheuléen et couvre 800000 ans sans hiatus majeur lui a permis de mettre en application sa théorie et de construire une paléo-histoire technique des modes de productions et de leurs outils de l'Amoudien (300 000 ans BP) à l'Ahmarien et à l'Aurignacien (25 000 ans BP) (Boëda et al. 2001, Boëda 2013).

Cette théorie présente un problème d'interprétation soulevé par Roux (Gallay 2013), notamment la construction d'une paléohistoire à l'échelle du temps de l'évolution, qui conduit Boëda à se servir de lignées théoriques pour retracer des lignées culturelles. On peut toutefois considérer que, dans l'application qu'il en fait sur le site d'Um el Tlel, l'arrivée dans l'oasis puis le départ de groupes humains successifs et le retour de leurs probables descendants, attestés par les différentes traditions techniques lithiques qui se succèdent en alternance, représentent des faits relevant d'une paléohistoire du temps long braudélien (d'ampleur pluriséculaire à millénaire). En dépit de son accès difficile, l'influence de cette œuvre se fait déjà sentir chez les préhistoriens français travaillant sur le Paléolithique ancien et moyen (Forestier 1996; Lourdeau 2012; Lourdeau et al. 2014).

\section{Les secrets d'un succès}

Dans un article d'épistémologie, Pigeot donne une explication de l'étonnant succès du terme chaîne opératoire. Pour elle, c'est « une expression suffisamment floue et suffisamment précise, comme toutes les expressions métaphoriques qui «fonctionnent », pour bénéficier d'une richesse sémantique maximale. Ce concept possède tout le contenu sémantique de l'acte technique (sans être frappé de sa dévalorisation dans l'approche anthropologique) » (Pigeot 2011: 149-171). Son énonciation dans la formule de Leroi-Gourhan contient en germe toute sa richesse (Leroi-Gourhan 1964: 164) : « cinétique du geste, externalité par l’outil et les séries opératoires, l'organisation en termes de processus de la chaîne technique soulignée par le lien organique et métaphorique des maillons qui s'enchaînent et se succèdent (dans le temps et dans l'espace)... syntaxe, c'est-à-dire les règles sociales, les lois de l'action, ...[qui sont du] domaine de la méthode, du savoir, et des connaissances ; fixité et souplesse « qui renvoient à la culture et au style, à l'individu » (Pigeot 2011: 152).

La chaîne opératoire inscrit l'acte technique dans un processus et à ce titre l'inscrit «dans presque tous les plans de l'organisation sociale et de la planification cognitive » 
(Pigeot 2011: 156). Elle réintroduit la personne humaine - un des grands absents de la documentation archéologique - comme acteur médiateur du social. "C’est la même chaîne

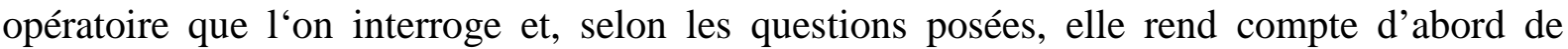
données purement techniques (le comment faire), (...) économiques sur l'objectif (le pourquoi faire) ...qui renvoient à la tradition culturelle, à la fonction utilitaire des activités de fabrication. Mais il suffit de changer (...) de position et de point de vue [pour regarder] non plus les produits et les déchets de l'activité mais la main qui les manipule et agit, l'acteur qui pense, anticipe et décide avant d'agir. Grâce à cette redécouverte de l'humain pensant, la chaîne opératoire actualise le fort potentiel de la technologie » (Pigeot 2011: 157-158).

Flexibilité, vision dynamique des processus et connexion structurelle de la sphère technique à toutes les dimensions humaines font de cet outil analytique une efficace machine à penser les processus de production et leur place dans les sociétés du passé.

\section{Pour conclure}

Au terme de cette longue histoire introductive, rappelons les deux courants qui ont développé cette procédure analytique à la française. Ils sont nés de façon indépendante puis se sont croisés et mutuellement enrichis.

À un niveau plus ou moins conscient, ceux qui ont bénéficié de l'enseignement oral de Leroi-Gourhan, ont le sentiment qu'il existe une science de l'homme qui se déploie selon des problématiques, des modalités d'observation et de modélisation, et selon des échelles diverses en fonction des questions posées. Pour eux il n'y a pas de limites à l'élargissement de la grille d'analyse sinon celles imposées par les données et les traditions disciplinaires.

Tixier, de son côté, a légué à ses élèves une approche sensible de la pierre taillée motivée par la volonté de reconstruire les comportements et mettant l'accent sur les intentions des tailleurs et sur l'intérêt d'une connaissance pratique de la taille.

Au delà de ces deux courants interféconds, de multiples interactions ont conduit à une appropriation réciproque de ces héritages donnant à la chaîne opératoire « à la française » ses caractéristiques :

- l'interaction des ethnologues de la « technologie culturelle » et des préhistoriens sur cette approche dont le développement leur a été en partie commun ;

- un arrière-plan anthropologique implicite mais réellement présent dans la démarche des préhistoriens, devenu plus explicite avec l'introduction des données ethnoarchéologiques ;

- une recherche empirique rigoureuse et détaillée à la base de la procédure,

- adossée à une recherche expérimentale reproductible (caractérisation fine des gestes et des critères permettant d'identifier les techniques, les méthodes de taille, les types de produits, les ratés...);

- la création de concepts originaux pour la production des outils lithiques (économie du débitage, économie de la matière première, "structure volumétrique » synthétisant un mode de production ...);

- la prise en compte des facteurs psychomoteurs dans l'analyse du comportement technique, ce qui a conduit à distinguer les savoir-faire des connaissances, puis à systématiser les degrés de savoir-faire dont l'identification renvoie à des degrés d'apprentissage pour le Paléolithique, à des degrés de spécialisation pour le Néolithique, et parfois même à des individus ;

- des modèles a postériori plutôt que des modèles théoriques a priori ;

- une diversification des applications par extension à des domaines voisins (autres matériaux que la pierre, mais aussi certaines activités économiques ou sociales, périodes 
récentes, et dans un autre ordre d'idées, cognition à partir de l'apprentissage par

exemple).

Dans cet article, nous nous sommes efforcées de retracer un parcours, de montrer les différents stades de développement en France de la chaîne opératoire et de rendre brièvement compte des multiples directions de recherches dans lesquelles elle s'est trouvée partie prenante ou pièce maîtresse. Nous avons esquissé quelques uns de ses plus récents développements. Que tous ceux qui n'y trouvent pas leur nom nous pardonnent - notamment les Néolithiciens. Le nombre des publications a suivi une courbe exponentielle rendant un compte-rendu exhaustif impossible. Il y a de notre part une inévitable subjectivité à nous remémorer cinquante années de recherches dont nous avons été tantôt actrices tantôt spectatrices fascinées par ces moments alternés de lente maturation, d’intenses réflexions collectives et de foisonnement de résultats. (On ne trouvera pas de commentaires ou de réponses aux critiques anglo-saxonnes qui mériteraient à elles seules un long article. Mais on peut se référer à l'excellent article de C. Perlès (2016) «La technologie lithique de part et d'autre de l'Atlantique ».)

\section{Remerciements}

Nos remerciements vont à Catherine Perlès, Jacques Pelegrin, Nathan Schlanger, Nejma Goutas et Philippe Soulier pour leurs utiles commentaires, leurs critiques et leurs suggestions; notre gratitude va aussi à tous ceux qui nous ont aimablement autorisé à reproduire leurs illustrations.

\section{Références}

Alix, C. 2001a, Exploitation du bois par les populations néo-eskimo entre le nord de l'Alaska et le Haut Arctique canadien. Thèse de doctorat, Université de Paris-I - Panthéon Sorbonne, Paris, Vol. 1, texte: 434 p. \& vol. 2, planches \& annexes: 18 p. (in French) ("Wood Use by the Neo-Eskimo Populations between North Alaska and Canadian Frozen Arctic")

Audouze, F., Cahen, D., Keeley, L. \& Schmider, B. 1981, Le site magdalénien du Buisson Campin à Verberie (Oise). Gallia Préhistoire, 24(1): 99-143. (in French) ("The Magdalenian Site of Buisson-Campin at Verberie (Oise)”) doi:10.3406/galip.1981.1667

Audouze, F. \& Cahen, D. 1982, Décryptage d'un habitat magdalénien de plein air. In: Les Habitats du Paléolithique supérieur, colloque international en hommage au Pr. LeroiGourhan, Pré-Actes du Colloque de Villerest 22-24 juin 1982, Roannes-Villerest: p. 210-220. (in French) (Deciphering an Open Air Magdalenian Dwelling)

Audouze, F. \& Cahen, D. 1984, L’occupation Magdalénienne de Verberie et sa chronologie. In: Upper Palaeolithic Settlement Patterns in Europe (Berke, H., Hahn, J., Kind, C.J., Eds.), Archaeologia Venatoria Verlag, Tübingen: p. 143-158. (in French) (The Magdalenian Occupation at Verberie and its Chronology)

Audouze, F., Karlin, C. Cahen, D, Croisset, D. de, Coudret, P, Larrière, M., Masson, P., Mauger, M., Olive, M., Pelegrin, J., Pigeot, N., Schmider, B. \& Taborin, Y. 1988, Taille du silex et finalité du débitage dans le Magdalénien du Bassin Parisien. In: De la Loire à l'Oder, Les civilisations du Paléolithique final dans le nord-ouest européen, Actes du colloque international de Liège 1985 (Otte, M., Ed.), BAR International Series Vol. 444, British Archaeological Reports, Oxford: p. 55-84. (in French) ("Flint Knapping and Finality of « Debitage » in the Paris Basin Magdalenian”) 
Audouze, F. \& Janny, F. 2009, Can we Hope to Identify Children's Activities in Upper Palaeolithic Settlements? In: Engendering Prehistoric 'Stratigraphies' in the Aegean and the Mediterranean. Proceedings of an International Conference University of Crete, Rethymno 2-5 June 2005, Kopaka ed., Aegeum, 30: 167-173. (en anglais) ("Pouvons nous espérer identifier l'activité des enfants dans les campements du Paléolithique supérieur ?")

Audouze, F. 2010, Domesticity and Spatial Organization at Verberie. In: The Magdalenian Household: Unraveling Domesticity (Zubrow, E., Audouze, F. \& Enloe, J.G, Eds.), IEMA Distinguished Monograph Series, SUNY Press, Albany: p. 145-175. (en anglais) ("Vie domestique et organisation spatiale à Verberie")

Audouze, F. \& Valentin, B. 2010, A Paleohistorical Approach to Upper Paleolithic Structural Changes. In: Toward an Eventful Archaeology (Bolender, D. J., Ed.), The Institute for European and Mediterranean Archaeology Distinguished Monograph Series, SUNY Press, Albany, NY: p. 29-47. (en anglais) ("Une approche paléohistorique des changements structurels du Paléolithique supérieur”)

Audouze, F. \& Cattin, M.-I. 2011, Flint Wealth Versus Scarcity. Consequences for Magdalenian Apprenticeships. Lithic Technology, 36(2): 109-126. (en anglais) (“Abondance ou manque de silex. Conséquences pour les apprentissages au Magdalénien”) doi:10.1179/lit.2011.36.2.109

Averbouh, A. 2000, Technologie de la matière osseuse travaillée et implications palethnologiques : l'exemple des chaines d'exploitation du bois de cervidé chez les Magdaléniens des Pyrénées. Thèse de doctorat, Université de Paris I, Paris, 500 p. (in French) ("Technology of Worked Bone Material and Palethnological Implications: the Example of the Working Antlers Chains among Pyrenean Magdalenians”)

Averbouh, A., Coord.. 2010, Multilingual lexicon of bone industries. Retreived 1 September 2017. (en anglais) ("Lexique multilangue de l'industrie osseuse")

URL: http://gdreprehistos.cnrs.fr/accueil/les-productions/lexique-multilingue/

Averbouh, A. 2014, Le travail des matières osseuses et les productions associées. In: Un automne à Pincement, le campement magdalénien du niveau IV20 (Julien, M. \& Karlin, C., Eds.), Société préhistorique Française, Paris: p. 135-170. (in French) (“The bone materials process and associated productions”)

URL: http://www.prehistoire.org/shop_515-31884-0-0/m57-2014-un-automne-apincevent-le-campement-magdalenien-du-niveau-iv20-m.-julien-et-c.-karlin-brcollectif.html

Averbouh, A. Ed. 2016, Multilingual Lexicon of Bone Industry, Version 2 (Français-AnglaisItalien-Espagnol, Allemand, Hongrois, Polonais, Russe, Bulgare, Roumain, Portugais, Danois). Avec la collaboration de N. Akhmetgaleeva, A. Balasescu, A. Boguzewski, A. Choyke, M. Christensen, M. Evora, N. Goutas, G. Ledosseur, Cl. Letourneux, S. Lev, L. Manca, M. Margarit, K. Mazurié, A. Pasquini, N. Skakun, J.-M. Tejero, Z. Toth, M. Zhilin \& P. Zidarov. GDRE Prehistos Archaeological Studies II, Hors série Préhistoire de la Méditerrannée, Editions APPAM, Aix-en-Provence: 131 p. (multilingue) (“Lexique multi langues de l'industrie osseuse, Version 2”)

Averbouh, A., Goutas, N. \& Marquebielle, B. 2016, Rod Debitage by Extraction: An Overview of Different Cases Identified for the Upper Palaeolithic and the Mesolithic in Europe. In: Prehistoric exploitation of hard animal materials, Innovation in the production and use of equipment in hard animal materials: origins and consequences in 
Palaeolithic to Mesolithic societies (Averbouh, A., Tejero, J.-M., Goutas N. \& Christensen, M., Eds.), Quaternary International, 403: 57-67. (en anglais) (“Débitage de baguette par extraction : un aperçu de différents cas identifiés au Paléolithique supérieur et au Mésolithique en Europe”) doi:10.1016/j.quaint.2015.08.072

Bailly, M. 2002, La flèche et l'éclat : production et consommation des outillages lithiques taillés de la fin du Néolithique au début de l'âge du Bronze entre Saône et Rhône (26002000 av. J.-C.). Thèse de doctorat, Université de Franche-Comté, Besançon, 507 p. (in French) ("The Arrow and the Flake: Production and Consummation of Knapped Lithic Tools from the End of Neolithic to the Beginning of Bronze Age, between Saône and Rhône (2600-2000 B.C.)”)

Balfet, H. 1975, Technologie. In: Éléments d'ethnologie. vol. 2. Six Approches (Creswell, R., Ed.), Armand Colin, Paris: p. 44-80. (in French) (“Technology”)

Balfet, H., Ed. 1991, Observer l'action technique. Des chaînes opératoires, pourquoi faire ? Edition du Centre national de la recherche scientifique (CNRS), Paris, 191 p. (in French) (“To Observe Technical Action. Chaînes opératoires to do what?”)

Bar Yosef, O. \& Peer, P. van 2009, The Chaîne Opératoire Approach in Paleolithic Archaeology. Current Anthropology, 50(1): 103-131. (en anglais) (“Approche de la chaîne opératoire en archéologie du Paléolithique”) doi:10.1086/592234

Beyries, S. \& Joulian, F. 1990, L'utilisation d'outils chez les animaux : chaines opératoires et complexité technique. Paléo, 2: 17-26. (in French) ("The use of tools among animals: chaînes opératoires and technical complexity”) doi:10.3406/pal.1990.986

Beyries, S. 2002, Le travail du cuir chez les Tchouktches et les Athapaskans : Implications ethno-archéologiques. In: Le travail du cuir de la préhistoire à nos jours, Actes des XXIIème rencontres internationales d'archéologie et d'histoire d'Antibes (AudouinRouzeau, F. \& Beyries, S, Eds.), Editions Association pour la promotion et la diffusion des connaissances archéologiques (APDCA), Antibes: p. 11-12. (in French) ("The Skin Process among Tchouktches and Athapaskans: Ethno-archaeological Implications”)

Beyries, S., Vasil'ev, S., David, F., Karlin, C., D’iatchenko, V.I. \& Tchesnokov, Y.V. 2002, Tentative of Reconstruction of Prehistoric Skin Processing. Archaeological, Ethnology and Anthropology of Eurasia, 2(10): 79-86. (en anglais) ("Tentative de reconstruction d'un travail des peaux préhistorique”)

Beyries, S. \& Rots, V. 2006, Contribution de l'ethnoarchéologie du travail du cuir aux analyses d'usure macro- et microscopiques. Premières applications au site magdalénien de Verberie. In: Habitats et peuplements tardiglaciaires du Bassin Parisien (Valentin, B., Ed.), Projet Collectif de Recherche Programme P7 et P8, Autorisation plurianuelle 2006-2008, Rapport d'activités pour 2006, UMR 7041, Nanterre: p. 50-65. (in French) ("Contribution of Ethnoarchaeological Studies of Skin Processing to Macro- and Microscopic Wear Traces Analysis. First Applications to the Magdalenian site of Verberie”) URL: http://lara.inist.fr/bitstream/handle/2332/1207/PCRTardi2006.pdf

Beyries, S. 2008, Modélisation du travail du cuir en ethnologie : proposition d'un système ouvert à l'archéologie. Anthropozoologica, 43(1): 9-42. (in French) ("Modelisation of the Hide Working Process in Ethnology: Proposal for a System open to Archaeology")

Beyries, S. \& Rots, V. 2008, The Contribution of Ethnoarchaeological Macro- and Microscopic Wear Traces to the Understanding of Archaeological Hide Working Process. In: Prehistoric Technology, 40 Years Later: Functional Studies and Russian 
Legacy (Longo, L. \& Skakun, N., Eds.), BAR International series Vol. 1783, Hadrian Books, Oxford: p. 21-28. (en anglais) (“Contribution de l'étude des micro et macroscopiques traces d'usure ethnoarchéologiques à la compréhension du travail des peaux archéologique”)

Beyries, S. \& Rots, V. 2010, Methods of Reconstruction of the Processes of Hide Treatment in Prehistory: the First Archaeological Applications. In: Unraveling Domesticity (Zubrow, E., Audouze, F. \& Enloe, J., Eds.), The Institute for European and Mediterranean Archaeology Distinguished Monograph Series, State University of New York Press , Albany: p. 269-282. (en anglais) ("Méthodes de reconstruction des processus de traitement des peaux en préhistoire : premières applications archéologiques”)

Bodu, P., Karlin, C. \& Ploux, S. 1990, Who's who? The Magdalenian Flintknappers of Pincevent, France. In: The big Puzzle. International Symposium on refitting Stone Artefacts. Montrepos 1987 (Cziesla E., Eickhoff S., Arts N. \& Winter D., Eds.), Studies in Modern Archaeology Vol. 1, Holos, Bonn: p. 143-163. (en anglais) (“Qui est Qui ? Les tailleurs de silex de Pincevent, France”)

Bodu, P. 1993, Analyse techno-typologique du matériel lithique de quelques unités du site magdalénien de Pincevent : applications spatiales, économiques et sociales. Thèse de doctorat, Université Paris 1- Panthéon Sorbonne, Paris, 3 vol., 1293 p. (in French) ("Techno-Typologic Analysis of the Lithic Material from Some Units of the Magdalenian Site of Pincevent: Spatial, Economic and Social Applications”) URL: http://tel.archives-ouvertes.fr/tel-00577069

Bodu, P., Karlin, C., Julien, M., Enloe, J. G. \& Hardy, M. 2014, L’ensemble sud. In: Un automne à Pincevent. Le campement magdalénien du niveau IV20 (Julien, M. \& Karlin, C., Eds.), Mémoire Vol. 57, Société préhistorique française, Paris: p. 373-462. (in French) (“The South Zone”) URL: http://www.prehistoire.org/shop_515-31884-00/m57-2014-un-automne-a-pincevent-le-campement-magdalenien-du-niveau-iv20-m.julien-et-c.-karlin-br-collectif.html

Boëda, E., Geneste, J.-M. \& Meignen, L. 1990, Identification des chaînes opératoires lithiques du Paléolithique ancien et moyen. Paléo, 2: 43-80. (in French) ("Identification of Lithic Chaînes Operatoire from the Ancient and Middle Paleolithic”) doi:10.3406/pal.1990.988

Boëda, E. 1992, Approche de la variabilité des systèmes de production lithique des industries du Paléolithique inférieur et moyen : chronique d'une variabilité attendue. Techniques \& Culture, 17-18: 37-79. (in French) (“Approach of the Variability of Lithic Production Systems of Ancient and Middle Paleolithic industries: Chronic of an Expected Variability”) doi:10.4000/tc.685

Boëda, E., Griggo, C. \& Soriano-Noël, S. 2001, Différents modes d’occupation du site d’Umm el Tlel au cours du Paléolithique moyen (El Kowm, Syrie centrale). Paléorient, 27(2): 13-28. (in French) ("Various Occupation Ways of Ummel Tlel Site during the Middle Paleolithic (El Kowm, Central Syria)”) doi:10.3406/paleo.2001.4729

Boëda, E., 2013, Techno-logique \& technologie: une paléo-histoire des objets lithiques tranchants, Prigonrieux, @rchrieuxque \& tcom, Nanterre, 264 p. (in French) (“Technologic and Technology: a Paleo-History of Cutting Lithic Tools”) 
Bonte, P. 1986, Les recherches ethnologiques sur les techniques en France après LeroiGourhan. La Pensée, 254: 37-47 (in French) ("Ethnological Researches on Technics in France after Leroi-Gourhan”)

Bordes, J.-G., \& Bachellerie, F. sous presse, Niveaux de savoir-faire et structuration des groupes humains au Paléolithique supérieur ancien : l'exemple de Canaule II (Châtelperronien) et Corbiac-Vignoble II (Aurignacien ancien), Dordogne, France. In: L'apprenti préhistorique : appréhender l'apprentissage, les savoir-faire et l'expertise à travers les productions techniques des sociétés préhistoriques (Klaric L., Ed.). Dolni Vestonice Studies, Brno: p. 52-76 (in French) ("Differential skill-levels and the structure of Early Upper Palaeolithic groups: the example of Canaule II (Châtelperronian) and Corbiac-Vignoble II (Early Aurignacian)”)

Brenet, M., Bourguignon, L., Folgado, M.\& Ortega, I. 2009, Élaboration d’un protocole d'expérimentation lithique pour la compréhension des comportements techniques et techno-économiques au Paléolithique moyen. Les Nouvelles de l'Archéologie, 118: 6064. (in French) ("Elaboration of a Protocol for Lithic Experimentation to Understand Technical and Techno-Economic Behaviours during Middle Paleolithic”) doi:10.4000/nda.941

Bril, B. \& Roux, V. 1993, Compétences impliquées dans l'action. Le cas de la taille des perles en pierre dure (Khambat, Inde). Les objets dans l'action, Raison pratiques, 4: 267-286. (in French) ("Competences Involved in Action. The Case of Hard Stone Pearls Knapping (Khambat, Inde)”)

Bril, B., Smaers, J., Steele, J., Rein, R., Nonaka, T., Dietrich, G., Biryukova, E., Hirata, S. \& Roux, V. 2012, Functional Mastery of Percussive Technology in Nut-cracking and Stone-flaking Actions: Experimental Comparison and Implications for the Evolution of the Human Brain. Philosophical Transactions of the Royal Society: Biological Sciences, 367(1585): 59-74. (en anglais) ("Maitrise fonctionnelle de la technologie de la percussion dans le cassage des noix et le débitage de la pierre : comparaison expérimentale et implications pour l'évolution du cerveau humain”) doi:10.1098/rstb.2011.0147

Cahen, D. \& Karlin, C. 1980, Nouvelles voies pour l'étude des pierres taillées. In: Prehistoire et technologie lithique, 1, table ronde du 11-13 juin 1979, URA (Unité de Recherche Associée) 28, Centre national de la recherche scientifique (CNRS), Valbonne: p. 24-27. (in French) ("New Ways for the Study of Knapped Stones")

Cahen, D. \& Karlin, C. 1980, Les artisans de la Préhistoire. La Recherche, 116: 1258-1268. (in French) ("The CraftsMen of Prehistory”)

Cahen, D., Karlin, C., Keeley, L.H. \& Noten, F. van 1980, Méthodes d'analyse technique, spatiale et fonctionnelle d'ensembles lithiques. Helinium, 20(3): 209-259. (in French) ("Methods for Technical, Spatial and Functional Analysis of Lithic Assemblages")

Cahen, D. et Collectif URA 28. 1982, Tailler ! pour quoi faire ? : Préhistoire et technologie lithique II - Recent progress in microwear studies. Studia Praehistorica Belgica, Vol. 2, Musée royal de l'Afrique centrale, Tervuren, 327 p. (in French) "Knapping ! For what ?: Prehistory and Lithic Technology II".

Cattin, M.-I. 2012, Le site magdalénien de Monruz. 4. La vie quotidienne à travers le travail du silex. Archéologie neuchâteloise Vol. 51, Office du Patrimoine et de l’Archéologie, Neuchâtel: 315 p. (in French) ("The Magdalenian Site of Montruz. 4. The Everyday Life throw the Flint Knapping”) 
Chamoux, M.-N. 1978, La transmission des savoir-faire : un objet pour l'ethnologie des techniques ? Techniques \& Culture, Bulletin de l'Équipe de recherche 191(3): 46-83. (in French) (“Transmission of Know-How: an Object for the Technics Ethnology?") Accédé le 24 juin 2016. doi:10.4000/tc.4995

Chauchat, C. \& Pelegrin, J., 1994, Le premier peuplement de la côte désertique du Pérou. Bulletin de la Société préhistorique française, 91(4): 275-280. (in French) (“The first population of the Peru desertic coast”) doi:10.3406/bspf.1994.9736

Christensen, M. 2015, L'exploitation des matières dures animales chez les chasseurscueilleurs : le cas des nomades marins de Patagonie et de Terre de Feu. Habilitation à diriger des recherches, Université de Paris 1, Panthéon-Sorbonne, Paris, 2 vol., 341 p. (in French) ("The Exploitation of Hard Animal Material among Hunter-gatherers: the Case of the Nomadic Sealers of Patagonia and Tierra del Fuego")

Collectif 1991a, 25 ans d'études technologiques en préhistoire. Actes des $\mathrm{XI}^{\mathrm{e}}$ Rencontres d'Archéologie et d'Histoire d'Antibes, 18-20 octobre 1990, Editions Association pour la promotion et la diffusion des connaissances archéologiques (APDCA), Antibes, 397 p. (in French) ("25 Years of Technological Studies in Prehistory”)

Creswell, R. 1976, Avant-Propos. Techniques \& Culture, Bulletin de l'Équipe de recherche 191(1): 5-6. (in French) ("Foreword")

Creswell, R. 1983, Remarques préliminaires. Techniques \& Culture, 1: 9-10. (in French) (“Preliminary Remarks”) URL: http://journals.openedition.org/tc/1033

Cziesla, E. 1987, L’analyse des raccords ou le concept du dynamisme en préhistoire. Bulletin de la Société préhistorique luxembourgeoise, 9: 77-111. (in French) ("The Analysis of Refits or the Concept of Dynamism in Prehistory")

Cziesla, E., Eickhoff, S., Arts, N., \& Winter, D. (Eds.) 1990, The Big Puzzle: International Symposium on Refitting Stone Artefacts. Studies in Modern Archaeology Vol. 1, Holos, Bonn, 684 p. (en anglais) ("Le grand puzzle : Symposium international sur le remontage d'artefacts lithiques”)

Dauvois, M. 1976, Précis de dessin dynamique et structural des industries lithiques préhistoriques. Editions P. Fanlac, Perigueux, 262 p. (in French) (“Abstract of dynamic and structural drawing for lithic industries”)

David, E. 2004a, Technologie osseuse des derniers chasseurs préhistoriques en Europe du Nord (Xe-VIIIe millénaires avant J.-C.) : Le Maglemosien et les technocomplexes du Mésolithique. Thèse de doctorat, Université de Nanterre-Paris X, Nanterre, 666 p. (in French) ("Bones Technology of last Prehistoric Hunters in North Europe $\left(10^{\text {th }}-8^{\text {th }}\right.$ millennium B.C.): The Maglemosian and the Mesolithic Technocomplexes”)

David, E., 2004b, Fiche : Transformation des matières dures d'origine animale dans le Mésolithique de l'Europe du Nord. In: Fiches de la Commission de Nomenclature de l'Industrie Osseuse, Cahier XI Matières et Techniques (Ramseyer, D., Ed.). Industrie de l'Os Préhistorique, Editions de la Société Préhistorique Française, Paris: p. 113-149. (in French) ("Card: Transformation of Hard Animal Material during the Mesolithic of Northern Europe”)

Delbos, G. \& Jorion, P. 1987, La transmission des savoirs. Editions de la Maison des Sciences de l'Homme, coll. Ethnologie de la France, Paris, 310 p. (in French) (“The Transmitting of Knowledges”) 
Delneuf, M. 1991, Un champ particulier de l'expérimentation en céramique : les ateliers de poterie traditionnelle du Nord-Cameroun. In: Vingt-cinq ans d'études technologiques en préhistoire:bilan et perspectives, Editions Association pour la promotion et la diffusion des connaissances archéologiques (APDCA), Juan-les-Pins: p. 65-82. (in French) (“A Special Wide Field of Experimentation in Ceramic: Traditional Poteries Workshops in the North-Cameroon")

Desrosiers, P.M. Ed. 2012, The Emergence of Pressure Blade Making. From origin to modern experimentation, MA, Springer US, Boston, 521 p. (en anglais) (“L’émergence de la production de lames par pression. De l'origine à l'expérimentation moderne”) doi:10.1007/978-1-4614-2003-3

Desrosier, S. 1991, Sur le concept de chaîne opératoire. In: Observer l'action technique:des chaînes opératoires, pourquoi faire ? (Balfet, H., Ed.), Centre national de la recherche scientifique (CNRS), Paris: p. 21-26. (in French) ("About the Concept of Chaîne Opératoire”)

Deforge, Y. 1985, Technologie et génétique de l'objet industriel. Maloine, Paris, 196 p. (in French) (“Technology and Genetic of the Industrial Object”)

Deforge, Y. 1996, La transmission et la préservation des savoir-faire et les enseignements techniques. In: Savoir faire et pouvoir transmettre: transmission et apprentissage (Chevalier, D., Ed.), Maison des Sciences de l'Homme, Paris: p. 197-216. (in French) ("Transmission and Preservation of Knowledges and the Technical Teaching”) doi:10.4000/books.editionsmsh.3853

Djindjian, F. 1990, Nouvelles méthodes pour l'analyse spatiale des sites archéologiques. Histoire \& Mesure, 5(1): 11-34. (in French) ("New Methods for Spatial Analysis of Archeological Sites”) doi:10.3406/hism.1990.1363

Djindjian, F. 2013, Us et abus du concept de « chaîne opératoire » en archéologie. In: L’Age du Fer en Europe. Mélanges offerts à Olivier Buchsenchütz, (Krausz, S., Colin, A., Gruel, K., Ralston, I., Dechezlepetre, T., Eds.), Mémoire Vol. 32, Editions Ausonius, Bordeaux: p. 93-107. (in French) ("Uses and Abuses of the Concept of « Chaîne opératoire » in Archeology”)

Eigeland, L. \& Sternke, F., Eds. 2011, Scarcity’s Apprentice Raw Material Availability and Skill Trasmission in Prehistoric Lithic Technology. EAA symposium, 26-30/03/2008, Vancouver, Lithic Technology, 36(2): 95-221. (en anglais) (“Accès à la matière première pour les apprentis en situation de rareté des ressources et transmission des compétences en technologie lithique préhistorique”) doi:10.1179/lit.2011.36.2.95

Fischer, A. 1989, A Late Palaeolithic "School” of flint-knapping at Trollesgave, Acta Archaeologica, 60: 33-49. (en anglais) ("Une « école » de taille du silex au Paléolithique Supérieur à Trollesgave”)

Fischer, A. 1990, On Being a Pupil of a Flintknapper of 11,000 Years Ago. A preliminary Analysis of Settlement Organization and Flint Technology based on Conjoined Flint Artefacts from the Trollesgave site. In: The Big Puzzle: International Symposium on Refitting Stone Artefacts, Monrepos, 1987 (Cziesla, E., Eickhoff, S., Arts, N. \& Winter, D., Eds.), Holos, Bonn: p. 447-464. (en anglais) (“Être l'élève d’un tailleur il y a 11,000 ans. Une analyse préliminaire de l'organisation du campement et de la technologie lithique fondé sur l'ensemble d'artefacts en silex du site de Trollesgave”) 
Fluzin, P. 2002, La chaîne opératoire en sidérurgie : matériaux archéologiques et procédés. Apports des études métallographiques. In: Aux origines de la métallurgie du fer en Afrique. Une ancienneté méconnue. Afrique de l'Ouest et Afrique centrale (Bocoum, H., Ed.), Mémoire des peuples, UNESCO, Paris: p. 59-91. (in French) ("The Chaîne Opératoire for Siderurgy: Archeological Materials and Process. Metallographic Studies Contribution")

Fluzin, P., Bauvais, S., Berranger, M., Pages, G. \& Dillmann, P. 2011, The Multidisciplinary Approach (Archaeology and Archaeometry) to Bloomsmithing Activities in France: Example Results from the Last Twenty Years. In: The Archaeometallurgy of Iron. Recent Developments in Archaeology and Scientific Research. Dedicated to Professor Radomir Pleiner (Hosek, J., Henry, C. \& Mihok, L., Eds.), The institute of Archaeology of the Academy of Sciences of the Czech Republic (ASCR), Prague: p. 223-236, 311312. (en anglais) ("Etudes multidisciplinaires (archéologique et archéométrique) des activités de fonte en bas fourneaux en France: l'exemple de ces vingt dernières années”) URL: https://halshs.archives-ouvertes.fr/halshs-00670718

Forestier, H. 1996, L’industrie lithique des premiers Mélanésiens de Nouvelle-Calédonie. Journal de la Société des océanistes, 102(1): 71-89. (in French) ("Lithic Industry of the First Melanesians of New-Caledonia”) doi:10.3406/jso.1996.1978

Frick, J.A. \& Herckert, K. 2014, Lithic Technology and Logic of Technicity, Mitteilungen des Gesellshaft für Urgeschichte, 23, Universitat Tubingen, Tubingen: p. 129-172. (en anglais) ("Technologie lithique et logique de la technicité”)

Gallay, A. 2013, Notes de Lectures, BOËDA, E. Techno-logique et technologie : une paléohistoire des objets tranchants. Archéoédition.com, archeo-gallay. (in French) ("Reading-Notes, BOËDA E., Techno-logic and Technology: a Paleo-history of Cutting Tools”) URL: http://www.archeo-gallay.ch/old/7a_Lectures17.html

Geneste, J.-M. [1985 Thèse] 1988, Analyse lithique d'industries moustériennes du Périgord : une approche technologique du comportement des groupes humains au Paléolithique moyen. Thèse, Université de Bordeaux I, Bordeaux, 682 p. (in French) ("Lithic Analysis of the Mousterian Industries of Perigord: a Technological Approach of the Behaviour of Human Groups during Middle Paleolithic”)

Geneste, J.-M. 1990, Développement des systèmes de production lithique au cours du Paléolithique moyen en Aquitaine septentrionale. In: Paléolithique moyen récent et Paléolithique supérieur ancien en Europe (Farizy, C., Ed.), Association pour la promotion de la recherche archéologique en Ile-de-France (APrAiF), Nemours: p. 203213. (in French) ("Evolution of Lithic Production Systems during the Middle Paleolithic in Northern Aquitaine”)

Geneste, J.-M. 1991, L'approvisionnement en matières premières dans les systèmes de production lithique. La dimension spatiale de la technologie. Treballs d'Arqueologia, 1: 1-36. (in French) ("Supplying of raw material in the lithic production systems. The spatial dimension of technology")

Geneste, J.-M. 2010, Systèmes techniques de production lithique. Variations technoéconomiques dans les processus de réalisation des outillages paléolithiques. Techniques \& Culture, 54-55: 419-449. (in French) ("Technical Systems of Lithic Production. Techno-Economic Variations in the Production Process of Lithic Equipments”) doi:10.4000/tc.685 
Ghesquiere, E. Marcigny, C., Desloges, J. \& Le Gaillard, L. 1997, Le silex à l’Age du Bronze. L'industrie lithique Age du Bronze du site de l'île Tatihou à Saint-Vaast-laHougue (Manche). Revue archéologique de l'ouest, 14(1): 27-47. (in French) ("Flint during the Bronze Age. Lithic Industry Bronze Age of the Tatihou island Site at SaintVaast-La-Hague, Manche”) doi:10.3406/rao.1997.1054

Goutas, N \& Tejero, J.M. 2016, Osseous Technology as a Reflection of Chronological, Economic and Sociological Aspects of Palaeolithic Hunter-Gatherers. A few Examples from key Aurignacian and Gravettian Sites in South-West Europe. In: Prehistoric exploitation of hard animal materials, Innovation in the production and use of equipment in hard animal materials: origins and consequences in Palaeolithic to Mesolithic societies (Averbouh A, Tejero J.M., Goutas N. \& Christensen M., Eds.), Quaternary International, 403: 79-89. (en anglais) ("La technologie osseuse comme reflet des aspects chronologiques, économiques et sociaux de la vie des chasseurscueilleurs du Paléolithique. Quelques exemples de sites clé de l’Aurignacien et du Gravetien dans l'Europe du Sud-Ouest”) doi:10.1016/j.quaint.2015.11.143

Haudricourt, A.-G. 1987, La technologie science humaine. Recherches d'histoire et d'ethnologie des techniques. Éditions de la Maison des Sciences de I'Homme, Paris, 343 p. (in French) ("Technology as Human Science. Research of Technics History and Ethnology")

Hermann, A. 2013, Les industries lithiques pré-européennes deiPolynésie centrale. Savoirfaire et dynamiques techno-économiques. Thèse de doctorat. Université de la Polynésie française, Tahiti, 420 p. (in French) ("Pre-European Lithic Industries of Central Polynesia. Know-how and Techno-Economic Dynamics”)

Hesse, A. 1971, Les Tarterets II, site paléolithique de plein air à Corbeil-Essonnes (Essonne). II Comparaison par le calcul des distributions horizontales des vestiges lithiques. Gallia Préhistoire, 14: 41-46. (in French) ("Tarterets II, Open-air Paleolithic Site at CorbeilEssonnes (Essonne). II Comparison by the Calculation of Horizontal Distribution of Lithic Remains”)

Hill, J.N. \& Gunn, J. 1977, Introducing the Individual in Prehistory. In: The individual in Prehistory: studies of Variability in Style in Prehistoric Technologies (Hill, J.N. \& Gunn, J., Eds.), Academic Press, New York: p. 1-12. (en anglais) (“Apparition de l’individu en préhistoire”)

Hill, J.N. 1978, Individuals and their Artifacts: an Experimental Study in Archaeology. American Antiquity, 43(2): 245-257. (en anglais) ("Les individus et leurs artefacts : une étude expérimentale en archéologie”) doi:10.2307/279248

Holl, A. 1985, Analyse archéologique de la céramique : un cas de la région du Dhar Tichitt (Mauritanie). Techniques \& Culture, 5: 93-110. (in French) ("Archeological Analysis of Ceramic: a Case from Dhar Tichitt area (Mauritania)”) doi:10.4000/tc.957

Horard-Herbin, M.-P. \& Vigne, J.-D., Eds. 2005, Animaux, environnements et sociétés. Collection Archéologiques, Editions Errance, Paris, 192 p. (in French) (“Animals, Environment and Societies”)

Inizan, M.-L. 1976, Nouvelle étude d'industries lithiques du capsien (Collection Raymond Vaufrey, Institut de paléontologie humaine, Paris). Thèse de 3ème cycle EthnologiePréhistoire, Paris X, Nanterre \& Paris, 328 p. (in French) ("New Study of Capsian Lithic Industries (Raymond Vaufrey Collection, Human Paleontology Institut, Paris)”) 
Inizan, M.-L. 1980, Séries anciennes et économie du débitage. In: Préhistoire et Technologie lithique (Tixier, J., Ed.), Centre national de la recherche scientifique (CNRS), Paris: p. 28-30. (in French) (“Old Series and Debitage Economy”)

Inizan, M.-L. 2012, Pressure Debitage in the Old World: Forerunners, Researchers, Geopolitics? Handing on the Baton. In: The Emergence of Pressure Blade Making (Desrosiers, P. M., Ed.), Springer US, Boston: p. 11-42. (en anglais) ("Débitage par pression dans l'Ancien Monde : précurseurs, chercheurs, Géopolitique ? Passer le flambeau”) doi:10.1007/978-1-4614-2003-3_2

Inizan, M.-L., Roche, H. \& Tixier, J. 1992, Technology and Terminology of Knapped Stone, Vol. 3, Cercle de Recherches et d'Etudes Préhistoriques (CREP), Meudon, 127 p. (en anglais) ("Technologie et terminologie de la pierre taillée")

Inizan, M.-L., Reduron, M., Roche, H. \&Tixier, J. 1995, Technologie de la pierre taillée. t. 4, Cercle de Recherches et d'Etudes Préhistoriques (CREP), Paris: 199 p. (in French) (“Technology of Knapped Stone”)

Inizan M.-L., Ballinger, M., Roche, H. Tixier, J. [1995]1999, Préhistoire de la pierre taillée. T. 4 : Technologie de la Pierre taillée, CREP, Meudon, 199 p. (in French) ("Prehistory of Knapped Stone. T.4: Technology of Knapped Stone”)

Johnson, I. 1976, Contribution méthodologique à l'étude de la répartition des vestiges dans des niveaux archéologiques. Mémoire de DES (Diplôme d'Etudes Supérieures), Université de Bordeaux I, Bordeaux, 169 p. (in French) ("Methodological Contribution to Remains Distribution Study in the Archeological Levels")

Johnson, I., 1984, Cell Frequency Recording and Analysis of Artefact Distributions. In: Intrasite spatial Analysis in Archaeology (Hietala, H., Ed.), Cambridge University Press, Cambridge: p. 75-96. (en anglais) ("Enregistrement de la fréquence des groupements et analyse de la distribution des artefacts")

Julien, M. \& Karlin, C. 2007, Variations saisonnières chez des Magdaléniens et des Sibériens : approche ethnoarchéologique. In: Les civilisations du renne d'hier et d'aujourd'hui:approches ethnohistoriques, archéologiques et anthropologiques (Beyries, S. \& Vaté, V., Eds.), éditions Association pour la promotion et la diffusion des connaissances archéologiques (APDCA), Antibes: 163-184. (in French) ("Seasonal Variations among Magdalenians and Siberians: Ethnoarcheological Approach”)

Julien, M., \& Karlin, C., Eds. 2014, Un automne à Pincevent. Le campement du niveau IV20. Mémoire 57 Société préhistorique française. Paris, 639 p. (in French) (“An Autumn at Pincevent. The Settlement of Level IV20”) URL: http://www.prehistoire.org/shop_51531884-0-0/m57-2014-un-automne-a-pincevent-le-campement-magdalenien-du-niveauiv20-m.-julien-et-c.-karlin-br-collectif.html

Julien, M. \& Karlin C. 2014, Un campement résidentiel pour la chasse aux rennes. In: Un automne à Pincevent. Le campement du niveau IV20 (Julien M. \& Karlin, C., Eds.), Mémoire 57, Société préhistorique française, Paris: 587-589. (in French) (“A Residencial Settlement for Hunting Reindeers”)

URL: http://www.prehistoire.org/shop_515-31884-0-0/m57-2014-un-automne-apincevent-le-campement-magdalenien-du-niveau-iv20-m.-julien-et-c.-karlin-brcollectif.html

Julien, M., Karlin, C., Enloe, J.G. \& Hardy, M. 2014, Le nord du campement. In: Un automne à Pincevent. Le campement magdalénien du niveau IV20 (Julien M. \& Karlin, C., Eds.), 
Mémoire 57. Société préhistorique française, Paris: 462-503. (in French) ("The North of the Settlement”) URL: http://www.prehistoire.org/shop_515-31884-0-0/m57-2014-unautomne-a-pincevent-le-campement-magdalenien-du-niveau-iv20-m.-julien-et-c.-karlinbr-collectif.html

Karlin, C. 1966, Le débitage. In: L’habitation magdalénienne $N^{\circ} 1$ de Pincevent près Montereau (Seine-et-Marne) (Leroi-Gourhan, A. \& Brézillon, M.), Gallia Préhistoire, 9(2): 276-279. (in French) (“The Debitage”) doi:10.3406/galip.1966.1264

Karlin, C. 1972, Le débitage. In: Fouilles de Pincevent:essai d'analyse ethnographique d'un habitat magdalénien (la section 36) (Leroi-Gourhan, A. \& Brézillon, M.). Paris, éditions du Centre national de la recherche scientifique (CNRS), supplément à Gallia préhistoire, 7, 2 vol.: 263-277. (in French) (“The Debitage”)

Karlin, C. \& Pigeot, N. 1989, Chasseurs-cueilleurs magdaléniens : techniques de taille et apprentissage de la taille du silex. Le Courrier du CNRS, 73: 10-11. (in French) ("Magdalenian Hunters-Gatherers: Knapping Technics and Flint Knapping Learning”)

Karlin, C., Bodu, P. \& Pelegrin, J. 1991, Processus techniques et chaînes opératoires. Comment les préhistoriens s’approprient un concept élaboré par les ethnologues. In: Observer l'action technique. Des chaînes opératoires, pourquoi faire ? (Balfet, H., Ed.), Editions du Centre national de la recherche scientifique (CNRS): 101-117. (in French) ("Technic Process and Chaînes Operatoires. How Prehistorians Appropriate a Concept Builded up by Ethnologists”)

Karlin, C. 1992, Analyse d'un processus technique : le débitage laminaire des Magdaléniens de Pincevent (Seine-et-Marne). In: Technologia y cadenas opérativas liticas. Réunion internationale 15/18 janvier 1991 (Mora, R., Terradas, X., Paerpal, A. \& Plana, C., Eds.), Tribal d'Arqueologia 1: 125-161. (in French) (“Analysis of a Technical Process: the Magdalenian Blade Debitage at Pincevent (Seine-et-Marne)”)

Karlin, C. Ed. 1992, Le geste retrouvé. Techniques \& Culture, n 17-18 janvier-décembre 1991, éditions EHESS. (in French) (“The Rediscovered Gesture”)

URL: https://journals.openedition.org/tc/680

Karlin C., Pigeot, N. \& Ploux, S. 1992, L'ethnologie préhistorique. La Recherche, 247: 11061115. (in French) ("Prehistoric Ethnology")

Karlin, C. \& Julien, M. 1994, Prehistoric technology: a cognitive science? In: The ancient mind. Elements of Cognitive Archaeology (Renfrew, C. \& Zubrow E.B.W., Eds.), Cambridge University Press, Cambridge: p. 152-164. (en anglais) (“Technologie préhistorique : une science cognitive ?”) doi:10.1017/CBO9780511598388.016

Kintigh, K.W. \& Ammerman, A.J.T. 1982, Heuristic Approaches to Spatial Analysis in Archaeology. American Antiquity, 47(1): 31-63. (en anglais) (“Approches heuristiques de l'analyse spatiale en archéologie”) doi:10.2307/280052

Klaric, L., (Ed.), sous presse, L'apprenti préhistorique : appréhender l'apprentissage, les savoir-faire et l'expertise à travers les productions techniques des sociétés préhistoriques. Dolni Vestonice Studies, Brno, 350 p. (in French) ("The Prehistoric Apprentice: Investigating apprenticeship, know-how and expertise in prehistoric technologies”)

Langlais M. 2013, Productions et équipements domestiques en silex du Magdalénien récent de Verberie - Le Buisson Campin (Oise, France), niveau II-1. Des traditions technoéconomiques aux implications sociales. Rapport non publié. (in French) ("Flint 
Productions and Domestic Equipments of Recent Magdalenian at Verberie -Le Buisson Campin (Oise, France), Level II-I. From the Techno-economic Traditions to Social Implications")

Langlais, M. sous presse, De la composition sociologique d'un campement à la pérennisation culturelle du Magdalénien. Apport de l'étude des savoir-faire techniques de Verberie (Oise, France) et perspectives pour le Sud-Ouest français. In: L'apprenti préhistorique : appréhender l'apprentissage, les savoir-faire et l'expertise à travers les productions techniques des sociétés préhistoriques (Klaric L., Ed.). Dolni Vestonice Studies, Brno: p. 221-244. (in French) ("From the social composition of a campsite to Magdalenian cultural reproduction: Studying technical expertise at Verberie (Oise, France) to better understand sites in the Aquitaine Basin”)

Leroyer, M. 2016, Paléontologie acheuléenne : de la technologie bifaciale à l'organisation de la subsistance collective. Etude du site de Boxgrove-Eartham (West-Sussex, Angleterre) et deux sites du cours moyen de la Seine. Thèse Paris I Panthéon Sorbonne, Paris, 619 p. (in French) (“Acheulian Paleontology: from Bifacial Technology to Collective Subsistence Organisation. Study ofBoxgrove-Eartham site (Sussex de l'Ouest, Angleterre”)

Leroyer, M. sous presse, La reconnaissance des niveaux de savoir-faire au Paléolithique ancien : le cas des maîtres et apprentis tailleurs de biface de Boxgrove (Angleterre), il y a 500000 ans. In: L'apprenti préhistorique : appréhender l'apprentissage, les savoirfaire et l'expertise à travers les productions techniques des sociétés préhistoriques (Klaric L., Ed.). Dolni Vestonice Studies, Brno: p. 10-35. (in French) (“Identifying different skill levels in the Lower Palaeolithic: Master and apprentice biface knappers at Boxgrove (England)”)

Large, D., Müller, K. \& Reiche, I. 2011, Approche analytique pour l'étude des ivoires archéologiques. Les défenses d'éléphant du site de Jinsha (1200-650 BC, Sichuan, Chine). ArchéoSciences, 35: 167-177. (in French) (“Analytic Approach for Study of Archeological Ivories. The Elephant's Tusks from Jinsha site (1200-650 BC, Sichuan, China”) doi:10.4000/archeosciences.3103

Le Dosseur, G. 2006, La Néolithisation au Levant Sud à travers l'exploitation des matières osseuses. Etude techno-économique de onze séries d'industries osseuses du Natoufien au PPNB récent, Thèse de Doctorat, Paris I Panthéon Sorbonne, Paris, 884 p. (in French) ("Neolithisation in South Levant throw the Exploitation of Bone Materials. Techno-economic Study of Eleven Series of Bone Industries from Natoufian to Recent PPNB”)

Le Dosseur, G. 2014, The production of rods in the Levant: Variability of the Methods Used during the Neolithization. In: Prehistoric Exploitation of hard animal materials, An overview of the Exploitation of hard animal materials during the Neolithic and Chalcolithic/O privire asupra exploatării materiilor dure animale de-a lungul Neoliticului și Calcoliticului (Margarit M., Le Dosseur G. \& Averbouh A., Eds.), Proceedings of the GDRE PREHISTOS Work-Session in Târgoviste, Romania, november 2013, Bilingual English-Romanian, Editura Cetatea de Scaun, Târgoviste: p. 19-42. (en anglais et en Roumain) ("La production de baguettes au Levant: variabilité des méthodes utilisées pendant la néolithisation”)

Leeuw, S. van der \& Torrence, R. 1989, What's new? A closer look at the process of innovation, Unwin Hyman, London, 353 p. (en anglais) ("Quoi de neuf ? un regard plus pointu sur le processus d'innovation”) 
Leeuw, S. van der 1994, Innovation et tradition chez les potiers mexicains ou comment les gestes techniques traduisent les dynamiques d'une société. In: Intelligence Sociale des Techniques. Des Babouins aux missiles de croisière (Latour, B. \& Lemonnier, P., Eds.), Editions La Découverte, Paris: 310-328. (in French) ("Innovation and Tradition among the Mexican Potters or how Technical Gestures send back the Dynamics of a Society”)

Legrand-Pineau, A. \& Sidéra, I. 2014, Looking for New Methodologies for Studying Bone, Antler and Tooth Industry. First Setting of Image Analysis. Proceedings of the 9 th meeting of the (ICAZ) Worked Bone Research Group, Zhengzhou, China, 2013, Zooarchaeology, Vol. 2, Henan Provincial Institute of cultural Relics and Archaeology. Culture Relics Press. academia edu, Zhengzhou: 63-67; (en anglais) (“A la recherche de nouvelles méthodologies pour l'étude de l'industrie en os, en bois et en dent. Première mise en place d'une analyse d'image”)

Legoupil, D. \& Pigeot, N. 2009, Les grandes pointes foliacées du type «Ponsonby »o: un traceur culturel en Patagonie australe. Journal de la société des américanistes, 95(2): 732. (in French) (“The Large "Ponsonby” Leafy Tips: a Cultural Tracer in Austral Patagonia”) doi:10.4000/jsa.10941

Lemonnier, P. 1976, La description des chaînes opératoire - Contribution à l'analyse des systèmes techniques. Techniques \& Culture, Bulletin de l'Équipe de recherche 191(1): 100-151. (in French) ("The Chaînes Opératoires Description: Contribution to Technical Systems Analysis”)

Lemonnier, P. 1980., Les salines de l'Ouest. Logique technique logique sociale. Editions de la MSH, Presses Universitaires de Lille, Paris: 222 p. (in French) (“The West Salt-Pans. Technical Logic Social Logic”)

Lemonnier, P. 1984, Paludiers de Guérande. Production du sel et histoire économique. Institut d'ethnologie, Paris: 282 p. (in French) ("Salt Workers of Guerande. Salt Production and Economic History")

Lemonnier, P. 1986, The Study of Matérial Culture Today: Towards an Anthropology of Technical Systems. Journal of Anthropological Archaeology, 5: 147-186. (en anglais) ("L'étude de la culture matérielle aujourd'hui : vers une anthropologie des systèmes techniques”) doi:10.1016/0278-4165(86)90012-7

Lemonnier, P. 1992, Elements for an Anthropology of Technology. University of Michigan Museum of Anthropology, Anthropological paper Vol. 88,Ann Arbor: 129 p. (en anglais) ("Éléments pour une anthropologie de la technologie")

Leroi-Gourhan, A. 1952. Homo faber, Homo sapiens. Revue de synthèse, 30: 79-102. (in French) ("Homo Faber, Home Sapiens")

Leroi-Gourhan, A. 1964, Le geste et la parole. Technique et langage. Albin Michel, Paris, 323 p. (in French) (“The Gesture and the Speach; Technic and Language”)

Leroi-Gourhan, A. 1965, Le geste et la parole. La Mémoire et les rythmes. Albin Michel, Paris: 285 p. (in French) ("The Gesture and the Speach. Memory and Rhythms”)

Leroi-Gourhan, A. \& Brézillon, M. 1966, L’habitation magdalénienne $n^{\circ} 1$ de Pincevent près Montereau (Seine-et-Marne). Galla préhistoire, 9(2): 263-385. (in French) (“The $n^{\circ} 1$ Magdalenian Dwelling at Pincevent near Montereau (Seine-et-Marne)”) doi:10.3406/galip.1966.1264

Leroi-Gourhan, A. 1972, Fouilles de Pincevent. Essai d'analyse ethnographique d'un habitat magdalénien (La section 36). VIIe supplément à Gallia-Préhistoire, Editions Centre 
national de la recherche scientifique (CNRS), Paris: 334 p. et 1 vol. de plans. (in French) ("Excavations at Pincevent: Ethnographic Analysis Essay of a Magdalenian Dwelling (the 36 section)”)

Lourdeau A. 2012, The Itaparica techno-complex: the first conspicuous settlement of central and northeastern Brazil from a technological perspective. Current Research in the Pleistocene, Special edition: Southbound: 53-56. (en anglais) ("Le techno-complexe d’Itaparica : le premier campement remarquable du centre et du nord-est du Brésil d'un point de vue technologique”)

Lourdeau, A, Hoeltz, S. \& Viana, S. 2014, Early Holocene Blade Technology in Southern Brazil. Journal of Anthropological Archaeology, 35: 190-201. (en anglais)

(“Technologie laminaire à l’Holocène ancien au sud Brésil”) doi:10.1016/j.jaa.2014.06.003

Lycett, S. \& Chauhan P. 2010, New Perspectives on Old Stones. Analytical Approaches to Paleolithic Technologies. Springer Science \& Business Media, 345 p. (en anglais) ("Nouvelles perspectives sur les pierres anciennes. Approche analytique des technologies paléolithiques”) doi:10.1007/978-1-4419-6861-6

Maget, M. 1953, Guide d'étude directe de comportements culturels (2 ${ }^{\mathrm{ème}}$ edition, 1962), Centre national de la recherche scientifique (CNRS), Paris, 268 p. (in French) ("Guide for Direct Study of Cultural Behaviours")

Manca, L. 2013, Fonctionnement des sociétés de la fin du Néolithique au début de l'Age du cuivre en Sardaigne. Une approche inédite à partir de l'étude des productions en matières dures animales. Thèse de doctorat, Université de Provence, Aix-en-Provence, 764 p. (in French) ("Functioning of Societies from the End of Neolithic to the beginning of Copper Age in Sardinia. An Original Approach from the Study of Hard Animal Materials Productions")

Manca, L. 2016, The method of débitage by Bipartition in the Exploitation of Bone. An Overview of its Application in Neolithicgroups of Sardinia. Quaternary International, 450: 224-242. (en anglais) ("La méthode de débitage par partition dans l'exploitation de l’os. Un aperçu de ses applications par les groupes néolithiques de Sardaigne”) doi:10.1016/j.quaint.2016.09.027

Manem, S. 2010, Des habitats aux sites de rassemblement à vocation rituelle : l'âge du Bronze selon le concept de «chaîne opératoire ». Les Nouvelles de l'Archéologie, 119: 30-36. (in French) ("From Dwellings to Assembling Sites with Ritual Vocation: Bronze Age through the Chaîne Operatoire Concept”) doi:10.4000/nda.965

Marquebielle, B. 2014, Le travail des matières osseuses au Mésolithique. Caractérisation technique et économique à partir de séries du sud et de l'est de la France. Thèse de Doctorat, Université de Toulouse2-Le Mirail, Toulouse: 510 p. (in French) ("The Bone Materials Working during Mesolithic. Technical and Economical Characterization from Series of South and East of France”)

Mauss, M. 1947, Manuel d'ethnographie. Payot (réédition 1967), Paris: 264 p. (in French) ("Manual of Ethnography”) doi:10.1522/cla.mam.man

Müller, K. \& Reiche, I. 2011, Differentiation of Archaeological Ivory and Bone Materials by Micro-PIXE/PIGE with Emphasis on two Upper Palaeolithic Key Sites: Abri Pataud and Isturitz, France. Journal of Archaeological Science 38: 3234-3243. (en anglais) ("Différenciation de matériaux archéologiques en ivoire et en os par MicrPIXE:PIGE en 
mettant l'accent sur deux sites clés du Paléolithique supérieur : Abri Pataud et Isturitz, France”) doi:10.1016/j.jas.2011.06.029

Noret, J. 2012, Redistributions du travail funeraire et transformations de la chaîne opératoire du cadavre dans le Bénin méridional. In: Rencontre autour du cadavre (Guy, H., JeanJean, A, Richier, A., Schmitt, A., Sénépart, I. \& Weydert N., Eds.), Marseille, GAAF: 99-108. (in French) ("Redistribution of the Funerary Work and Chaîne Operatoire of the Corpse Transformation in the Southern Benin”)

Notae Praehistoricae (Eds.) 1981, Notae Praehistoricae, Vol. 1. Collectif, Liège. (in French) URL: http://www.naturalsciences.be/mars/groups/fnrs-contact-group/notaepraehistoricae/notae-praehistoricae-1-1981

Notae Praehistoricae (Eds.) 1983, Notae Praehistoricae, Vol. 3. Collectif, Liège. (in French) URL: http://www.naturalsciences.be/mars/groups/fnrs-contact-group/notaepraehistoricae/notae-praehistoricae-1-1981

Notae Praehistoricae (Eds.) 1985 Notae Praehistoricae, Vol. 5. Collectif, Liège. (in French) URL: http://www.naturalsciences.be/mars/groups/fnrs-contact-group/notaepraehistoricae/notae-praehistoricae-5-1985

Noten, F.L. van, 1978, Les chasseurs de Meer. 2 vol., Brugge, De tempel, Dissertationes archaeologicae gandenses 18: 172 p. (in French) (“the Hunters of Meer”)

Olive, M. 1985, En marge des unités d'habitation d'Étiolles : les foyers d’activité satellites. Gallia Préhistoire, 34(1): 85-140. (in French) ("Margins of Dwelling Units of Etiolles: the satellite hearths of activity”) doi:10.3406/galip.1992.2298

Olive, M. 1988, Une Habitation Magdalénienne d'Etiolles. L'Unité P 15. Mémoires de la Société Préhistorique Française, Tome XX, Société Préhistorique Française, Paris, 174 p. (in French) (“A Magdalenian Dwelling at Etiolles. The P 15 Unit”)

Orliac, M. 1975, Empreintes au latex des coupes du gisement magdalénien de Pincevent: Technique et premiers résultats. Bulletin de la Société Préhistorique Française, 72(9): 274-276. (in French) ("Latex Prints of Sections from the Magdalenian Site of Pincevent: Technic and First Results”) doi:10.3406/bspf.1975.8392

Orliac, M. 1989, La stratigraphie des limons de Pincevent. Le Temps de la Préhistoire, 23è Congrès de la SPF, Paris: 132-133. (in French) (“Stratigraphy of Pincevent silts”)

Pelegrin, J. 1984, Rapport de recherche expérimentale au centre de Lejre (Danemark). Nanterre, Maison de l'Archéologie et de l'Ethnologie René Ginouvès, UMR 7055, 1986. (in French) ("Report of Experimental Research at Lejre Center (Denmark)") doi:10.5169/seals-169603

Pelegrin, J. 1985, Reflexions sur le comportement technique. In: La signification culturelle des industries lithiques, Actes du colloque de Liège 3-7 octobre 1984 (Otte, M. Ed.), Studia Praehistorica Belgica Vol. 3, BAR International Series Vol. 239, Hadrian Books, Oxford: p. 72-91 (in French) ("Reflection about technical behavior")

Pelegrin, J. \& Karlin, C. 1988, Entrées : Chaîne opératoire, Remontage, Taille, Technologie. In: Dictionnaire de la Préhistoire (Leroi-Gourhan, A., Ed.), Puf. (in French) (“Entrances: Chaîne Operatoire, Refitting, Knapping, Technology”)

Pelegrin, J., Karlin, C. \& Bodu, P. 1988, Les chaînes opératoires : un outil pour le préhistorien. In: Technologie préhistorique, Notes et monographies techniques, $\mathrm{n}^{\circ} 25$, 
ed. C.N.R.S., Vallebonne: 55-62. (in French) (“Chaînes Operatoires: a Tool for the Prehistorian”)

Pelegrin, J. 1991a, Aspects de démarche expérimentale en technologie lithique. In: 25 ans d'études technologiques en préhistoire, actes des XIè Rencontres Internationales d'Archéologie et d'Histoire , 18-20 octobre 1990, Editions Association pour la promotion et la diffusion des connaissances archéologiques (APDCA), Antibes: 57-64. (in French) (“Aspects of Experimental Approach in Lithic Technology”)

Pelegrin, J., 1991b, Les savoir-faire : une très longue histoire. Terrain n 16: 106-113. (in French) ("The Know-How: a Very Long Story") doi:10.4000/terrain.3001

Pelegrin, J. 1993, A Framework for Analysing Prehistoric Stone Tools Manufacture and a Tentative. Application to Some Early Lithics Industries. In: The Use of Tools by Human and Non-human Primates (Berthelet, A. \& Chavaillon, J., Eds.), Oxford University Press, Oxford: p. 302-314. (en anglais) ("Une trame pour analyser la fabrication des outils en pierre préhistoriques et une tentative. Application à quelques industries lithiques anciennes”) doi:10.1093/acprof:oso/9780198522638.003.0018

Pelegrin, J. [1986] 1995a, Technologie lithique : le Châtelperronien de Roc de Combe (Lot) et de La Côte (Dordogne). Cahiers du Quaternaire, 20, Centre national de la recherche scientifique (CNRS), Paris, 297 p. (in French) ("Lithic Technology: the Châteperronian of Roc de Combe (Lot) and of La Côte (Dordogne)”)

Pelegrin, J. [1986] 1995b, Technologie lithique : une méthode appliquée à l'étude de deux séries du Périgordien ancien (Roc-de-Combe, couche 8 - La Côte, niveau III). Thèse de Doctorat de l'Université de Paris X, Texte multigraphié, Paris, 584 p. (in French) ("Lithic Technology: a Method Applied to the Study of Two Ancient Perigordian Series (Roc-de-Combe, Level 8 - La Côte, Level III)”)

Pelegrin, J. 2000, Les techniques de débitage laminaire au Tardiglaciaire : critères de diagnose et quelques réflexions. In: L'Europe centrale et septentrionale au Tardiglaciaire, Table-Ronde de Nemours, 13-16 mai 1997 (Valentin, B., Bodu, P. \& Christensen, M., Eds.), Mémoires du Musée de Préhistoire d’Ile-de-France, Nemours, APRAIF: 73-86. (in French) (“The Blade Knapping Technics during the Late Glacial: Criteria for Diagnosis and some reflections”)

Pelegrin, J. 2004, Sur le milieu intérieur d'André Leroi-Gourhan : Eléments pour l'analyse de la taille des outils en pierre et autres réflexions. In: Autour de l'Homme, Contexte et Actualité d'André Leroi-Gourhan (Audouze, F. \& Schlanger, N., Eds.), Antibes, éditions Association pour la promotion et la diffusion des connaissances archéologiques (APDCA): 149-162. (in French) (“Toward the Internal Environment of André LeroiGourhan: Element for Stone Tools Analysis and other Considerations”)

Pelegrin, J. 2005, Remarks about Archaeological Techniques and Methods of Knapping: Elements of a Cognitive Approach to Stone Knapping. In: Stone Knapping: the necessary condition for a uniquely hominid behaviour (Roux, V. \& Bril, B., Eds.), Mac Donald Institute monograph series, Cambridge, Mac Donald Institute: 23-33. (en anglais) ("Remarques sur les techniques et les méthodes de débitage archéologique : élements pour une approche cognitive du débitage de la pierre”)

Pelegrin, J. 2007, Réflexions sur la notion de spécialiste dans la taille de la pierre au Paléolithique [ Extrait]. In: Arts et cultures de la Préhistoire. Hommages à Henri Delporte, Paris, Comité des Travaux Historiques et Scientifiques: 315-318. (in French) ("Reflections about the Notion of Expert in Knapping Stone during Paleolithic") 
Pelegrin, J. \& Yamanaka, I. 2007, From the Smallest to the Largest: Experimental Flaking by Pressure Techniques. French original text: Débitage par pression : du plus petit au plus grand. 1988, revised and enlarged from new results (in Japanese + English summary), Cultura Antiqua, 58(4): 116. (en anglais et French with summary in Japanese and English) (“Du plus petit au plus grand : extraction par les techniques de pression”)

Pelegrin, J. 2009, Sur les débitages laminaires du Paléolithique supérieur. In: François Bordes et la Préhistoire (Delpech, F. \& Jaubert, J., Eds.), 134e Congrès national des sociétés historiques et scientifiques, Documents préhistoriques, Editions du Comité des travaux historiques et scientifiques (CTHS), Bordeaux: 141-150. (in French) ("Upon the Upper Paleolithic Blades Debitage”)

Pelegrin, J. 2010, Les techniques de débitage laminaire au Tardiglaciaire : critères de diagnose et quelques réflexions. Arkeotek, 4, c1. (in French) ("The Blade Knapping Technics during the Late Glacial: Criteria for Diagnosis and some reflections”)

Pelegrin, J. 2012, New Experimental Observations for the Characterization of Pressure Blade Production Techniques. In: The Emergence of Pressure Knapping: From Origin to Modern Experimentation (Desrosiers, P., Ed.), New York, Springers Edition: 465-500. (en anglais) ("Nouvelles observations experimentales de la caractérisation des techniques de production laminaire par pression”) doi:10.1007/978-1-4614-2003-3_18

Pelegrin, J. \& Yamanaka, I. 2016, The Yokochimi Japanese Upper Palaeolithic Collection viewed from a French Lithic Technology Eye, Cultura Antiqua, 167: 64-94. (en anglais) ("La collection japonaise Paleolithique supérieur de Yokochimi vue par un oeil formé à la technologie lithique française")

Perlès, C. 1981, Economie du débitage et économie des matières premières : deux exemples grecs. Préhistoire et Technologie lithique, Cahier n 1 de l’URA $28 \mathrm{n}^{\circ}$ 1: 37-41. (in French) (“Knapping Economy and Economy of Raw Materials: Two Greek Exemples”)

Perlès, C.1987, Les industries lithiques taillées de Franchthi. Tome I : Présentation générale et industries paléolithiques. Indiana University Press, Bloomington-Indianapolis: 355 p. (in French) ("The Lithic Knapped Industries of Franchthi. Volume 1: General Presentation and Paleolithic Industries”)

Perlès, C. 1991a, Les industries lithiques taillées de Franchthi. Tome II : Les industries du Mésolithique et du Néolithique initial. Avec des contributions de Aspinal, A., Renfrew, C. \& Vaughan, P., Indiana University Press, Bloomington-Indianapolis: 288 p. (in French) ("The Knapped Lithic Industries of Franchthi. Volume II: The Mesolithic and Early Neolithic Industries”)

Perlès, C. 1991b, Économie des matières premières et économie du débitage: deux conceptions opposées ? In: 25 ans d'études technologiques en Préhistoire: bilan et perspectives. Actes des rencontres 18-19-20 oct. 1990, Editions Association pour la promotion et la diffusion des connaissances archéologiques (APDCA), Juan-les-pins: 35-45. (in French) ("Raw Materials Economy and Knapping Economy: Two Opposed Conceptions?”)

Perlès, C. 2004, Les industries lithiques taillées de Franchthi (Argolide, Grèce) 3, du Néolithique ancien au Néolithique final. Vol. 3, Excavation at Franchti Cave, 11, Indiana University Press, Bloomington: 336 p. (in French) ("The Lithic Knapped Industries of Franchthi (Argolide, Greece) 3, From Early Neolithic to Recent Neolithic") 
Perlès, C. 2016, La technologie lithique de part et d'autre de l'Atlantique. Bulletin de la Société Préhistorique Française, 113(2): 221-240. (in French) (“Lithic Technology on Both Sides of Atlantic")

Pétillon, J.-M., Bignon, O., Bodu, P., Cattelain, P., Debout, G., Langlais, M., Laroulandie, V., Plisson, H. \& Valentin, B. 2011, Hard Core and Cutting Edge: Experimental Manufacture and Use of Magdalenian Composite Projectile Tips. Journal of Archaeological Science, 38(6): 1266-1283. (En anglais) (“Noyau dur et bord tranchant : fabrication et utilisation expérimentale de projectile composite magdalénien”) doi:10.1016/j.jas.2011.01.002

Pigeot, N. 1983, Les Magdaléniens de l'unité U5 d'Etiolles : étude technique, économique, sociales de la dynamique du débitage. Thèse de 3e cycle, Université Paris 1, Paris, 3 vol., Paris, 401 p. (in French) ("The Magdalenians from Etiolles Unit U5: Technical, Economical and Social Study of the Knapping Dynamic”)

Pigeot, N.1986, Apprendre à débiter des lames : un cas d'éducation technique chez les Magdaléniens d’Étiolles. Bulletin de la Société Préhistorique Française, 83: 67-69. (in French) ("To learn how to knappe blades: a case of technical training among the Magdalenians of Etiolles”)

Pigeot, N. [1983 Thèse] 1987, Magdaléniens d'Etiolles : économie de débitage et organisation sociale. $\mathrm{XXV}^{\circ}$ supplément à Gallia Préhistoire. Centre national de la recherche scientifique (CNRS), Paris: 10-22. (in French) ("The Magdalenians from Etiolles: Knapping Economy and Social Organisation”)

Pigeot, N. 1990, Technical and Social Actors: Flintknapping Specialists and Apprentices at Magdalenian Étiolles. Archaeological Review from Cambridge, 9(1): 126-141. (En anglais) (“Acteurs techniques et sociaux : spécialistes du débitage et apprentis tailleurs au Magdalénien à Etiolles”)

Pigeot, N. 1991, Réflexions sur l'histoire technique de l'Homme : de l'évolution cognitive à l'évolution culturelle. Paléo, 3(1): 167-200. (in French) ("Reflexions about Technical History of Man: from Cognitive Evolution to Cultural Evolution”) doi:10.3406/pal.1991.1046

Pigeot, N. (Ed.), 2004, Les derniers Magdaléniens d'Etiolles : perspectives culturelles et paléohistoriques, l'unité d'habitation Q31. Suppl. Gallia Préhistoire, 37, Paris, Centre national de la recherche scientifique (CNRS): 351 p. (in French) (“The last Magdalenians of Etiolles: Cultural and Paleohistoric Perspectives, the Dwelling Unit Q31”)

Pigeot, N. \& Philippe, M. 2004, Bases documentaires et méthodologiques. In: Les derniers magdaléniens d'Etiolles : perspectives culturelles et paléohistoriques, l'unité d'habitation Q31 (Pigeot, N., Ed.), 2, Paris, Centre national de la recherche scientifique (CNRS), Suppl. Gallia Préhistoire, 37: 31-39. (in French) (“Documentary and Methodologic Bases”)

Pigeot, N. 2011, Chaînes opératoires : contexte théorique et potentiel cognitif. In: L’archéologie cognitive (Treuil, R., Ed.), Cogniprisme, Paris, Editions de la Maison des Sciences de l'Homme: 149-171. (in French) ("Chaînes Operatoires: Theoretical Background and Cognitive Potential”) 
Ploux, S. 1979-1980, Proposition pour l'étude du débitage expérimental. Maîtrise de 2e cycle, Paris X Nanterre, Paris, 157 p. (in French) ("Proposel for the Study of Experimental Debitage”)

Ploux, S. 1989, Approche archéologique de la variabilité des comportements techniques individuels : l'exemple de quelques tailleurs de Pincevent. Thèse de doctorat, Nanterre, Université Paris X-Nanterre, Paris, 584 p. (in french) ("Archeological Approach of the Variability of Individual Tehnic Behaviour: the Exemple of Some Knappers from Pincevent”) URL: http://tel.archives-ouvertes.fr/tel-00761111

Ploux, S. 1991, Technologie, technicité, techniciens : méthodes de détermination d'auteurs et comportements techniques individuels. In: Vingt-Cinq ans d'études technologiques en préhistoire : bilan et perspectives: 201-214. (in French) (“Technology, Technicity, Technician: Method for Authors Determination and Individual Technic Behaviours”)

Ploux, S., Karlin, C. \& Bodu, P. 1992, D’une chaîne l'autre, normes et variations dans le débitage laminaire magdalénien. Techniques \& Culture, 17-18: 81-114. (in French) ("From One Chaîne to an Other, Norms and Variations in the Magdalenian Blade Knapping”) doi:10.4000/tc.687

Ploux, S., \& Karlin, C. 1994, Le travail de la pierre au Paléolithique ou comment retrouver l'acteur technique et social grâce aux vestiges archéologiques. In: De la Préhistoire aux missiles balistiques : l'intelligence sociale des techniques (Latour, B. \& Lemonnier, P., Eds.), Paris, La Découverte: 46-65. (in French) ("Stone Knapping during Paleolithic or How to Find the Technic and Social Actor thanks to the Archeological Remains")

Ploux, S. \& Karlin, C. 2014, La gestion d'un savoir-faire : le débitage. In: Un automne à Pincevent. Le campement magdalénien du niveau IV20 (Julien, M. \& Karlin, C., Eds.), SPF mémoire 57. Paris: 113-134. (in French) (“A Know-How Management: the Debitage”)

Ploux, S., Karlin, C., Julien, M., Enloe, J. G. \& Hardy, M. 2014, L'Unité de résidence $27-$ M89. In: Un automne à Pincevent. Le campement magdalénien du niveau IV20 (Julien, M. \& Karlin, C., Eds.), SPF mémoire 57. Paris: 211-265. (in French) ("The Dwelling Unit 27-M89”) URL: http://www.prehistoire.org/shop_515-31884-0-0/m57-2014-unautomne-a-pincevent-le-campement-magdalenien-du-niveau-iv20-m.-julien-et-c.-karlinbr-collectif.html

Reiche, I., Chadefaux, C., Müller, K. \& Gourrier, A. 2011, Towards a Better Understanding of Alteration Phenomena of Archaeological Bone by a Closer Look at the OrganicMineral Association at Micro- and Nanoscale. Preliminary Results on Neolithic Samples from Chalain Lake Site 19, Jura, France. ArchéoSciences, Revue d'Archéométrie, Varia - international ArBoCo Workshop: 143-158. (en anglais) ("Vers une meilleur compréhension du phénomène d'altération de l'os archéologique par une observation approfondie de l'association organique et minéral a une macro et nanoéchelle. Résultats préliminaires sur des exemples néolithiques du site 19 du Lac Châlain, Jura, France”) doi:10.4000/archeosciences.3075

Rigaud, S. 2011, La parure : traceur de la géographie culturelle et des dynamiques de peuplement au passage Mésolithique-Néolithique en Europe. Thèse d'université, Université Sciences et Technologies - Bordeaux I, Bordeaux, 470 p. (in French) ("The Ornament: Tracer of Cultural Geography and Dynamic Peopling during the Transition Mesolithic-Neolithic in Europe”) 
Roussel, M., Bourguignon, L. \& Soressi, M. 2009, Identification par l'expérimentation de la percussion de calcaire au Paéolithique Moyen : le cas du façonnage des racloirs bifaciaux Qina de Chez-Pinaud, Jonzac, Charente-maritime. Bulletin de la Société Préhistorique Française, 106(2): 219-238. (in French) (“Identification by Experimentation of Calcareous Percussion during Middle Paleolithic: the Case of Shaping Quina Bifacial-Scraper from Chez-Pinaud, Jonzac, Charente-maritime”) doi:10.3406/bspf.2009.13846

Roux, V., \& Corbetta, D. 1990, Le Tour du potier. Spécialisation artisanale et compétences techniques. Éditions Centre national de la recherche scientifique (CNRS), Monographie du Centre de Recherches Archéologiques, 4, Paris-Valbonne: 168 p. (in French) ("Potter's Wheel. Crafted Specialization and Technic Competences")

Roux, V. 1991, Peux-t-on interpréter les activités préhistoriques en termes de durée d'apprentissage ? Apport de l'ethnologie et de la psychologie aux études technologiques. In: 25 ans d'études technologiques en préhistoire, Actes des XIè Rencontres Internationales d'Archéologie et d'Histoire d'Antibes, Antibes, 18-20 octobre 1990, Editions Association pour la promotion et la diffusion des connaissances archéologiques (APDCA), Antibes: 47-56. (in French) (“Can We Interpret Prehistoric Activities in Terms of Apprenticeship Duration? Contribution of Etnology and Psychology to Technologic Studies”)

Roux, V. 1997, Cognition et archéologie : Habileté impliquée dans les techniques du passé. Journal des Anthropologues, 70(1): 51-62. (in French) ("Cognition and Archeology. Ability Involved in the Past Technics”) doi:10.3406/jda.1997.2046

Roux, V. \& Matarasso, P. 1999, Crafts and the Evolution of Complex Societies: New Methodologies for Modeling the Organization of Production, a Harappan Example. In: The social dynamics of technology, practice, politics and world views (Dobres, M.-A. \& Hoffman, C., Eds.), Smithsonian Institution Press, Washington and London: 46-70. (en anglais) (“Artisanats et évolution des sociétés complexes : nouvelles méthodologies pour modéliser l'organisation de la production, un exemple Harapéen”)

Roux, V. \& Bril, B. 2002, Des programmes d'apprentissage comparables pour des actions techniques différentes. In: Le geste technique. Réflexions méthodologiques et anthropologiques (Bril, B. \& et Roux, V., Eds.), Editions Erès, Revue d’Anthropologie des connaissance, Technologies - Idéologies - Pratiques, Ramonville Saint-Agne: 231242. (in French) (“Comparable learning programs for various technical actions")

Roux, V. \& David, E. 2005, Planning Abilities as a Dynamic Perceptual-Motor Skill: an Actualist Study of Different Levels of Expertise Involved in Stone Knapping. In: Stone Knapping: the Necessary Conditions for a Uniquely Hominin Behaviour (Roux, V. \& Bril, B., Eds.), Actes du workshop de Pont-à-Mousson, 21-24 novembre 2001.

McDonald Institute Monograph Series, Cambridge: 91-108. (en anglais) (“Capacités de planification comme moteur dynamique : une étude actualiste des différents niveaux d'expertise mise en œuvre dans le débitage de la pierre”)

Roux, V. \& Bril, B., Eds. 2005, Stone Knapping: the Necessary Conditions for a Uniquely Hominin Behavior. McDonald Press. (paru en février 2006), Cambridge, 356 p. (en anglais) ("La taille de la pierre : Les conditions nécessaires pour un comportement propre aux hominidés”)

Roux, V. 2011, Habiletés et inventions : Le comportement "intelligent", un facteur aléatoire dans l'évolution des techniques. In: L'archéologie cognitive (Treuil, R., Ed.), Paris, 
Editions de la Maison des sciences de l’homme: 173-188. (in French) (“Abilities and Inventions: «Intelligent » Behaviour, a Random Factor in Technics Evolution”)

Roux, V. 2013a, Spreading of Innovative Technical Traits and Cumulative Technical Evolution: Continuity or Discontinuity? Journal of Archaeological Method and Theory, 20(2): 312-330. (en anglais) ("Developpement de traits techniques innovants et évolution technique cumulative : Continuité ou discontinuité ?”) doi:10.1007/s10816012-9153-4

Roux, V. 2013b,Mise en ligne de référentiels expérimentaux et ethnoarchéologiques sur les techniques archéologiques, Labex les passés dans le present, Université Paris Ouest Nanterre la Défense, Paris. (in French) (“On Line Experimental and Ethnoarcheological Referencials on Archeological Technics”) URL: http://passes-present.eu/fr/mise-enligne-de-referentiels-experimentaux-et-ethnoarcheologiques-sur-les-techniquesarcheologiques

Roux, V., \& Courty, M.-A. 2016, Des Céramiques et des Hommes. Décoder les assemblages archéologiques. Presses Universitaires de Paris Ouest, Nanterre: 415 p. (in French) (“Ceramics and Men. To Uncode Archeological Assemblages”)

Schlanger, N. \& Sinclair, A. Eds. 1990, Technology in the Humanities. Archaeological Review from Cambridge, Vol. 9(1), University of Cambridge, Cambridge, 186 p. (en anglais) ("La technologie dans les sciences humaines")

Schlanger, N. 1991, Le fait technique total. La raison pratique et les raisons de la pratique dans l'œuvre de Marcel Mauss. Terrain 16: 114-130. (in French) ("The Total Technical Fact. Practical Reason and Reason of Practice in Marcel Mauss works”) doi:10.4000/terrain.3003

Schlanger, N. 2004, « Suive les gestes, éclat par éclat » - la chaîne opératoire d'André LeroiGourhan. In: Autour de l'Homme. Contexte et actualité d'André Leroi-Gourhan (Audouze, A \& Schlanger, N, Eds.), Editions. Association pour la promotion et la diffusion des connaissances archéologiques (APDCA), Antibes: 127-147. (in French) ("Folowing the Gestures, Flake by Flake - Andre Leroi-Gourhan Chaîne operatoire”)

Schlanger, N. 2005, The chaîne opératoire. In: Archaeology: The Key Concepts (Renfrew, A.C. \& Bahn, P., Eds.), Routledge, London: 25-31. (en anglais) ("La chaîne opératoire”)

Schmider, B., Croisset, E. de, Boëda, E. \& Pelegrin, J. 1985, Archéologie expérimentale. Les amas litthiques de la zone N19 du gisement magdalénien de Marsangy : approche méthodologique par l’expérimentation. In: Archéologie expérimentale: 64 p. (in French) ("Experimental Archeology. The N19 Zone Lithic Concentrations of the Magdalenian site of Marsangy: Methodological Approach by Experimentation”)

Schmider, B \& Croisset, E. de 1990, The Contribution of Lithic Refittings for Spatial Analysis of Campsite H17 and D14 at Marsangy. In: The big Puzzle. International Symposium on refitting Stone Artefacts. Montrepos 1987 (Cziesla, E., Eickhoff, S., Arts, N. \& Winter, D., Eds.), Studies in Modern Archaeology Vol. 1, Holos, Bonn: 431-445. (en anglais) ("La contribution des remontages lithiques à l'analyse spatiale du campement H17 et D14 à Marsangy”)

Schmider, B. (Ed.) 1992, Marsangy : un campement des derniers chasseurs magdaléniens sur les bords de l'Yonne. ERAUL, Etudes et recherches archéologiques de l'Université de Liège $n^{\circ} 55$, Liège, 275 p. (en Français) ("Marsangy: a Settlement of the Last Magdalenians Hunters on the Yonne Riverside”) 
Simondon, G. [1958] 1989, Du mode d'existence des objets techniques. Ed. Aubier, 3e Éd. augmentée d'une préface de Hart J. et d'une postface de Deforge Y., Paris, Aubier, 333 p. (in French) (“About the Mode of Existence of Technical Objects”)

Simonet, A. 2012, Des apprentis gravettiens ont-ils confectionné des armatures lithiques à Tercis (Landes, France) ? PALEO, 23 (in French) ("Did Gravettian apprentices made lithic projectile points at Tercis (Landes, France)?”) [En ligne], 23, mis en ligne le 19 avril 2013, URL: http://journals.openedition.org/paleo/2456

Soressi, M. 2002, Le Moustérien de tradition acheuléenne du sud-ouest de la France. Discussion sur la signification du faciès à partir de l'étude comparée de quatre sites : Pech-de-l'Azé I, Le Moustier, La Rochette et la Grotte XVI. Doctorat, Thèse de l'Université Bordeaux I, Bordeaux, 330 p. (in French) ("The Mousterian of Acheulean Tradition from the South-West of France. Debate about the Facies Meaning Based on a Comparative Study of Four Sites: Pech-de-l’Azé I, Le Moustier, La Rochette and la Grotte XVI”)

Soressi, M \& Geneste, J.-M. 2011 The History and Efficacy of the Chaîne Opératoire Approach to Lithic Analysis: Studying Techniques to Reveal Past Societies in an Evolutionary Perspective. In: Reduction Sequence, chaîne opératoire, and Other Methods: The Epistemologies of Different Approaches to the Lithic Analysis, PaleoAnthropology, Special Issue (Tostevin, G.B. Ed.): 334-350. (en anglais) ("Histoire et efficacité de l'approche par la chaîne opératoire de l'analyse lithic: l'étude des technique pour restituer les sociétés du passé dans une perspective evolutioniste”) doi:10.4207/PA.2011.ART63

Stapert, D. 1990, Within the Tent or Outside? Spatial Patterns. In: Late Palaeolithic Sites, Helinium, 29(1): 14-35. (en anglais) ("Dans la tente ou en dehors ? Modèles spatiaux")

Stapert, D. 1992, Rings and Sectors: Intrasite Spatial Analysis of Stone Age Sites. Thèse de doctorat, Université de Groningen, Groningen, 236 p. (en anglais) (“Anneaux et secteurs : analyse spatiale intrasite de sites de l’Âge de la pierre”)

Stapert, D. 2007, Youngsters knapping flint near the campfire: an alternative view of site K at Maastricht-Belvédère (the Netherlands). Archäologisches Korrespondenzblatt, 37: 1935. (en anglais) ("Jeunes tailleurs de silex près du foyer : une autre vision du site $\mathrm{K}$ de Maastricht-Belvédère (les Pays-Bas)”)

Tartar, E., Teyssandier, N., Bon, F. \& Liolios, D. 2006, Equipement de chasse, équipement domestique: une distinction efficace ? Réflexion sur la notion d'investissement technique dans les industries aurignaciennes. In: Normes techniques et pratiques sociales : de la simplicité des outillages pré- et protohistoriques. (Astruc, L. Bon, F., Léa, V., Milcent, P.-Y. \& Philibert, S., Eds.), Actes du Colloque d’Antibes 2005: 107117. (in French) ("Hunting Equipment, Domestic Equipment: an Efective Distinction? Reflexion about the Notion of Technical Invest in the Aurignacian Industries”)

Tejero, J.-M. \& Christensen, M. 2011, Red Deer Antler Technology and Early Modern Humans in Southeast Europe: An Experimental Study. Journal of Archaeological Science, 39(2): 332-346. (en anglais) ("Technologie du bois de cerf et Hommes modernes anciens dans l'Europe du sud-est : une étude expérimentale”) doi:10.1016/j.jas.2011.09.018

Tixier, J. 1967, Procédés d'analyse et questions de terminologie concernant l'étude des ensembles industriels du Paléolithique récent et de l'Epipaléolithique dans l'Afrique du Nord-Ouest. In: Background to Evolution in Africa (Bishop, W. W. \& Clark, J. D., 
Eds.), Chicago University Press, Chicago: p. 771-820. (in French) (“Analysis Proceedings and Questions of Terminology about the Study of Industrial Ensembles of Upper Paleolithic and Epipaleolithic in Nord-West Africa”)

Tixier, J. (Ed.) 1980, Préhistoire et Technologie lithique. Journées du 11-13 Mai 1979 organisées par l'équipe Préhistoire et Technologie. URA 28. Centre national de la recherche scientifique (CNRS), Editions du Centre régional de publication, SophiaAntipolis, 59 p. (in French) ("Prehistory and Lithic Technology”)

Tixier, J., Inizan, M.-L. \& Roche, H. 1980, Préhistoire de la pierre taillée. T. 1 : Technologie de la Pierre taillée. C.R.E.P., Meudon, 120 p. (in French) (Prehistory of Knapped Stone:. T. 1: Technology of Knapped Stone”)

Tixier, J., Inizan, M.-L. \& Roche, H. 1984, Préhistoire de la pierre taillée. T.2 : Economie du débitage laminaire. C.R.E.P, Meudon, 166 p. (in French) ("Prehistory of Knapped Stone. T. 2: Blade Debitage Economy”)

Tixier, J. (non publié1978) 2012, Méthodes pour l'étude des outillages lithiques. A method for the study of stone tools. Notice sur les travaux de Jacques Tixier présentée en vue du grade de docteur es lettre soutenue sur travaux. Coll. Archéologiques, Luxembourg, 197 p. (in French and English; en français et en anglais)

Tostevin, G., Bleed, P., Carr, P.J., Bradbury, A.P., Shott, M. J., Lindly, J.M., Clark, G.A., Soressi, M., Geneste, J.-M., Tryon, C. \& Potts, R. 2011, Reduction Sequence, Chaîne Opératoire and Other Methods: The Epistemologies of Different Approaches to Lithic Analysis. Special Issue of PalaeoAnthropology: 293-389. (en anglais) (“ 'Reduction Sequence', chaîne opératoire et autres méthodes : Epistémologie de différentes méthodes de l'analyse du lithique”) doi:10.4207/PA.2011.ART59, SSN 1545-0031

Tostevin, G. 2011a, Introduction to the Special Issue on Reduction Sequence, Chaîne Opératoire, and other Methods: The Epistemologies of Different Approaches to lithic analysis. Special Issue of PalaeoAnthropology: 293-296. (in English) ("Introduction au numéro special :"Reduction Sequence”, chaîne opératoire et autres méthodes : Epistémologie des différentes méthodes de l’analyse du lithique”) doi:10.4207/PA.2011.ART59, SSN 1545-0031

Tostevin, G. 2011b, Levels of Theory and Social Practice in the Reduction Sequence and Chaîne Opératoire Methods of Lithic Analysis. Special Issue of PaleoAnthropology: 351-375. (in English) ("Niveaux théoriques et pratique sociale des méthodes d'analyse lithique de la "Reduction Sequence"iet de la chaîne opératoire”) doi:10.4207/PA.2011.ART64

Valentin, B. 2007, De l'Oise à la Vienne en passant par le Jourdain. HDR, Université de Paris I, Paris, vol. 1, 287 p., vol. 2: 108 p. (in French) ("From the Oise to the Vienne passing by the Jourdain”)

Valentin, B. 2008a, Jalons pour une paléohistoire des derniers chasseurs : XIVe-VIe millénaire avant J.-C. Cahiers Archéologiques de Paris I, Publications de la Sorbonne, Paris: 325 p. (in French) ("Ways for a Paleohistory of the Last Hunters: XIVe-VIe millennium before J.-C.”)

Valentin, B. 2008b, Productions lithiques magdaléniennes et aziliennes dans le Bassin parisien : disparition d'une économie programmée. The Arkeotek Journal, 2(3): 1-54. (in French) ("Magdalenian and Azilian Lithic Productions in the Paris Basin: 
Disappearance of a Programmed Economy”) URL: https://halshs.archivesouvertes.fr/halshs-00375462

Valentin, F., Rivoal, I., Thevenet, C. \& Sellier P. (Eds.) 2014. La chaîne opératoire funéraire. Ethnologie et archéologie de la mort. Maison de l'Archéologie et de l'Ethnologie, Editions de Boccard, Paris, 47 p. (in French) (The Funerary Chaîne Operatoire. Ethnology and Archeology of Death”)

Vanhaeren M. 2002, Les fonctions de la parure au paléolithique supérieur : de l'individu à l'unité culturelle. Thèse d'université, Université Sciences et Technologies - Bordeaux I, Bordeaux, 348 p. (in French) ("the Ornament Function during Upper Paleolithic: from Individual to Cultural Unity”)

Wadley, L., Murungi, M.L., Witelson, D., Bolhar, R., Bamford, M. Sievers, Ch., Val, A. \& de la Pena, P. 2016, Steenbokfontein 9KR, A Middle Stone Age Spring Site in Limpopo, South Africa. South African Archaeological Bulletin 71(204): 130-145 (en anglais) ("Steenbokfontein 9KR, un site de printemps du Paléolithique moyen a Limpopo, Afrique du Sud”) 


\title{
70 years of "Chaîne opératoire": What French prehistorians have done with it
}

\author{
Françoise Audouze, Claudine Karlin
}

UMR7041 Archéologies et Sciences de l’Antiquité. CNRS. ArScAn-Ethnologie Préhistorique, France.

Email: Audouze: francoise.audouze@mae.u-paris10.fr; Karlin: claudine.karlin@mae.u-paris10.fr

\begin{abstract}
:
This paper is about the origin and development of the French school of Prehistoric Technology. Going back to the sources, the authors show how the concept of « chaîne opératoire ", a concept proposed by André Leroi-Gourhan, who was both an anthropologist and a prehistorian, was first developed by social anthropologists, and then applied to the analysis of prehistoric lithics in strong connection with their anthropologist colleagues. The discovery of the open-air site of Pincevent (Seine-et-Marne, France) allowed Leroi-Gourhan to develop his ideas about an ethnographic approach to a prehistoric settlement. With this in mind, the refitting of flint artefacts, used to restore the débitage sequences, was systematically used for the first time. Interpreting these refits forced him to develop the chaîne opératoire concept. At the same time, Jacques Tixier adopted this tool relying on the interpretation of experimental débitage practiced by his team. Pooling concepts developed by Tixier's team and LeroiGourhan's laboratory, practicing flint knapping, experimentation and flint core refitting led to developments that were at first about knapping techniques and methods. This cooperation helped refine the recognition of skill levels, from apprenticeship to high performance, and, beyond that, to identify individual knappers inferred from idiosyncratic knapping characteristics of refitted flint blocks. This work opened the way to the economic realm and social organization inferred from spatial analysis of Upper Palaeolithic living floors. Later use of this concept and of all lines of inquiry it opened, extended to every prehistoric period (from Early Palaeolithic, Neolithic and Bronze Age), to other countries and to other areas than lithics: bone, organic materials, ceramics, metal, archaeozoology and archaeology of mortuary practices. It lead to the development of adapted concepts and criteria, and new methods and techniques of observation and experimentation adapted to these materials or practices. While based on previous advances, two recent approaches focusing on the middle and long term open new directions of research. Thus, beyond these two interconnecting currents that developed around Leroi-Gourhan and Tixier, multiple interactions led to a reciprocal appropriation of these heritages giving the "French" chaîne opératoire its characteristics:
\end{abstract}

- the interaction of ethnologists of "cultural technology" and prehistorians on this approach;

- an anthropological background implicit in the approach of prehistorians, becoming more explicit with the introduction of ethnoarchaeological data;

- rigorous and detailed empirical research at the base of the analytical process;

- backed up by reproducible experimental research;

- creation of original concepts for the production of lithic tools;

- taking into account psychomotor factors in the analysis of technical behavior;

- a posteriori models rather than a priori theoretical models;

- a diversification of applications by extension to neighboring domains.

Given its flexibility, dynamic vision of processes and structural connection from the technical sphere to all human dimensions, the chaîne opératoire is an effective mechanism for thinking about production processes and their place in society. In this article, we have tried to trace a path, to show 
the different development stages of this concept in France and to briefly report on the multiple avenues of research in which it became a stakeholder or centerpiece.

Keywords: chaîne opératoire; debitage management; raw material management; experimentation; method; mental refitting; know-how; technical system; technique 